\title{
Progress Report \\ Experimental and Theoretical Investigation of High Gradient Acceleration
}

\author{
Submitted to \\ Department of Energy \\ Washington, D.C. \\ Prepared by \\ Dr. G. Bekefi \\ Dr. C. Chen \\ Dr. S. Chen \\ Dr. B. Danly \\ Dr. R.J. Temkin \\ Dr. J. S. Wurtele \\ Plasma Fusion Center \\ Massachusetts Institute of Technology \\ Cambridge, Massachusetts 02139 \\ Duration of Research \\ June 1,1991-Feb. 1, 1992
}

DOE/ER/40648--1

DE92 015299

February, 1992

\section{DISCLAIMER}

This report was prepared as an account of work sponsored by an agency of the United States Government. Neither the United States Government nor any agency thereof, nor any of their employees, makes any warranty, express or implied, or assumes any legal liability or responsibility for the accuracy, completeness, or usefulness of any information, apparatus, priduct, or process disclosed, or represents that its use would not infringe privately owned rights. Refer. ence herein to any specific commercial product, process, or service by trade name, tridemark, manufacturer, or otherwise does not neicessarily constitute or imply its endorsement, recommendation, or favoring by the United States Government or any agency thereof. The views and opinions of authors expressed herein do not necessarily state or reflect those of the United States Government or any agency theroof.

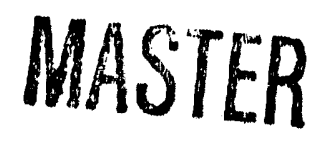


Appendix C. Cavity Design and Vacuum System

Appendix D. CARM Amplifier Status Report

Appendix E. 33GIIz FEL for Accelerator Systems

Appendix F. Selected Reprints and Preprints:Theory (Removel) -

Appendix G. Publications Supported by This Proposal 


\section{Summary}

This report contains a technical progress summary of the research conducted under the auspices of DOE Grant No. DE-FG0291ER-40648, "Experimental and Theoretical Investigations of High Gradient Acceleration." This grant [1] supports three research tasks: Task $A$ consists of the design and fabrication of a $17 \mathrm{GHz}$ rf photocathode gun, Task B supports the testing of high gradient acceleration using a $33 \mathrm{GHz}$ structure, and Task $\mathrm{C}$ comprises theoretical investagations, both in support of the experimental tasks and on critical physics issues for the development of high energy linear colliders.

This report is organized as follows. The development of an $\mathrm{rf}$ gun design and research progress on the picosecond laser system is summarized in Sec. 2, The status of the studies of the LBL/Haimson high gradient structure, using a $50 \mathrm{MW}$ freeelectron laser is summarized in Sec. 3, and theoretical research progress is described in Sec. 4. Supporting material is contained in Appendices A-G.

\section{Experimental research on $\mathbf{r f}$ photocathode guns}

\subsection{Overview}

The main goal of the $17 \mathrm{GHz}$ photocathode RF gun experiment at MIT is to study particle acceleration at high field gradients and the production of high quality beams from high frequency RF guns. Initial experiments are designed around an accelerating gradient of $\sim 250 \mathrm{MV} / \mathrm{m}$, with an RF frequency of $17.136 \mathrm{GHz}(6 \times \mathrm{SLAC})$. High frequency $R F$ acceleration requires less power than its lower frequency counterpart and may lead to compact, short-pulse, high brightness electron beams suitable for next generation linear collider applications. During the period (June 1991 to February 1992), we have made progress in the following areas: timing scheme, laser system, RF gun cavity, CARM amplifier, electron beamline, and RF gun frequency scaling. Details of these topics are described below.

\subsection{Status of the Experimental Program}

\subsubsection{Timing scheme and jitter control}

The timing scheme between the microwave system (CARM) and the laser system has been studied in detail. With the timing sequence and constraints on the jitter determined, it is then possihle to optimize the choice of the mode lock frequency of the laser oscillator, and finalize the laser parameters. Due to the long lead-time involved for the laser system, namely for component integration and testing, it is important to initiate the purchasing phase as early as possible, although the laser itself will not be employed in the Girst phase of our experimental program.

Figure 1 shows the spatial layout and the interconnections of the laser, the microwave, and the trigger systenis. A tunable two-frequency source produces the high 


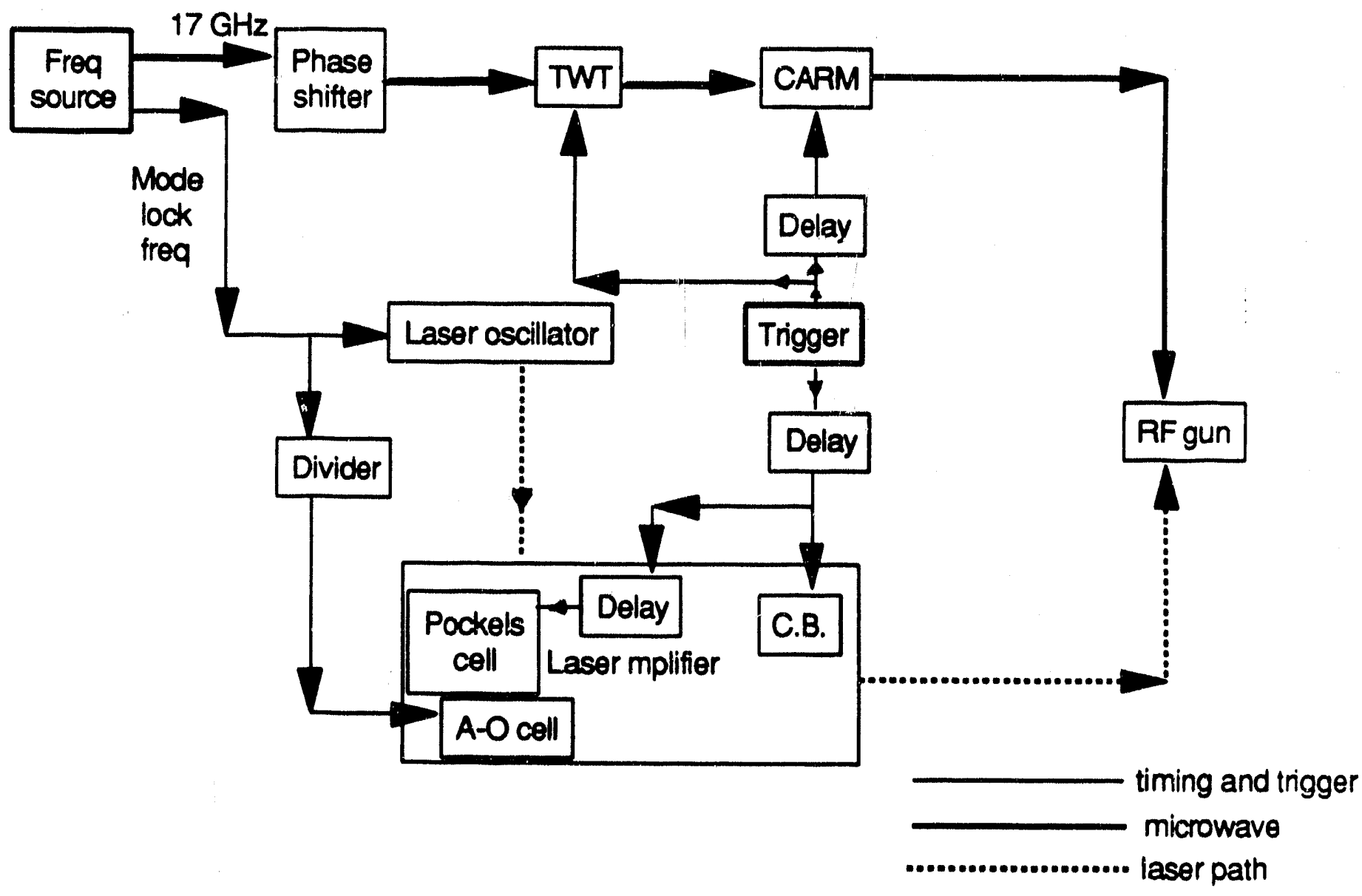

Fig.1 Interconnections between the microwave, the laser and the trigger systems 
$(17 \mathrm{GHz}$ ) and the low (mode-lock frequency of about $100 \mathrm{MHz}$ ) frequency signals required to run our experiment. The $17 \mathrm{GHz}$ arm of the source runs $\mathrm{CW}$ at the mw level. The other arm of the frequency source runs at about $100 \mathrm{MHz}$ (mode lock frequency) and drives the mode locker in the laser oscillator. The laser oscillator produces a continuous train of pulses with an inter-pulse spacing corresponding to the mode lock frequency. The laser path is denoted by the dotted line in Figure 1. At this stage, both the RF and the laser signals are CW.

The RF gun is fired by sending a trigger pulse to the microwave amplifier chain and the laser amplifier. The $17 \mathrm{GHz}$ output (at $\mathrm{mw}$ level) drives an intermediate TWT amplifier and generates $\mathrm{kW}$ output at the same frequency. Final stage amplification by the CARM amplifier brings the power level to 5-10 MW. The high power microwave is then transported to the RF gun cavity. The trigger pulse is also properly timed to trigger the laser gain build-up and the single pulse selection processes. The single amplified laser pulse is transported to the RF gun.

The timing sequence between the two arms of the experiment is shown on time axis in Figure 2. Before the arrival of the trigger pulse, the system essentially runs CW. The trigger pulse then initiates a series of events in the laser and microwave amplifier chain and the two pulses (microwave and laser) are generated. The timing system is designed to bring them to the gun cavity at the right time with the right phase relationship. Notice we used the words "time" and "phase" in describing the timing relationships. It is necessary because:

(a) There are two distinctive time scales involved. The first one is the arrival "time" of the pulses. The laser pulse should arrive after the RF cavity is filled and before the cavity field begins to decay. We named this arrival-time uncertainty the "timing jitter". The second one is the microwave phase the laser occupies when it impinges on the cathode. We named this arrival-phase uncertainty the "phase jitter". In our experiment, we require the timing jitter to be a few nanoseconds and the phase jitter to be a few picoseconds.

(b) The arrival-time and the arrival-phase are governed to a large extent by different processes in the timing chain. The arrival-time jitter is induced by the uncertainties in the amplifiers' (both CARM's and laser's) gain envelope position in time. The sources of jitter are: voltage and gain build-up time jitter, pockels cell firing jitter, and trigger delay jitter. The arrival-phase jitter is related to the oscillator's stability and to the variation of the path-length sensitive elements in the system: optical fiber, waveguide length, and optical path length. Of course, the phase shifts generated in the high power amplifiers (CARM and the laser amplifier) also contribute.

We will attempt to minimize both timing jitter and phase jitter.

The timing jitter in the CARM amplifier has been partially characterized. The arrival-time of the induction LINAC voltage pulse to the CARM electron gun is measured as a function of pulse voltage. The histograms of the arrival-time jitter for 3 voltages are shown in Figure 3. The standard deviation of the jitter is between 20 and $40 \mathrm{~ns}$ at high voltages. The timing jitter is greatly reduced if the trigger pulse is locked to the $60 \mathrm{~Hz}$ line. Figure 4 shows the improvements of this synchronization scheme. 


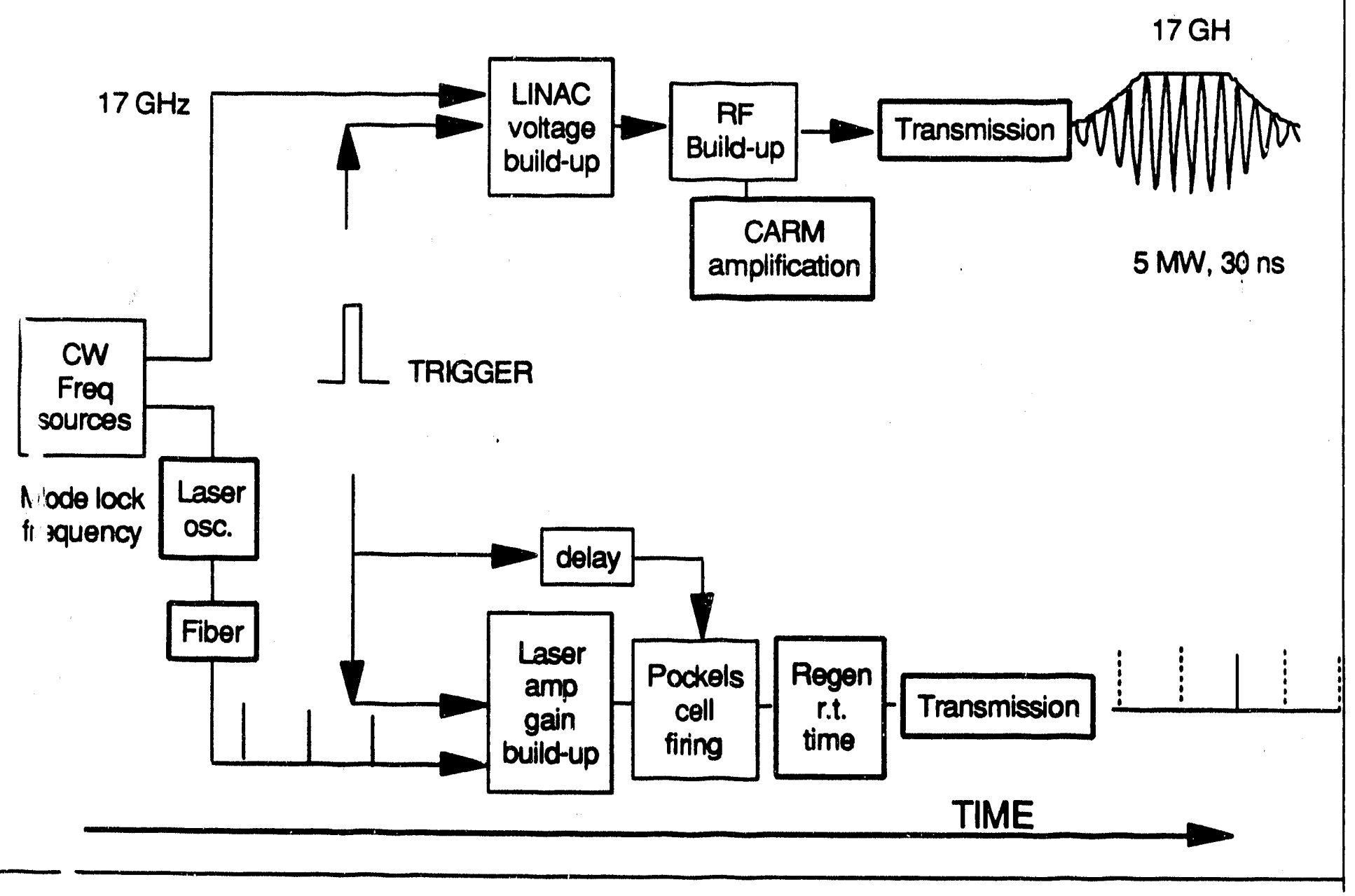

Fig.2 Timing sequence of the photocathode RF gun experiment 


\section{Linac Pulse Timing Jitter Pulse NOT synched to $60 \mathrm{~Hz}$}

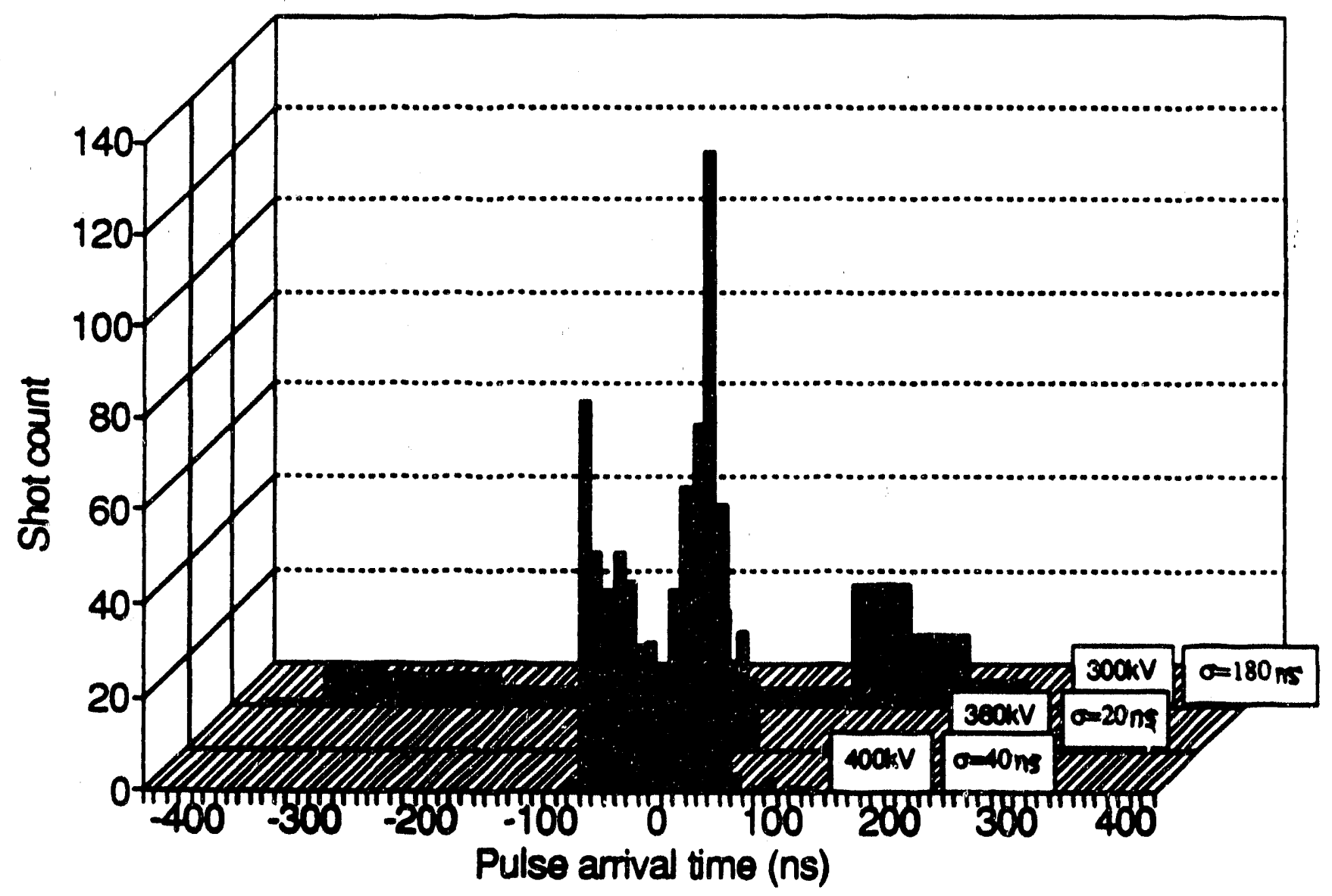

Fig.3 Histograms of the arrival-time jitter for 3 voltages. 


\section{Linac Pulse Timing Jitter Pulse synched to $60 \mathrm{~Hz}$}

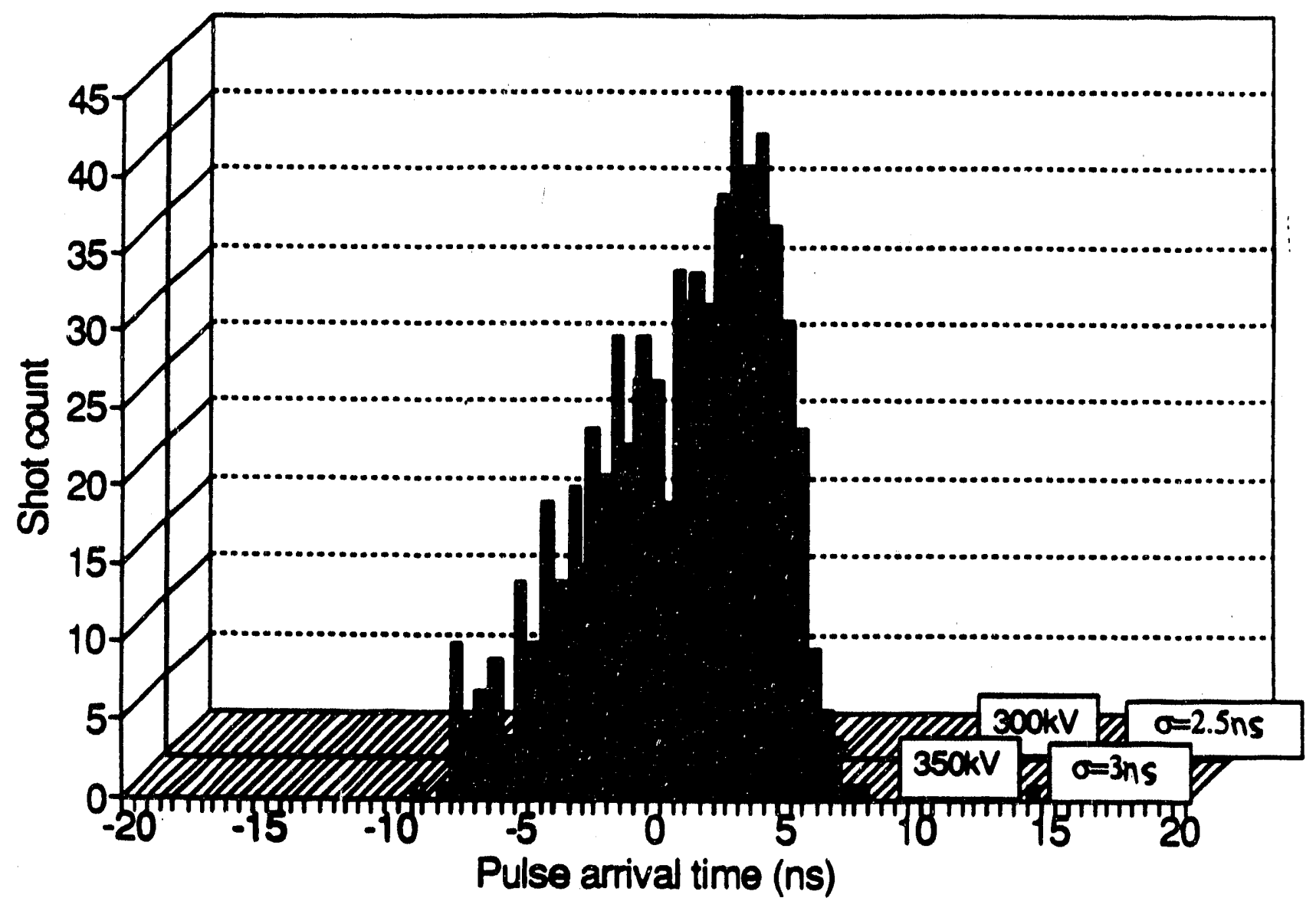

Fig.4 Histograms of the arrival-time jitter for 3 voltages when the trigger is locked to the $60 \mathrm{~Hz}$ line. 
The timing jitter is reduced by a factor of 10 to about $3 \mathrm{~ns}$. The delay between the trigger and the arrival-time is measured as a function of operating voltage. The result is shown in Figure 5. For the laser system, if the mode lock frequency is lower than $150 \mathrm{MHz}$ (corresponding to an inter-pulse spacing larger than $6 \mathrm{~ns}$ ), the trigger will have to be locked to the mode lock frequency as well.

In our specifications of our laser system (see Appendix A) we also requested the identification of sources and methods of controlling phase jitter. The laser oscillator should have an active phase-stabilizer to meet the picosecond jitter requirement. Stability in the other subsystem is also under investigation.

- The stability of the dual-frequency source (to be described next).

- Phase accumulation in CARM interaction.

- Phase accumulation in laser regen amplifier.

- The choice and arrangement of optical fiber, if used.

- Transmission of laser and RF pulses - avoid long path lengths.

\subsubsection{Design of dual-frequency source}

The design of a two-frequency synthesizer module suitable for driving our experiment is continuing. We have in house an HP CW synthesizer (HP-8671B) capable of producing stable 2 to $18 \mathrm{GHz}$ signals at $\mathrm{mw}$ levels. The synthesizer also has a 10 $\mathrm{MHz}$ reference output. Based on this equipment and the following requirements, we investigated a wide range of possible designs. The final design will be chosen based on a tradeoff between the attainable phase stability and the cost involved.

- The mode lock frequency will be a sub multiple of the microwave frequency (around $17.14 \mathrm{GHz}$ ).

- The two frequency outputs should be phase locked to within 1 ps.

- There should be a $\pm 5 \%$ range of tuning to accommodate the variations in future gun cavities.

- The available harmonic numbers and the corresponding mode locking frequencies are listed in Appendix $B$ for the case of harmonic numbers generated frow: products ot powers of 2,3 , and 5 . We prefer to use a low mode lock frequency because it provides more densely-packed usable mode lock frequencies (see Figure 6). This is especially true if every integer is made available, as shown in Figure 7.

The design procedure is more complicated than its counterpart for timing $3 \mathrm{GHs}$ photocathode RF guns. First of all, the timing tolerance is six times as stringent both in timing jitter and phase jitter. Secondly, there are less variety of available building 


\section{Linac Pulse Arrival Time}

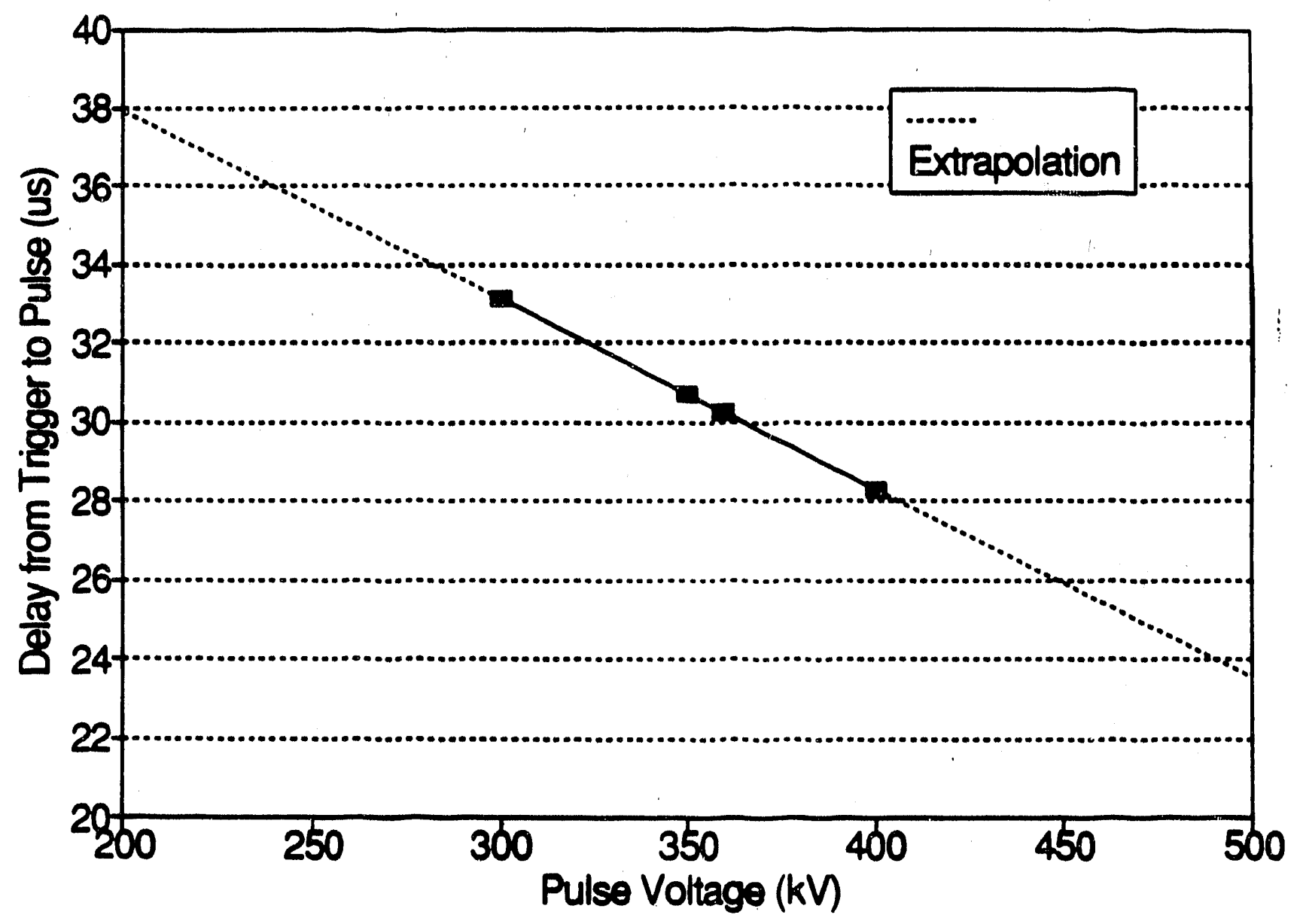

Fig.5 Arrival-time as a function of voltage. 


\section{MODELOCK FREQUENCY SPACING Simple Subharmonic \# $(2,3,5)$}

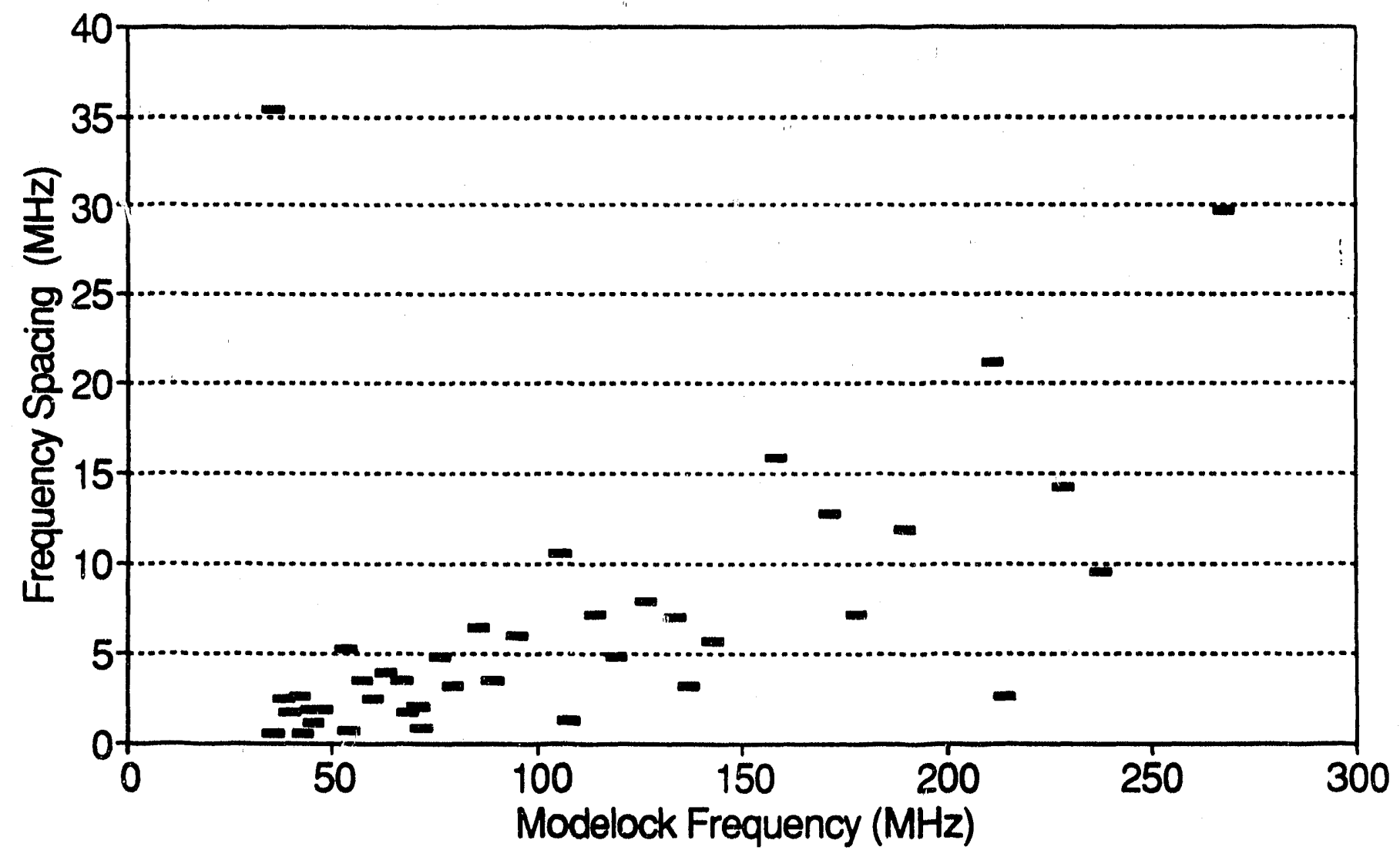

Fig. 6 The frequency spacing between available mode lock frequencies for integers generated by the product of powers of 2,3 , and 5 . 


\section{MODELOCK FREQUENCY SPACING Subharmonic \# 64-486}

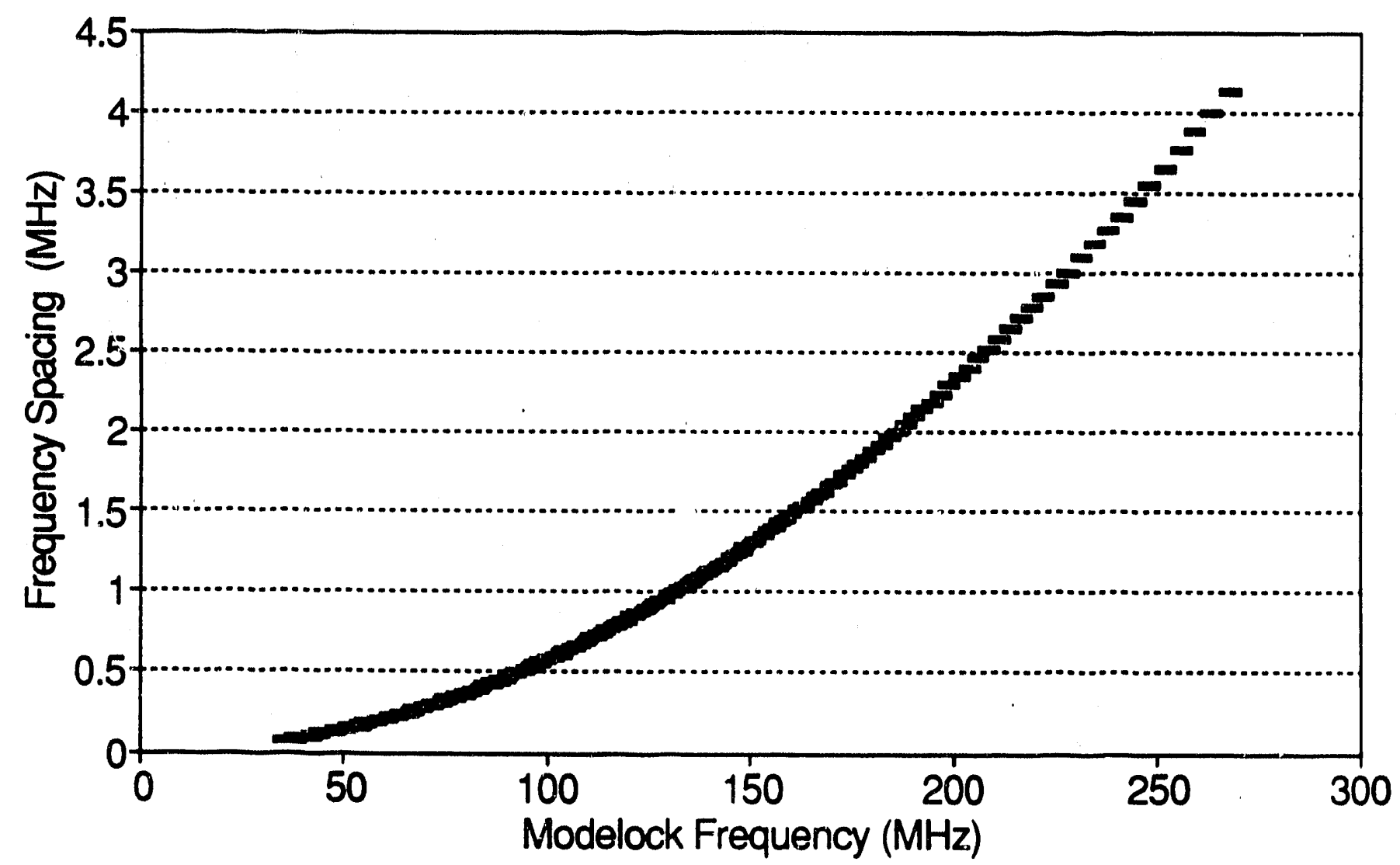

Fig.7 The frequency spacing between available mode lock frequencies for all integers between 64 and 486. 
blocks which are inexpensive (e.g. multipliers) in Ku band. Thirdly, the uncertainty in the gun cavity frequency due to finite fabrication tolerances is greater at $17 \mathrm{GHz}$.

We have investigated a variety of 2 -frequency phase-locked source approaches, and the design of the dual-frequency source will be completed once the final choice of the mode-lock frequency is provided by the laser company.

\subsubsection{Laser system}

Due to the rapid development in the field of high power short pulse laser amplifiers, we have the chance to evaluate laser systems previously were prohibitively expensive. In addition to the $\mathrm{Nd}$ :YLF system which we have been designing, the $\mathrm{Ti}: \mathrm{Sa}$ modelock laser appears to be a viable alternative. Its recent success in commercialization has brought down the price substantially. However, there is no phase-jitter control in these systems yet.

We have revised our request for quotations (RFQ) to incorporate these different types of lasiers. A copy of it can be found in Appendix A. For example, the choice of mode-lock frequency will be relaxed which allows vendors of different laser types to have rooms for maneuver in their design. This revision in RFQ introduced some delay in the laser acquisition, but we think it will payoff in the long run.

The laser system will be purchased in one of the two options. The first and preferred option is to purchase a complete system from a single vendor. The second option consists of buying the laser system as a collection of components. The second option will be chosen if the first option proves prohibitively expensive. Details of the timing and phasing considerations can be found in Appendix B.

We expect to order the laser in April 1992, and start receiving and testing the components and the system in August 1992.

\subsubsection{RF gun cavity and coupling structure}

The mechanical designs for the RF gun cavity, the waveguide coupling structure, and the vacuum pumping scheme are described as follows:

RF Gun Cavity At $17 \mathrm{GHz}$ the small physical size (and hence small heat mass) of the RF gun cavity makes temperature control easier to implement. However, the small dimension does make it difficult to implement cooling, power monitoring, and tuning systems simultaneously. We therefore incorporate the following changes:

1. For cooling system, the three water-flow loops: cathode, exit, and body loop were considered for their cooling effects. We eliminated the exit loop and replace the exit structure with a solid block for structural as well as thermal reasons (see Figure 8). In addition, to the other two loops that are kept, we plan to install an "external" cooling system to provide further cooling.

2. For monitoring and tuning system, similar to BNL design, we plan to install a monitor and a tuner for each cell of the cavity. However, due to the limitation on 
the available spaces, we introduced a scheme which offsets the angles of monitor and tuner between two cells (see Figure 9). Also, the monitor and tuner are a little larger than a simple scaling from BNL design would indicate, otherwise the tuning rods (pins) inside the tuner will be to tiny to be practical (see Figure $10)$.

We have cold-tested a prototype cavity with similar dimensions. The cavity $Q$ was found to be 951 , very close to our design valu'e at 1000 . This corresponds to a fill-time $\frac{Q}{\omega}$ of $9.5 \mathrm{~ns}$.

Waveguide coupling structure The waveguide coupling scheme cannot be directly scaled from the BNL design because the waveguide used at $17 \mathrm{GHz}$ (WR-62) is not directly scaled from that used at $3 \mathrm{GHz}$ (WR-284). However, we basically adopt the same sidewall coupling scheme to couple the $\mathrm{TE}_{10}$ waveguide mode into the cavity to excite the desired $\pi$ mode resonance (see Figure 11). The coupling site of the waveguide is cut to a circular section. The reason is two fold: one is to fit the cavity shape, the other is to provide a capacitive junction for the waveguide so that the field strength is enhanced at the junction and hence the ooupling efficiency increases. A cold test with a prototype cavity indicates that this kind of coupling schemes can reduce the reflected power down to more than $-30 \mathrm{~dB}$ !

Pumping port The major difference between our clesign and BNL's is that: our pumping port is tilted at an angle of sixty degrees from the perpendicular line to the waveguide (Figure 11). The purpose of the tilt is to make the cross section of the tube intersected by the waveguide be elliptical, other than circular, so that the effective pumping area could be twice larger. The angle also enhances the molecular flow and the pumping conductance.

A set of mechanical drawing is included in Appendix C. The cavity is currently being electroformed. We expect to receive it in February and to complete the cavitywaveguide assembly ready for integration in April, 1992.

\subsubsection{Frequency scaling}

Extensive numerical simulations have been done with the program PARMELA to show the dependence of the beam parameters, such as emittance, energy spread, peak current, and brightness on the operating frequency of the RF gun cavity.

The dimensions of the RF cavity have been scaled with the frequency, and the shape of the RF fields is kept the same. We have followed two approaches to define the strength of the on axis electric field $E_{0}$, i.e to define the parameter $\alpha$ ( $\alpha$ being the dimensionless field strength $\left.\alpha \equiv \frac{e E_{0} \lambda}{2 \pi m c^{2}}\right)$ : in the first one, $\alpha$ is kept constant, that is $E_{0}$ is proportional to the frequency $\mathrm{f}$. In the second one, we have taken one third of the breakdown RF field (which scales as $\sqrt{f}$ using SLAC results) to define $\alpha$; The latter gives the maximum available field strength for each frequency. It is believed that RF guns running with higher $\alpha^{\prime} s(\alpha \gg 1)$ produce better quality beams. This 
is because the electron bunches under high fields vill rapidly pass through the low velocity regime and suffer less beam quality degiadation. The first approach gives the same energy at the exit of the cavity for each frequency, whereas in the second one the energy is higher at low frequency. The energy $\gamma$ can be approximated by $\gamma=1+(1.5 \pi \alpha)$.

In all cases, the laser pulse length is kept constant (2ps) and the spot size is scaled with the frequency; that means that the current density (if the charge is constant) is higher at high frequency.

PARMELA solves the equation of motion for a collection of macroparticles with the RF phase as independent variable. The phase step (time step) can be chosen separately for the evaluation of the RF fields and the evaluation of the space charge effect; the Coulomb interaction between earls macroparticle is evaluated by a point to point code at each time step; this method being very time consurning (proportional to the number of particle squared) the number of macroparticle that can be simulated as well as the total number of phase steps is limited. 500 macroparticles and a phase step of one degree give a good accuracy for each frequency, with an acceptable CPU tirie for the calculation on a CRAY 2. It should be noted that PARMELA generally gives 3 to 4 times the emittance calculated by MAGIC, the other quantities being within $20 \%$. However, the general trend is the same in both programs.

We have chosen five frequencies: $476,2856,8568,17136,33000 \mathrm{MHz}$. For each frequency the RF/laser phase and the bunch charge have been varied (six different phases and six different charges). That gives 180 runs for each set of simulation or 360 runs for the two sets, or 30 hours of CPU time. As ain example, we only show some of the results on figure 12 and 13 . We have tried to extract simple heuristic laws from the simulation results to reproduce the general trend obtained for the emittance, the peak current, and the brightness, as functions of the frequency, the initial phase, and the charge. The results can be summarized as follow:

- $\alpha$ constant:

$$
\epsilon \propto \frac{Q}{f}, \quad \hat{I} \propto Q, \quad B \propto \frac{f^{2}}{Q}
$$

- $\alpha$ maximized:

$$
\epsilon \propto \frac{Q}{\sqrt{f}}, \quad I \propto Q, B \propto \frac{f}{Q}
$$

In these simulations, the transverse fields are linear in $r$, so the nonlinear RF forces due to off-axis effects are neglected. Then, the two possible sources of emittance growth are the time dependents RF effects and the space charge effects. Figure 12a shows that the space charge effects are predominant. The emittance growth always occurs in a very small distance near the cathode, where the electrons are non relativistic. If one increases $E_{0}$, the electrons are accelerated at a relativistic energy in a shorter distance, and thus the emittance growth is reduced. This is what happens when we increase the frequency: in case $1, E_{0}$ varies as $f$ and consequently $\epsilon$ varies as $\frac{1}{f}$, whereas in case $2, E_{0}$ varies as $\sqrt{f}$ and the emittance as $\frac{1}{\sqrt{f}}$. 
Fig. 8 Mechanical drawing showing the cooling loops at the cathode end and along the gun body.
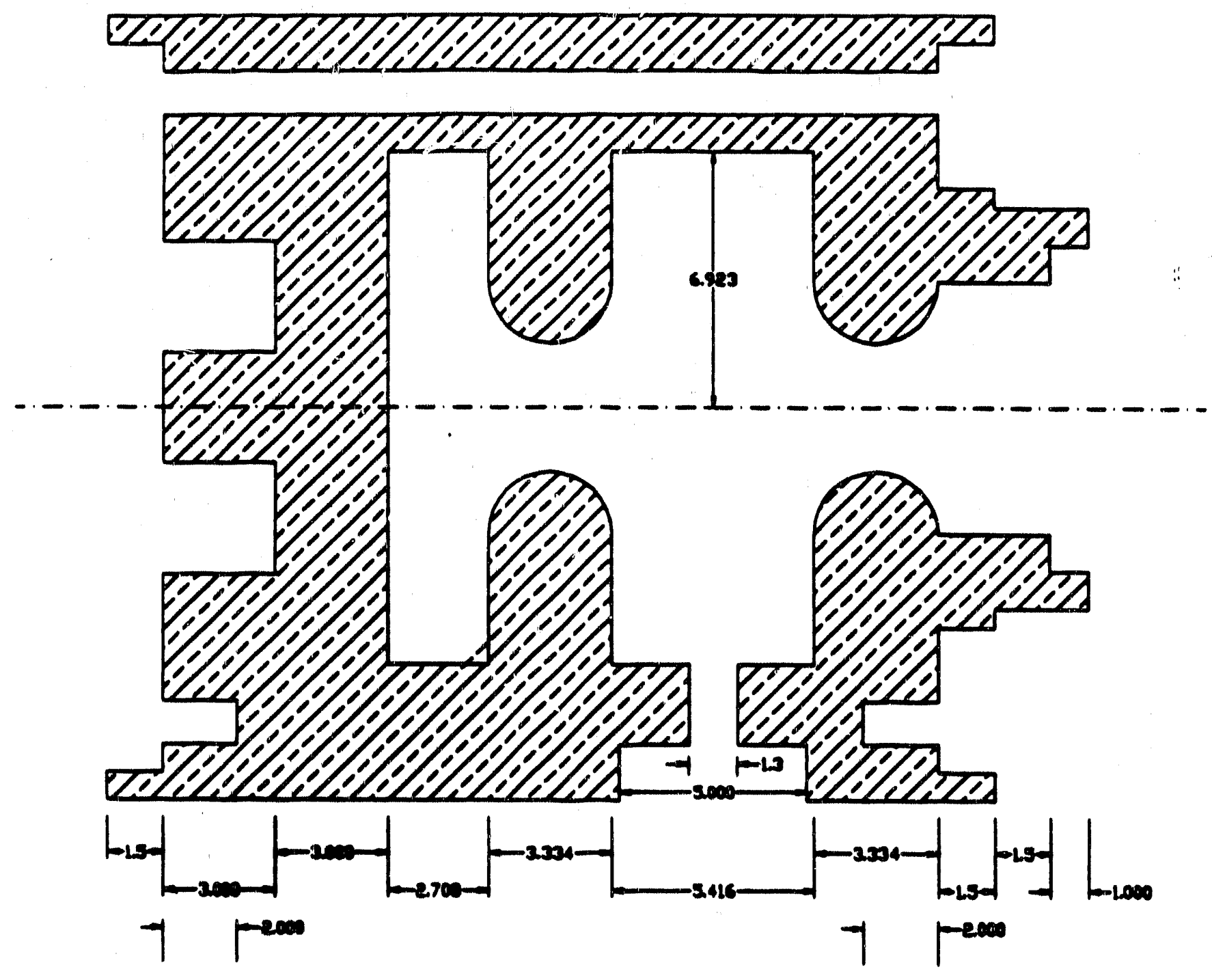

cross section DD' 
Fig.9 Mechanical drawing showing the offset scheme of the tuners and the power monitors.

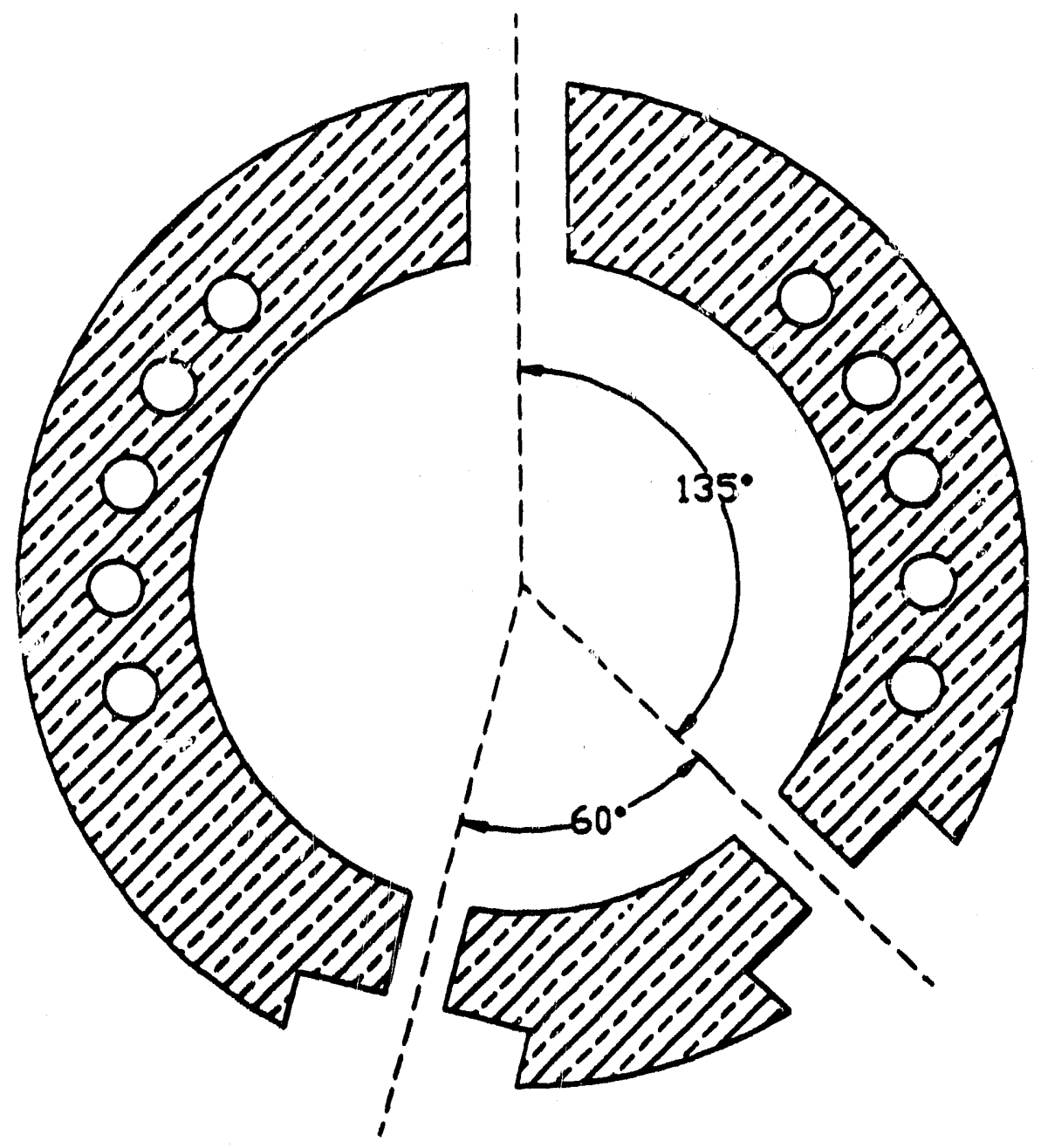

cross section $\mathrm{QU}^{\prime}$ (front view) 
Fig.10 Mechanical drawing showing the size of the tuning hole.

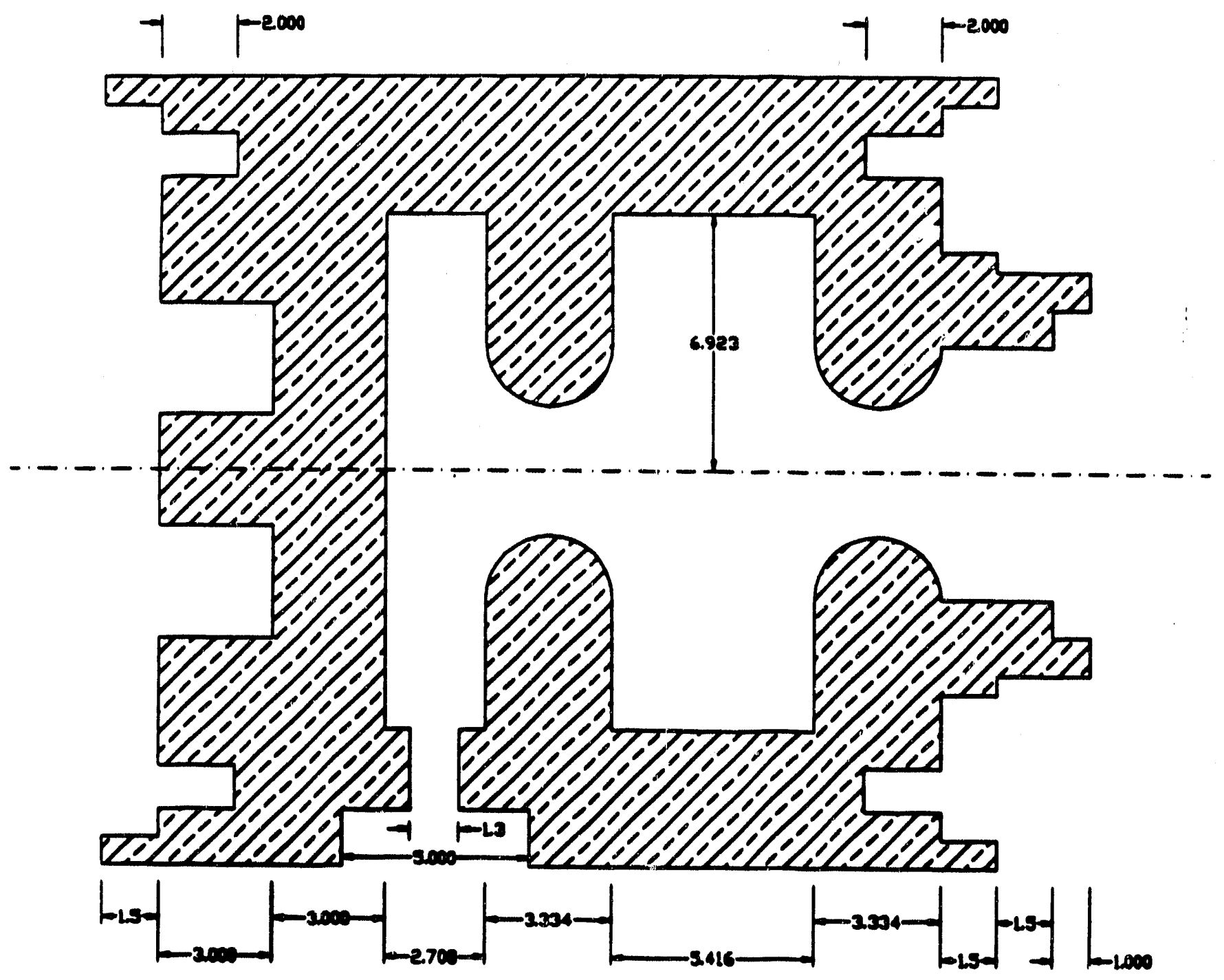

cross section $\mathrm{BB}^{\prime}$ 


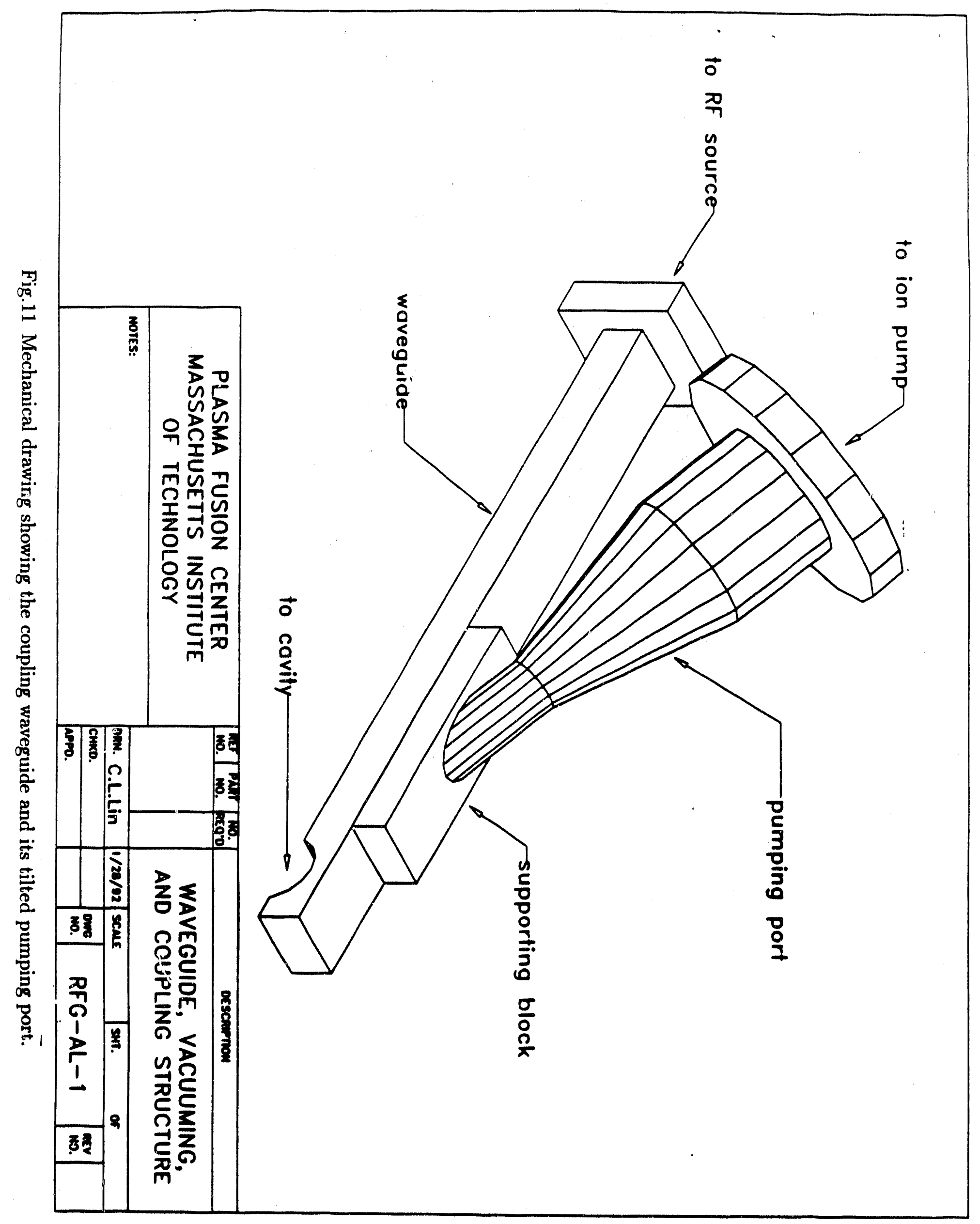


Extensive numerical simulations have been done with the program PARMELA to show the dependence of the beam parameters, such as emittance, energy spread, peak current, and brightness on the operating frequency of the RF gun cavity.

The dimensions of the RF cavity have been scaled with the frequency, and the shape of the $\mathrm{RF}$ fields is kept the same. We have followed two approaches to define the strength of the on axis electric field $E_{0}$, i.e to define the parameter $\alpha$ ( $\alpha$ being the dimensionless field strength $\left.\alpha \equiv \frac{e E_{0} \lambda}{2 \pi m c^{2}}\right)$ : in the first one, $\alpha$ is kept constant, that is $E_{0}$ is proportional to the frequency $f$. In the second one, we have taken one third of the breakdown $R F$ field (which scales as $\sqrt{f}$ using SLAC results) to define $\alpha$; The latter gives the maximum available field strength for each frequency. It is believed that RF guns running with higher $\alpha$ 's $(\alpha \gg 1)$ produce better quality beams. This is because the electron bunches under high fields will rapidly pass through the low velocity regime and suffer less beam quality degradation. The first approach gives the same energy at the exit of the cavity for each frequency, whereas in the second one the energy is higher at low frequency. The energy $\gamma$ can be approximated by $\gamma=1+(1.5 \pi \alpha)$.

In all cases, the laser pulse length is kept constant (2ps) and the spot size is scaled with the frequency; that means that the current density (if the charge is constant) is higher at high frequency.

PARMELA solves the equation of motion for a collection of macroparticles with the RF phase as independent variable. The phase step (time step) can be chosen separately for the evaluation of the RF fields and the evaluation of the space charge effect; the Coulomb interaction between each macroparticle is evaluated by a point to point code at each time step; this method being very time consuming (proportional to the number of particle squared) the number of macroparticle that can be simulated as well as the total number of phase steps is limited. 500 macroparticles and a phase step of one degree give a good accuracy for each frequency, with an acceptable CPU time for the calculation on a CRAY 2. It should be noted that PARMELA generally gives 3 to 4 times the emittance calculated by MAGIC, the other quantities being within $20 \%$. However, the general trend is the same in both programs.

We have chosen five frequencies: $476,2856,8568,17136,33000 \mathrm{MHz}$. For each frequency the RF/laser phase and the bunch charge have been varied (six different phases and six different charges). That gives 180 runs for each set of simulation or 360 runs for the two sets, or 30 hours of CPU time. As an example, we only show some of the results on figure 12 and 13. We have tried to extract simple heuristic laws from the simulation results to reproduce the general trend obtained for the emittance, the peak current, and 


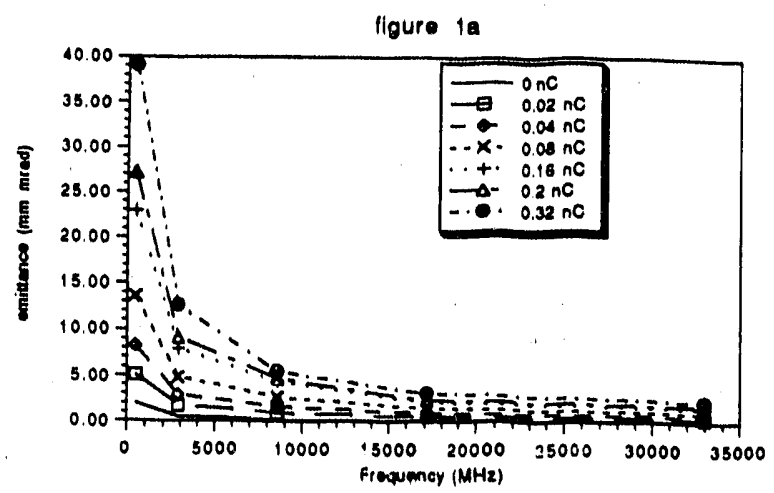

Fig.12 (a) emittance versus frequency for $\phi_{0}=20$ degrees, $\alpha=$ constant. Each curve is for different charges.

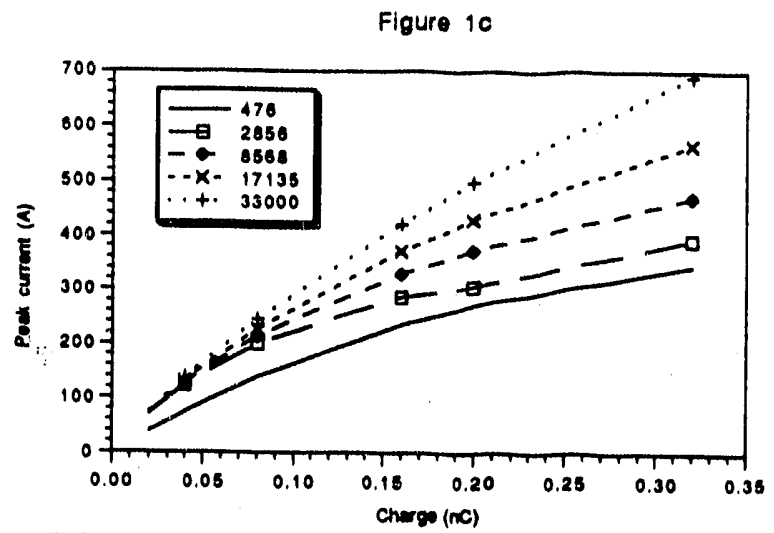

(c) peak current versus charge for $\phi_{0}=20$ degrees, $\alpha=$ constant. Each curve is for different frequency.

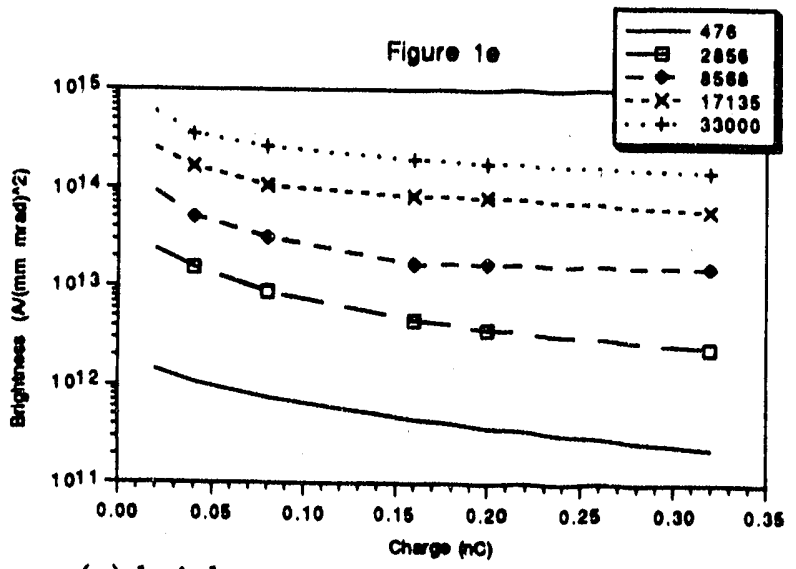

(e) brightness versus charge for $\phi_{0}=20$ degrees, $\alpha=$ constant. Each curve is for different frequency.
- Figure 16

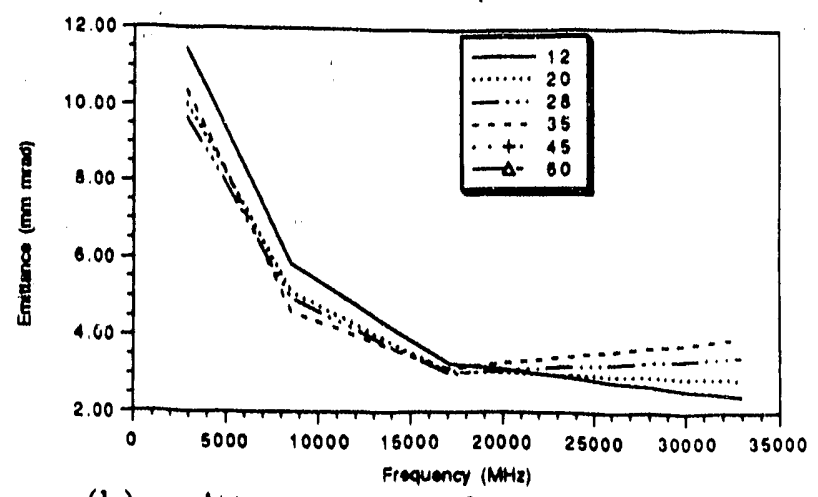

(b) emittance versus frequency for $Q=0.32$ $\mathrm{nC}, \alpha$ maximum for each frequency. Each curve is for different $\phi_{0}$.

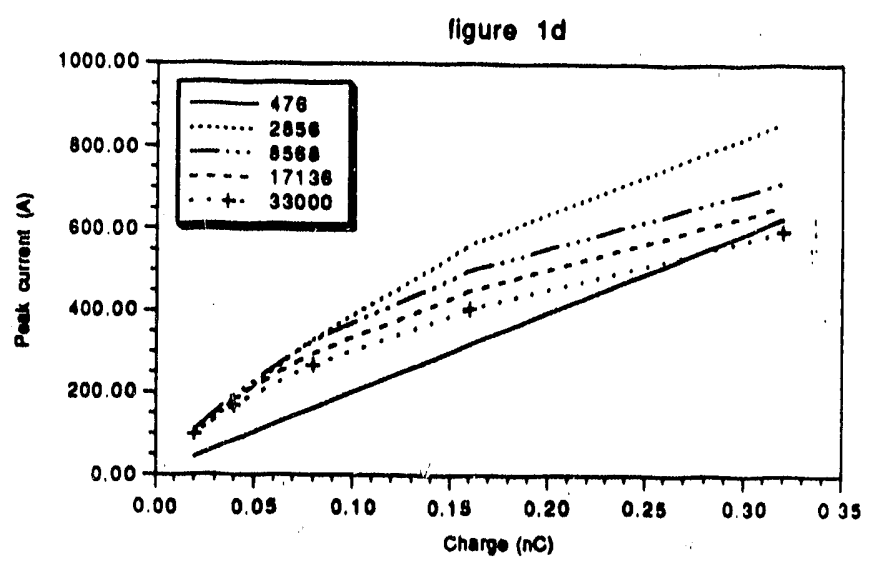

(d) peak current versus charge for $\phi_{0}=20$ degrees, $\alpha$ maximum for each frequency. Each curve is for different frequency.

Figure 11

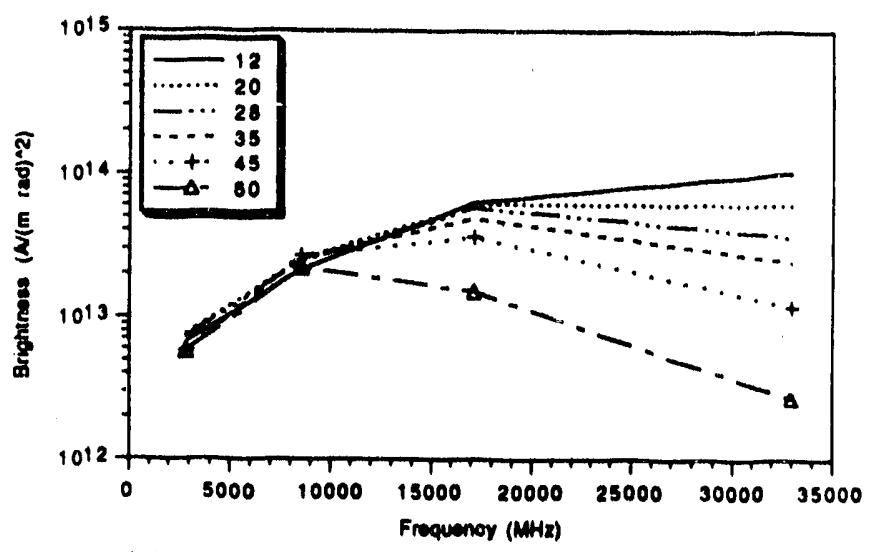

(f) brightness versus frequency for $Q=0.32$ nC, $\alpha$ maximum for each frequency. Each curve is for different $\phi_{0}$. 


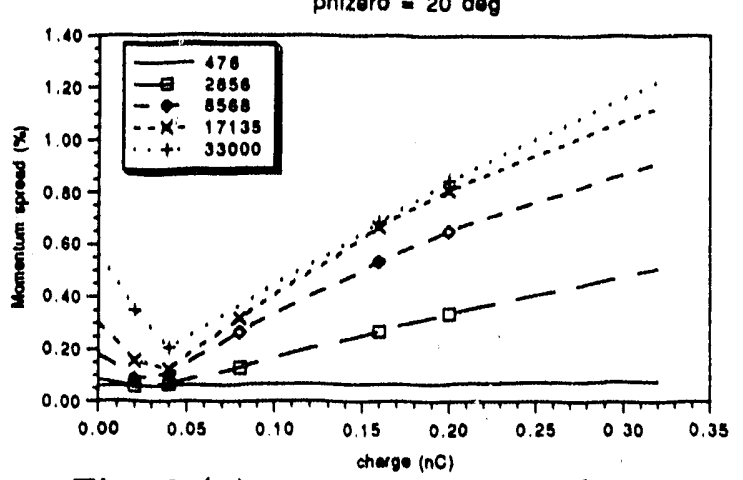

Fig.13 (a) momentum spread versus charge for $\phi_{0}=20$ degrees,
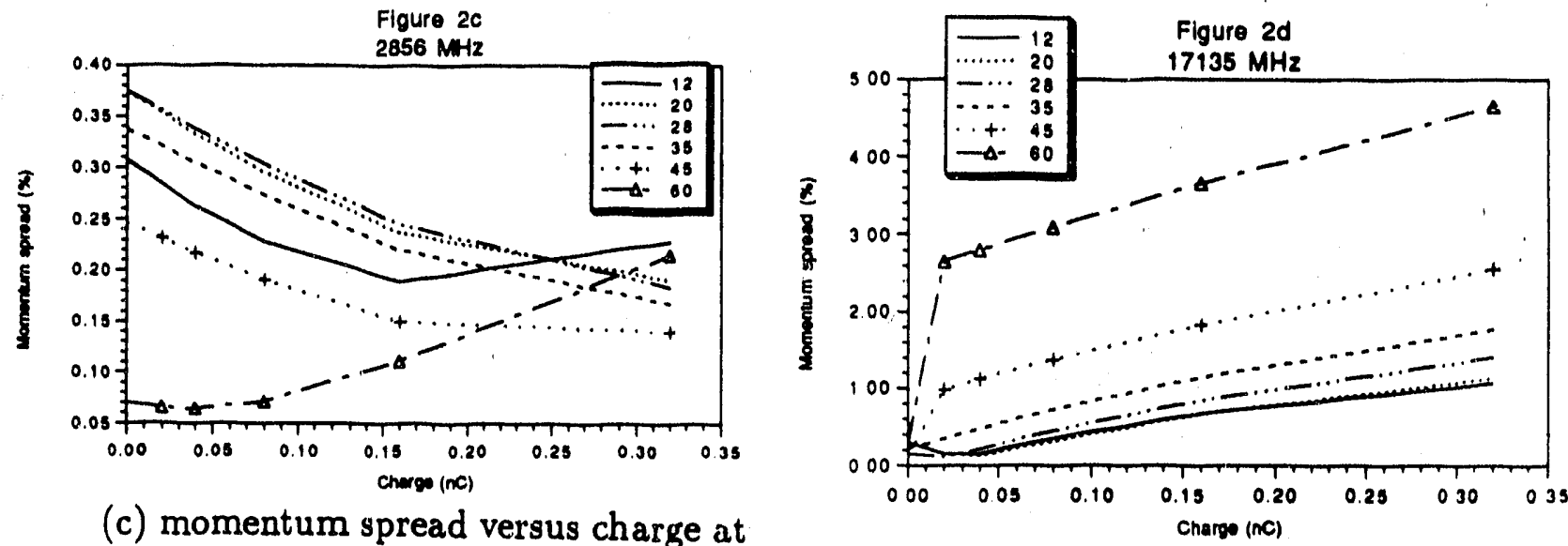

(c) momentum spread versus charge at $3 \mathrm{GHz}$ for six initial phases,

(d) momentum spread versus charge at $17 \mathrm{GHz}$ for six initial phases. for $\phi_{0}=35$ degrees,

Figure $2 b$

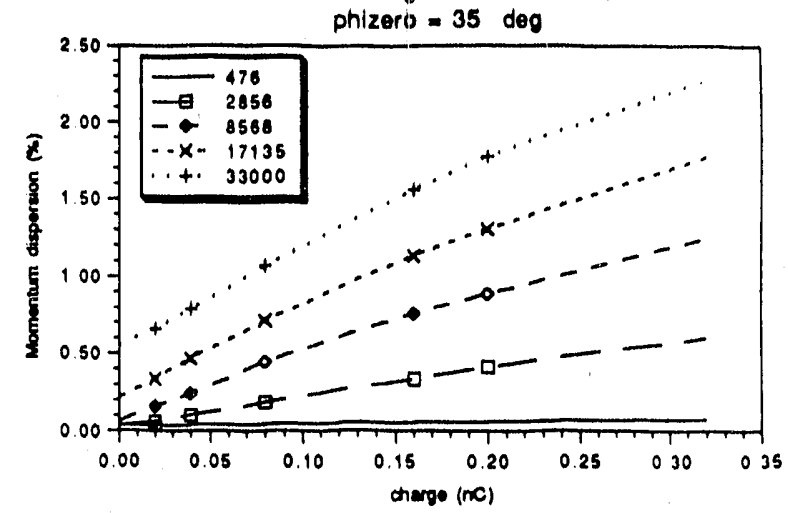

(b) momentum spread versus charge 
the brightness, as functions of the frequency, the initial phase, and the charge. The results can be summarized as follow:

$-1 \alpha=$ constant

$$
\epsilon \propto \frac{Q}{f}, \quad \hat{I} \propto Q, \quad B \propto \frac{f^{2}}{Q}
$$

$-2 \alpha$ maximum

$$
\epsilon \propto \frac{Q}{\sqrt{f}}, \hat{I} \propto Q, B \propto \frac{f}{Q}
$$

In these simulations, the transverse fields are linear in $r$, so the nonlinear RF forces due to off-axis effects are neglected. Then, the two possible sources of emittance growth are the time dependents RF effects and the space charge effects. Figure 12a shows that the space charge effects are predominant. The emittance growth always occurs in a very small distance near the cathode, where the electrons are non relativistic. If one increases $E_{0}$, the electrons are accelerated at a relativistic energy in a shorter distance, and thus the emittance growth is reduced. This is what happens when we increase the frequency: in case $1, E_{0}$ varies as $f$ and consequently $\epsilon$ varies as $\frac{1}{f}$, whereas in case $2, E_{0}$ varies as $\sqrt{f}$ and the emittance as $\frac{1}{\sqrt{f}}$.

In case 1 , the bunch length varies as $\frac{1}{f}$, and thus the peak current varies as $f$. This is not due to time dependent $R F$ effects since there is no variation when $Q=0$. Retaining the arguments for the emittance, one can say that the bunch lengthening due to the space charge effects is less important at high frequency (that is at higher field).

If the brightness is sole figure of merit of the RF gun, one can conclude that it is interesting to work at low charge and high frequency.

The behaviour for the energy spread is more complicated and it is difficult to extract a simple law. Figure 13 shows the variation of the momentum spread with the charge; one can clearly see two domains: at low charge the momentum spread decreases, reaches a minimum at $0.04 \mathrm{nC}$, and then increases almost linearly with the charge. If $\phi_{0}$ is increased, this effect disappears and the momentum spread becomes a linear function of the charge.

The nonlinear RF forces, which are neglected in these simulations, are important when the beam radius becomes large with respect to the cavity aperture. This happens mainly at high frequency when the particles are near the exit aperture. The effects of the nonlinear RF forces will be included in MAGIC simulations to be conducted in the near future. The MAGIC results will be compared with the present PARMELA results.

\subsection{Beam transport and diagnostic line}


The purposes of the beam transport (and diagnostic) line is to bend away the electrons exiting the RF cavity from the laser window, and to measure the electron beam parametcrs. The quantities to measure are energy, energy spread, transverse emittance, charge, and bunch length.

Figure 14 shows the schematic diagrain of the beam line including a quadruple triplet and a bending magnet.

The bending magnet bends the electrons by an angle of 90 degrees and form a point to point imaging system that can be used for energy spi ead measurement. A very small beam size is produced before the bending magnet (object plane), with the proper quadrupole setting, and the bending magnet produces a spot on a fluorescent screen in the image plane. The fluorescent spot is observed with a video camera. The position of the spot gives the energy and its horizontal thickness the energy spread. It can be shown that the energy resolution, tha'c is the smallest measurable energy spread, is, in this configuration, given by

$$
\delta_{R}=\frac{D}{\rho(\rho+D)} \sigma_{r 0}
$$

where $D$ is the distance from the exit plane of the bending magnet to the image plane, $\rho$ is the radius of curvature of the bending magnet, and $\sigma_{x 0}$ is the natural beam size (horizontal) of the electron beam in the object plane. To reach a small resolution, one must then choose $\rho$ large and D small, and produce a minimum beam size in the object plane. The former is linuited by space considerations, and the later by the finite emittance of the electron beam. A quadrupole triplet placed between the RF cavity and the bending magnet produces the waist (object plane) whose position and size can be varied.

The quadrupole triplet can also be used to measure the emittance: the bending magnet is switched off and we observe the spot produced by the electrons on another screen positioned along the RF gun axis. The gradient (K) of one of the quadrupole is varied in order to vary the spot size on the screen; a least square analysis of the curve $\sigma_{x, y}$ vs. K gives the transverse emittance.

The program TRACE3d, which uses the transport formalism was used to obtain this preliminary design; the beam parameters in this simulation are:

$$
\epsilon=1.8 \pi \mathrm{mm} \operatorname{mrad}, \mathrm{r}=0.56 \mathrm{~mm}, \theta_{r}=22 \mathrm{mrad}, \frac{\Delta p}{p}=0.44 \%, \mathrm{Q}=0.1 \mathrm{nC} \text {. }
$$

Figure 15 shows the transverse and longitudinal phase space and the beam envelope. Figure $15 \mathrm{a}$ is the transverse phase space at the exit of the avity, and is used as initial conditions for TRACE3d. Figure 15b represents the transverse phase space at the end of the line, that is at the fluorescent screen. The solid and dashed lines stand for $x, \theta_{x}$, and $y, \theta_{y}$ planes respectively. The two eilipses are upright (no divergence) and one can see that 


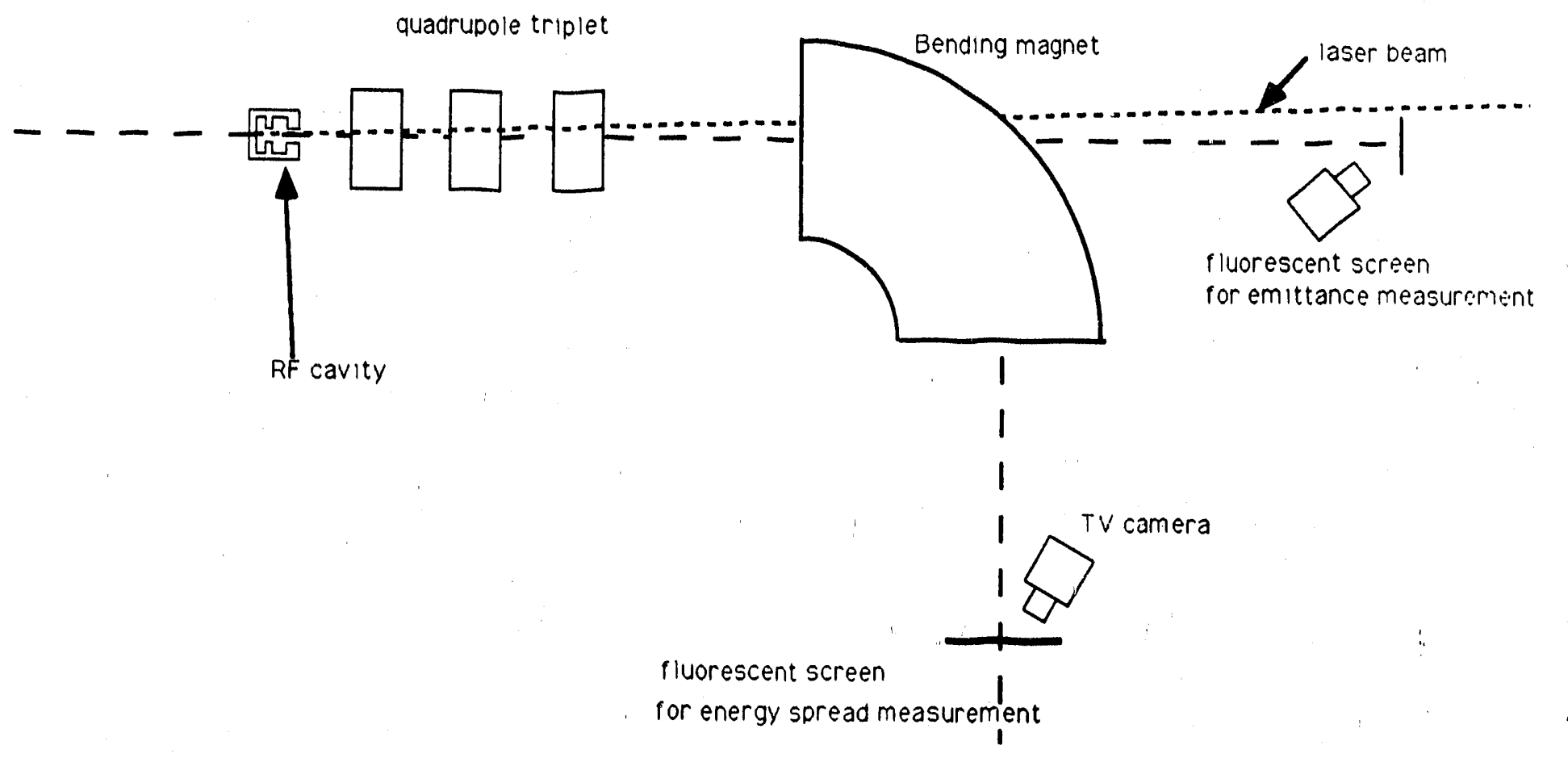

Fig.14 Schematic of the beam transport and diagnostic line 

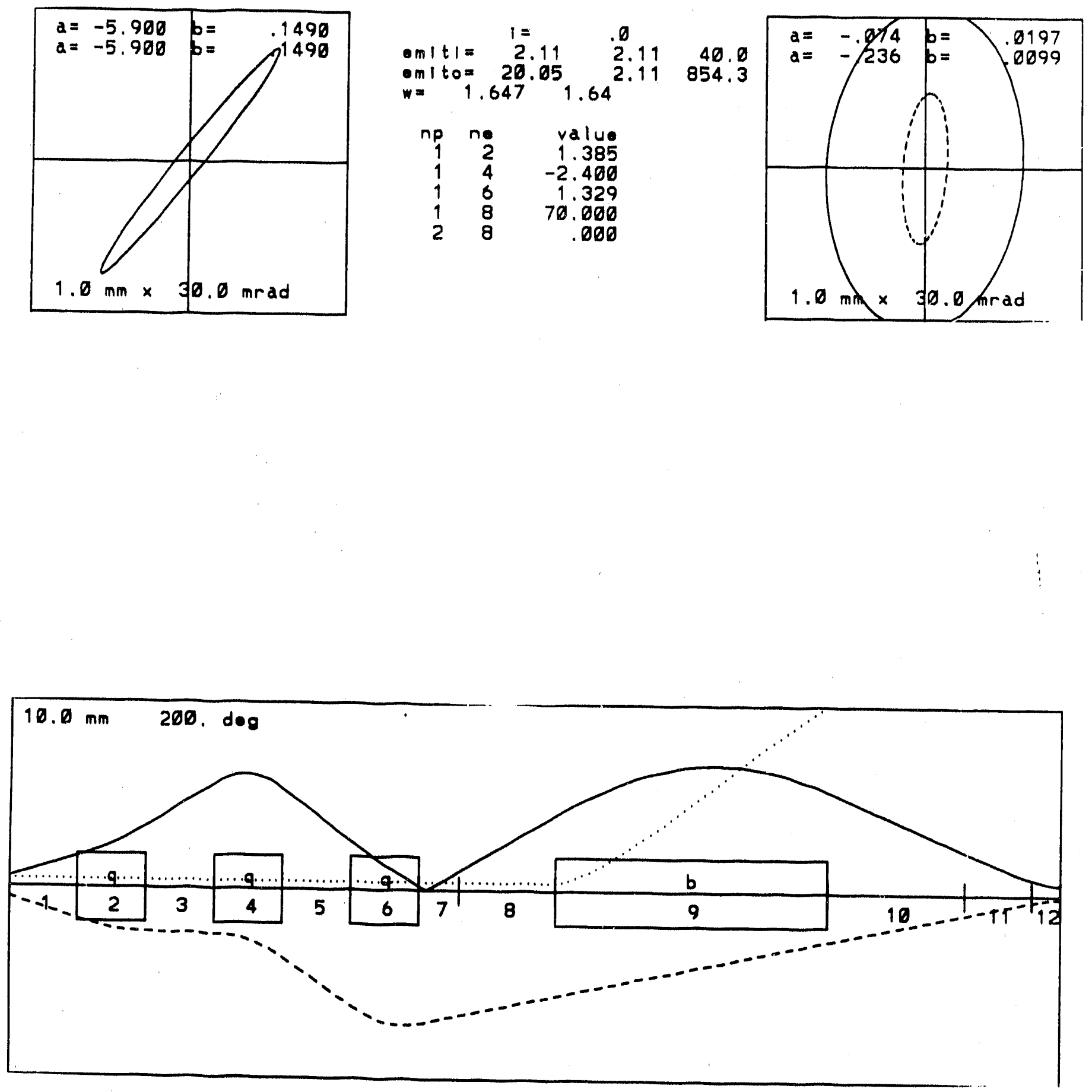

Fig.15 Transverse and longitudinal phase space and the beam envelope 
In case 1 , the bunch length varies as $\frac{1}{f}$, and thus the peak current varies as $f$. This is not due to time dependent $R F$ effects since there is no variation when $Q=0$. Retaining the arguments for the emittance, one can say that the bunch lengthening due to the space charge effects is less important at high frequency (that is at higher field).

If the brightness is sole figure of merit of the RF gun, one can conclude that it is interesting to work at low charge and high frequency.

The behaviour for the energy spread is more complicated and it is difficult to extract a simple law. Figure 13 shows the variation of the momentum spread with the charge; one can clearly see two domains: at low charge the momentum spread decreases, reaches a minimum at $0.04 \mathrm{nC}$, and then increases almost linearly with the charge. If $\phi_{0}$ is increased, this effect disappears and the momentum spread becomes a linear function of the charge.

The nonlinear RF forces, which are neglected in these simulations, are important when the beam radius becomes large with respect to the cavity aperture. This happens mainly at high frequency when the particles are near the exit aperture. The effects of the nonlinear RF forces will be included in MAGIC simulations to be conducted in the near future. The MAGIC results will be compared with the present PARMELA results.

\subsubsection{Beam transport and diagnostic line}

The purposes of the bean transport (and diagnostic) line is to bend away the electrons exiting the RF cavity from the laser window, and to measure the electron beam parameters. The quantities to measure are energy, energy spread, trans irse emittance, charge, and bunch length.

Figure 14 shows the schematic diagram of the beam line including a quadruple triplet and a bending magnet.

The bending magnet bends the electrons by an angle of 90 degrees and form a point to point imaging system that can be used for energy spread measurement. A very small beam size is produced before the bending magnet (object plane), with the proper quadrupole setting, and the bending magnet produces a spot on a fluorescent screen in the image plane. The fluorescent spot is observed with a video camera. The position of the spot gives the energy and its horizontal thickness the energy spread. It can be shown that the energy resolution, that is the smallezt measurable energy spread, is, in this configuration, given by

$$
\delta_{R}=\frac{D}{\rho(\rho+D)} \sigma_{r 0}
$$

where $D$ is the distance from the exit plane of the bending magnet to the image plane, $\rho$ is the radius of curvature of the bending magnet, and $\sigma_{x 0}$ is the natural beam size (horizontal) of the electron beam in the object plane. To reach a small resolution, one must then choose $\rho$ large and D small, and produce a minimum beam size in the object plane. The former is limited by space considerations, and the later by the 
finite emittance of the electron beam. A quadrupole triplet placed between the RF cavity and the bending magnet produces the waist (object plane) whose position and size can be varied.

The quadrupole triplet can also be used to measure the emittance: the bending magnet is switched off and we observe the spot produced by the electrons on another screen positioned along the RF gun axis. The gradient (K) of one of the quadrupole is varied in order to vary the spot size on the screen; a least square analysis of the curve $\sigma_{x, y}$ vs. $\mathrm{K}$ gives the transverse emittance.

The program TRACE3d, which uses the transport formalism was used to obtain this preliminary design; the beam parameters in this simulation are:

$\epsilon=1.8 \pi \mathrm{mm} \mathrm{mrad}, \mathrm{r}=0.56 \mathrm{~mm}, \theta_{r}=22 \mathrm{mrad}, \frac{\Delta p}{p}=0.44 \%, \mathrm{Q}=0.1 \mathrm{nC}$.

Figure 15 shows the transverse and longitudinal phase space and the beam envelope. Figure $15 \mathrm{a}$ is the transverse phase space at the exit of the cavity, and is used as initial conditions for TRACE3d. Figure 15b represents the transverse phase space at the end of the line, that is at the fluorescent screen. The solid and dashed lines stand for $\mathrm{x}, \theta_{x}$, and $\mathrm{y}, \theta_{y}$ planes respectively. The two ellipses are upright (no divergence) and one can see that the $x$ dimension is much larger than the $y$ one due to the dispersion. The envelope of the beam is plotted on figure 15c. The solid and dashed lines stand for $\mathrm{x}$ and $\mathrm{y}$ respectively. The maximum scale is $1 \mathrm{~cm}$ in the vertical direction and $78 \mathrm{~cm}$ in the horizontal one. The quadrupoles are $5 \mathrm{~cm}$ long and spaced by $5 \mathrm{~cm}$. The maximum gradient is $2.3 \mathrm{~T} / \mathrm{m}$ for en energy of $2.3 \mathrm{MeV}$. The radius of curvature of the bending magnet is $12.7 \mathrm{~cm}$, leading to a total length of $20 \mathrm{~cm}$. The magnetic field is then 600 Gauss. The maximum transverse beam size is $7 \mathrm{~mm}$ which is well bellow the radius of the vacuum chamber which is $2 \mathrm{~cm}$. The minimum beam size obtained in the object plane is $\sigma_{x 0}=0.046 \mathrm{~mm}$ which leads to a resolution of $\delta_{R}=$ $0.02 \%$ for $D=18 \mathrm{~cm}$. The total length from the RF cavity to the screen is $1 \mathrm{~m}$.

Simulations of the same line with the program PARMELA show good agreement.

The charge will be measured with a Faraday cup placed between the quadrupole triplet and the dipole. Several methods for measuring the bunch length are under investigation.

\subsubsection{Vacuum design}

The vacuum in the RF gun, coupling waveguide, and the beamline is supported by two pumping stations. The first pumping line is located downstream at the electron beamline. The second pumping line is located at the back of the coupling waveguide. The geometries and the calculations of pumping conductance for both pump lines are included in Appendix C. With 8 liter/second pumps at both stations, the effective pumping rate is about 1 liter/second from each pump. The long section of transport waveguide and mode converters will be pumped by a third pump near the waveguide window. The pumping capacity will be calculated once the design of the mode converter and transport waveguide geometries are determined. 


\subsubsection{R.F transport and mode conversion}

We plan to rely on transit time to isolate the RF gun from CARM amplifier. The RF transport line between the CARM output window and the RF gun cavity therefore will have to be no shorter than $15 \mathrm{ft}$. The dimension of this line will be either 1 or 2 inch inner diameter. The final choice depends on the breakdown strength and the cost associated.

We are in the process of designing the mode converters between the CARM circular $T E_{11}$ mode and the linearly-polarized rectangular $T E_{10}$ mode in the coupling waveguide. The conversion process will involve a "linearizer" with a circular-ellipticalcircylar crosssection and a mode converter with a circular-rectangular crosssection. Both pieces will be elctroformed using OFHC copper.

The status of the CARM amplifier is detailed in Appendix D.

\subsection{Summary}

- The timing scheme between the CARM and the laser system has been finalized, sources of timing-jitter and phase-jitter are identified. Methods for jitter control are either being implemented or under investigation.

- The phase-locked dual-frequency source parameters are determined.

- The request for quotation for the UV ps laser system has been revised to include other promising types of lasers. The laser system will be ordered in April, 1992. Laser testing is expected to start in August, 1992.

- The RF gun cavity incorporated changes necessary for liigh frequency operations. It is currently under fabrication. We expect to receive the cavity in February. The coupling structure is also under fabrication.

- The performance of RF guns at different frequencies (500 $\mathrm{MHz}$ to $33 \mathrm{GHz}$ ) are studied using PARMELA. Preliminary results at low bunch charge show that beam quality is better at high frequencies. Preliminary frequency scaling of beam parameters are obtained.

- Preliminary transport and diagnostic line are designed using TRACE3d and checked with PARMELA. The results agree well with each other.

- The design of vacuum system, RF transport, and mode converters are underway.

The first integration period is scheduled $: 0$ be May - July, 1992. The hot test of the $17 \mathrm{GHz}$ RF gun cavity (without the laser) will follow. 


\section{Studies of High Gradient Acceleration at $33 \mathrm{GHz}$}

The experimental program on testing the LBL/Haimson high gradient structure is well under way. The power source for these studies is a $33 \mathrm{GHz}$ free-electron laser operating at $27 \%$ efficiency. This is the highest untapered FEL efficiency yet achieved. The structure tests have commenced and, at present, breakdown in the converter region between the FEL and the structure, previously a problem, has been solved. A spectrometer has been designed and fabricated to measure the energy of electrons accelerated from dark current.. The FEL is described briefly in Sec. 3.1 and in detail in Appendix E. The structure testing program is described in Sec. 3.2.

\subsection{A High Power Free-Electron Laser for Accelerator Sys- tems}

The free electron laser (FEL) operating in a combined axial guide magnetic field and a helical wiggler field has been studied experimentally and theoretically over a period of many years, in both the linear and the nonlinear regimes. In all these studies, the axial magnetic field $\mathbf{B}_{\boldsymbol{z}}$ is oriented so that it enhances the rotation of the beam electrons in the helical wiggler field $\mathbf{B}_{w}$. The increase of the transverse electron velocity $v_{\perp}$, cornpared to what it would be in the absence of $\mathbf{B}_{z}$, has, from the results of numerical simulation, potential benefits such as an enhanced radiation growth rate and efficiency. Indeed, when the cyclotron wavelength in the axial field $\lambda_{c}=2 \pi v_{z} / \Omega_{z}$ approaches the wiggler periodicity $\lambda_{w}$, the transverse electron excursions can become too large, the electrons strike the drift tube wall and are lost. $\left(\Omega_{z}=e \mathrm{~B}_{z} / \mathrm{m}_{0} \gamma\right.$ is the cyclotron frequency in the guide field and $\gamma=\left[1-\left(v_{z} / c\right)^{2}-\left(v_{\perp} / c\right)^{2}\right]^{-1 / 2}$ is the relativistic energy factor.) Thus, the "resonance" $\lambda_{c}=\lambda_{w}$ becomes a dividing line for conventional FEL operation: at relatively weak axial fields, $\lambda_{c}>\lambda_{w}$, we have the so called Group I regime, and for stronger fields such that $\lambda_{c}<\lambda_{w}$, the Group II regime. Cornparisons of experiment and theory have shown that the guide field must be included in calculating the perpendicular velocity when the beam is near resonance. Other consequences of operating near resonance, which may tend to diminish the predicted efficiency enhancements, are an increased beam radius (from the higher $v_{\perp}$ ), and thus greater detuning spread from the variation of the wiggler across the beam, and a greater sensitivity to alignment errors in the system.

We have recently reported [2] on a new regime of free electron laser operation using a helical wiggler field and a reversed axial guide magnetic field. The orientation of the axial field is such as to oppose the rotation of the electrons imparted by the helical field. A $33.3 \mathrm{GHz}$ free electron laser amplifier is driven by a mildly relativistic electron beam $(750 \mathrm{kV}, 300 \mathrm{~A}, 30 \mathrm{~ns})$ and generates $61 \mathrm{MW}$ of radiation with a $27 \%$ conversion efficiency. The FEL performance is enhanced when compared with results obtained when the axial guide field is in its conventional orientation.

The rotation of the electrons in the helical wiggler field $\mathbf{B}_{w}$ is now opposed by the presence of the guide field $\mathbf{B}_{z}$ and there is no longer the resonance at $\lambda_{c}=\lambda_{w}$. 
The transverse electron velocity $v_{\perp}$ is diminished compared to what it would be in the absence of $\mathbf{B}_{z}$, however the latter reduction is partially compensated for in our experiments by increasing $\mathbf{B}_{w}$. The reversal of $\mathbf{B}_{\boldsymbol{z}}$ yields higher radiation intensity and efficiency compared to what we were able to achieve with the conventional orientation of the axial magnetic field.

During the during the of this grant additional measurements of the spectral bandwidth and frequency of the FEL output have been made. It has been found that the in the reversed field configuration the FEL power is centered around the frequency of the injection magnetron, as expected0. For the group I operation, however, the central frequency is slightly shifted from the maximum. This phenomenum is, as yet, unexeplained theoretically. There are, however, some indications that a possible explanation may be found through a careful time-dependent analysis of the FEL interaction, including finite pulse effects and the voltage and current profiles. A discussion of these measurements can be found Appendix E.

\section{3,2 Haimson/LBL High Gradient Structure Test Program}

Substantial preparations have been rnade for the testing of the structure. These are described briefly below:

\subsubsection{RF transport to the high gradient structure}

The connections between the FEL and the high gradient accelerator (HGA) structure have been completed. The experimental setup is shown in Fig. 16. The connections include the required vacuum system and microwave components. A short piece of "squeezed" circular guide has been installed at the output end of the FEL drift tube to convert the electromagnetic wave from circular to linear polariztion. A second converter section changes from circular to rectangular waveguide. High power has been successfully transported through these converters to the entrance to the high gradient structure. A typical pulse is shown in Fig. 17, where the transimitted FEL power is plotted as a function of time in the upper curve and the reflected power (as measured back near the magnetron) is plotted in the lower trace. The reflected power is of order one percent of the transmitted power, which, for the trace shown in Fig. 17 , is approximately $60 \mathrm{MW}$.

The HGA input and output waveguides are sealed by ceramic windows and the structure is kept under vacuum by means of three ion pumps. THe waveguide connecting the FEL and the HGA is evacuted by the same vacuum pumps as are used on the FEL drift tube. After traversing the HGA, the rf power is dumped into a microwave anechoic chamber by means of a large rectangular horn. The microwave connecting the HGA to this emitting horn is also kept in vacuum by a diffusion pump. This is needed to prevent arcing at the ceramic window.

Photomultiplier tubes have been installed to monitor the possible onset of electric breakdown inside the HGA and at the input coupler of the structure. A magnetic spectrometer has been designed and fabricated to analyze the energy spectrum of the 


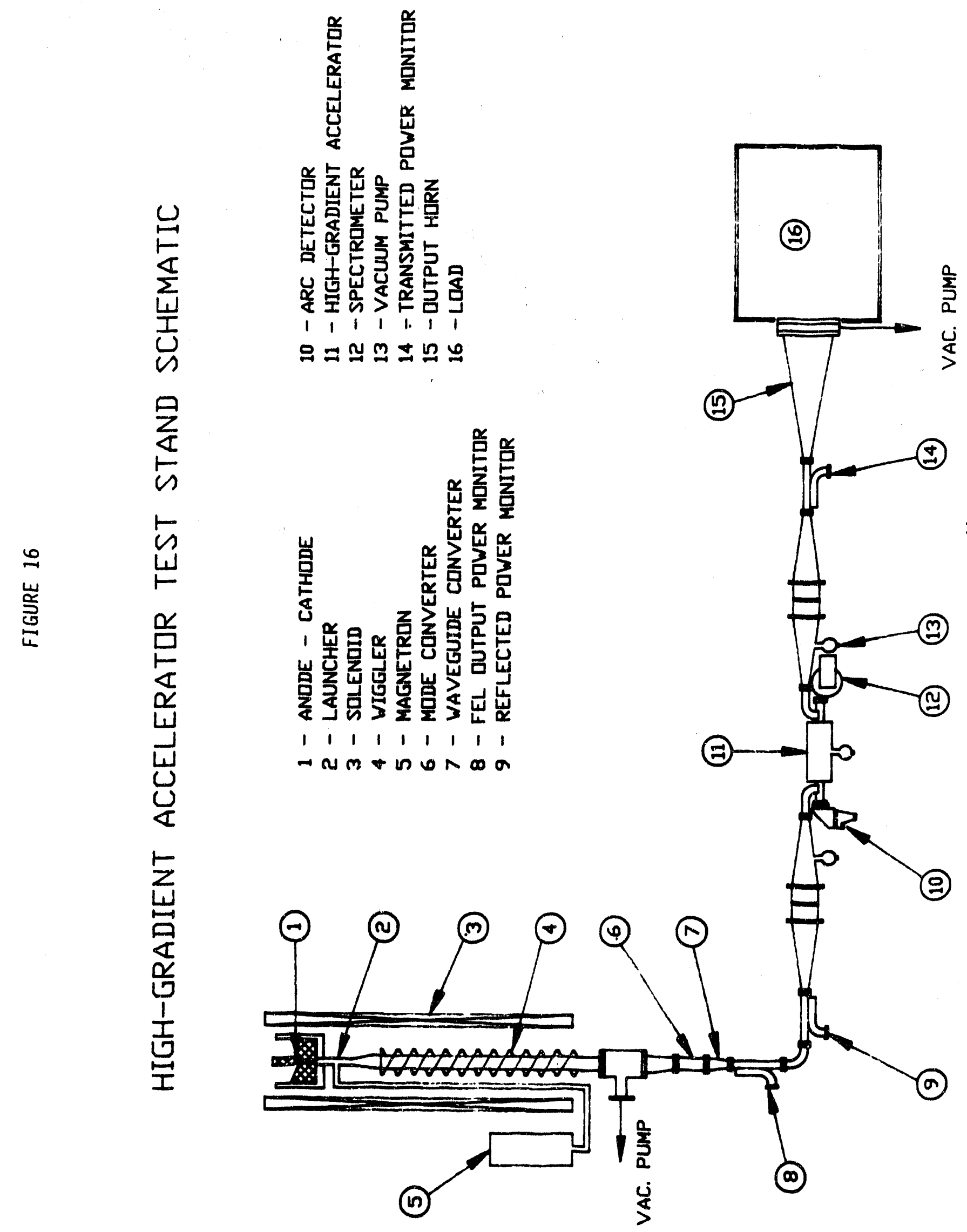




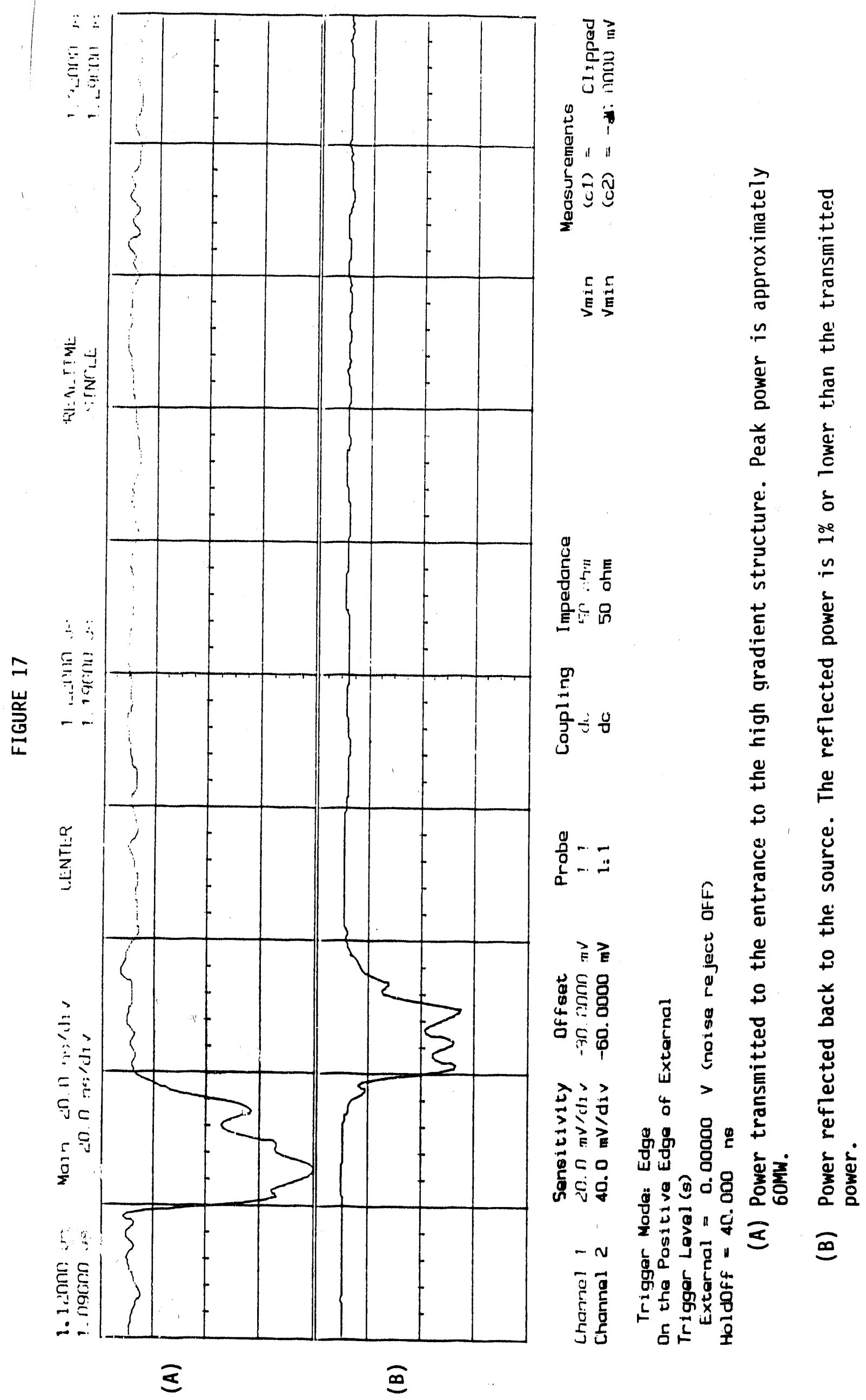




\section{FIGURE 18}

Block Dlagram of 20 MoV Eleotron Energy Analyzer

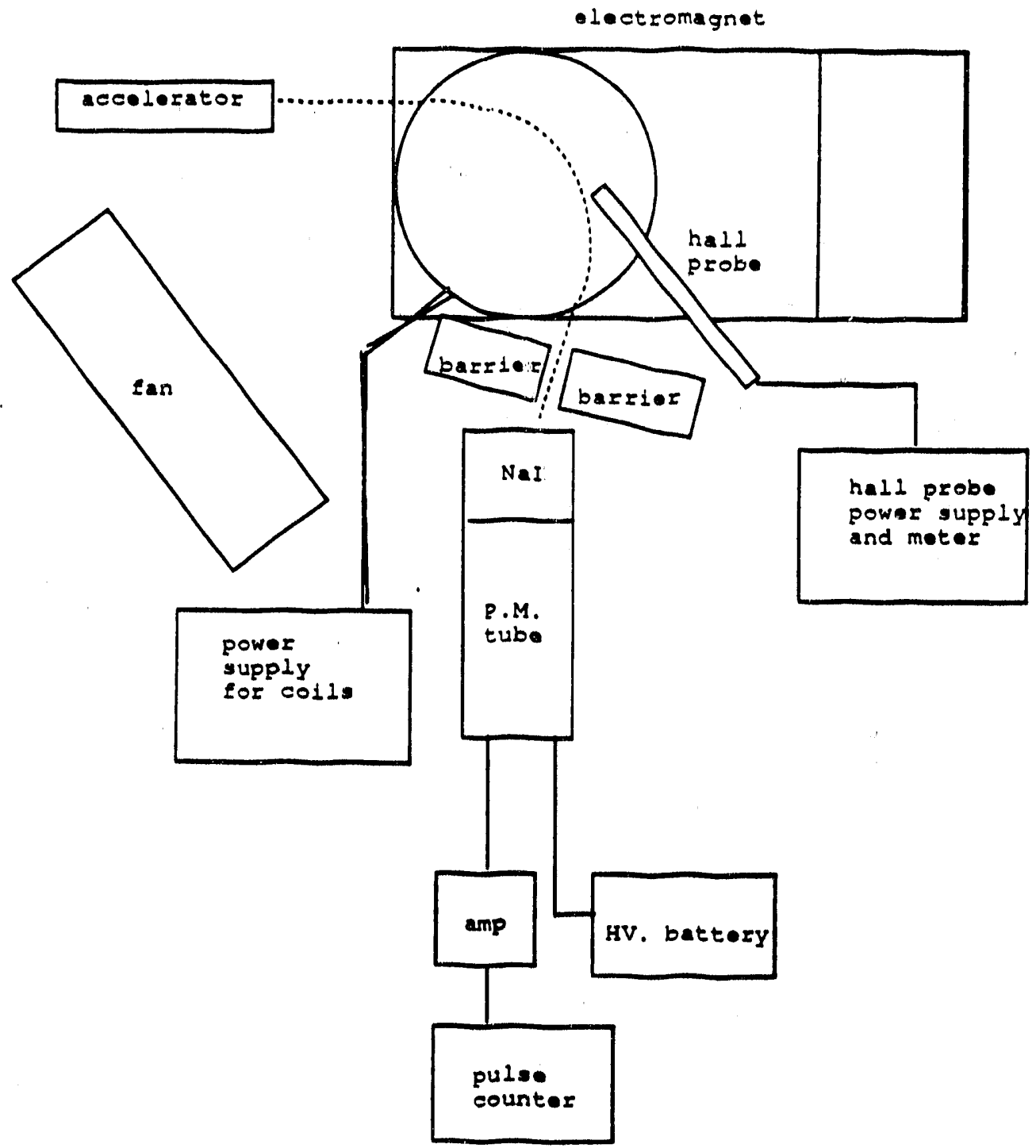


dark current electrons accelerated by the HGA. High power testing will commence upon installation of the energy analyzer.

\subsubsection{Electron Spectrometer}

A uniform magnetic field spectrometer was designed to measure any accelerated electrons from the dark current. The existing spectrometer design was found to be inadequate due to the severe space limitations near the HGA output window. A new design was made.

The spectrometer is made of low Carbon CMI-C electro-magnetic iron ASTMA 8.48-87. Fields of 1-1.2 Tesla can be reached without inhomogeneous magnetic field response. The device will operate in air and will allow for measurements of electrons at energies of up to $20 \mathrm{MeV}$. A schematic of the spectrometer and the associated experimental system components is shown in Fig. 18.

\section{Summary of Theoretical Research Progress}

This report summarizes technical progress and research accomplishments on high gradient acceleration and advanced accelerator concepts, supported under Task $\mathrm{C}$ of Grant No. DE- FG02-91ER40648 .by the Department of Energy, during the period June 1, 1991 through Feb. 1, 1992, A vigorous theoretical program has been pursued in critical problem areas related to advanced accelerator concepts, high gradient acceleration, and the basic equilibrium, stability, and radiation properties of intense charged particle beams. Broadly speaking, our research continues to make significant contributions in the following three major areas in which the group has established [3] significant expertise:

1. Investigations of physics issues related to particle acceleration including twobeam accelerators [4]-[8], asymmetric colliders [9] and cyclotron resonance laser (CRL) accelerators [10],[11].

2. Investigations of RF sources such as the free-electron lasers [12]-[17], cyclotron resonance masers [18]-[23], and relativistic magnetrons [24]-[28].

3. Studies of coherent structures in electron plasmas and beams ranging from a low-density, nonrelativistic, pure electron plasma column [26],[27],[29] to highdensity, relativistic, non-neutral electron flow in a high-voltage diode [24], [27].

The remainder of this report presents our recent theoretical and computational advances in these areas. Selected reprints and preprints of theoretical work are contained in Appendix F.

The theory effort has directly contributed to the photocathode design studies. This work is described in Sec. 1, and will not be presented separately here. 


\subsection{Two-Beam Accelerators and Colliders}

Significant advances have been made in the two-beam accelerator (TBA) concept. As described in the final report of our previous proposal [3], a major problem with the original version of the TBA was the strong sensitivity of the output $r$ phase to jitter in the system parameters. This difficulty was substantially alleviated by a change in the FEL design [4],[5], so that the rf power is now extracted at the end of each FEL section. This also solved the problem of power transport through the induction cells. A design study of TBA performance was conducted as an undergraduate thesis [8]. Further innovations were made when it was realized [6] that the FEL could couple to a series of short cavities, thus, essentially, making a standing wave FEL. This configuration is interesting not only in that it offers a realization of a method to extract and couple the rf power to the high gradient structure, but also in that it is new way to operate an FEL.

We have developed [7] an impedance model of the standing wave FEL. The formalism is quite general and can be used for the analysis of cumulative instabilities in standing-wave systems. The instabilities include transverse and longitudinal beam break-up in systems where the particle velocity in the cavity is curved. Applications of the formalism include a detailed comparison of the free electron laser and the relativistic klystron TBA. Further investigation will be required to see if this type of FEL has substantial advantages, in accelerator as well as non- accelerator applications, over conventional FEL oscillators where there is a single optical cavity which incorporates the entire FEL interaction region.

We have also investigated [9] the adiabatic disruption of asymmtric colliding beams. In this scheme, one beam is assumed to be much denser than the other and effectively becomes a lens for the second beam. The luminosity enhancement afforded by this asymmetric disruption process is estimated analytically. The concept is severely limited, however, by the kink instability which is characterized by a two-stream dispersion relation, and a constraint on the density of the less dense beam is found for which the kink instability is gradient stabilized.

\subsection{Cyclotron Resonance Laser Accelerators}

There has been considerable interest recently in laser accelerators [31] with the potential for achieving high accelerating gradient. One such system is the electron cyclotron resonance laser (CRL) accelerator [32] that makes use of the resonant interaction of a coherent electromagnetic wave with a beam of electrons gyrating in a (guide) magnetic field. An intriguing feature of the CRL mechanism is autoresonance [33],[34], that is, the synchronism between the electrons and laser field is self-sustained in the course of acceleration in an ideal situation so long as the system is initially synchronized. Although recent proof-of-principle experiments [35]-[37] have successfully demonstrated the acceleration of electrons to moderately relativistic energies using microwaves and the technique of tapering the guide field, there are severe limitations posed by laser field dispersion, synchrotron radiation losses, and the available strength of magnetic 
field in laboratories.

\subsubsection{Investigations of scaling properties in CRL accelerators}

The nonlinear interaction of a tenuous continuous electron beam with a traveling electromagnetic wave in the cyclotron resonance laser accelerator is analyzed (10]. using a single-particle model and multiparticle simulation. It is shown that there exist a maximum energy threshold and a maximum acceleration distance, which have not been reported in earlier studies. It is found that the maximum beam energy gin and acceleration distance obey certain scaling laws for optimized systems, that the maximum energy gain is limited primarily by the degree of wave dispersion and is almost independent of the wave amplitude. These scaling laws are important for future CRL accelerator experiments.

\subsubsection{Self-consistent modelling of high-current, high-efficiency CRL ac- celerators}

Design modelling of a high-current CRL accelerator in the microwave regime [11] is being performed using a three-dimensional self-consistent simulation code (CSPOT $[20],[23])$. The code takes into account the self-consistent evolution of the amplitude and phase of the driving wave due to the inverse cyclotron resonance interaction. The simulation includes the effects of $\mathrm{AC}$ space-charge in high-current beams, but does not include DC space-charge corrections to the electron orbits. The simulations show an acceleration efficiency of $\sim 80 \%$ and acceleration gradient of $\sim 2 \mathrm{MeV} / \mathrm{m}$. Ongoing research includes the evaluation of the CRL accelerator for the generation of $\mathrm{GeV}$ beams using intense laser pulses in the 1-10 micron wavelength range.

\subsection{Free-Electron Lasers}

The physics and technology of free-electron lasers (FELs) are intimately related to the accelerator community because FELs are important applications of particle accelerators and have the potential to power high-gradient if accelerators, such as in two-beam accelerators [4]-[6]. Under the auspices of this grant, several important aspects of the FEL have been investigated, including:.

(a) development of a three-dimensional axisymmetric (TDA) code for free-electron laser simulation [15],[16], and (b) experimental and theoretical investigations of nonlinear effects in a collective FEL [17].

\subsubsection{Development of the TDA code for FEL simulation}

The TDA simulation code [15],[16] has been developed and is widely used in the FEL community. Presently groups at MIT, CEBAF, LBL, UCLA, BNL and elsewhere are using the simulation to study the performance of various optical FEL systems. The TDA simulation solves the averaged FEL equations with fully three dimensional particle motion and the paraxial wave equation (with azimuthal symmetry assumed 
for the wave). Versions of TDA now include wiggler field errors and tapering schemes [38], multiple passes [39], and harmonics [40]. There is also a waveguide version of the simulation that can be used to investigate microwave and millimeter wave FELs [41]. Since the commencement of this grant, a fully three-dimensional version of TDA has been developed [42]. The radiation and space-charge fields are no longer assumed to be axisymmetric. The code can now accurately predict the diffractive losses due to wiggler errors and corrector fields. The new version of the simulation will be released to the user community within the next month. The next major simulation development will be a fully three -dimensional waveguide version including axial guide fields. The latter code should allow for more accurate modelling of the high efficiency FEL power source developed under Task B of this grant.

\subsubsection{Investigations of free-electron laser operation with a reversed guide field}

A theoretical study of the nonlinear performance of a FEL amplifier operating, with a reversed guide field, in the collective (Raman) regime is being conducted

The FEL generates up to $\sim 50 \mathrm{MW}$ of rf power at a frequency of $33.3 \mathrm{GHz}$ and an efficiency of $\sim 27 \%$. Power saturation, efficiency, and synchrotron oscillations are studied as a function of $\mathrm{rf}$ input power, electron beam energy, current, wiggler field amplitude, and axial distance with the helical wiggler. At present, the theoretical and simulation studies conducted at MIT and elsewhere have not successfully reproduced gain and saturation levels for both the reversed field and the group II orbits. Roughly, the reversed field behaves according to standard FEL theory, but the combined high growth rate and low saturated power is not consistent with linear theory or nonlinear simulations. (More precisely, simulations that reproduce the group II results do not reproduce the reverse field results). Our conclusion from this is that the present models lack some important physical aspect of the experiment, such as field misalignments or an unusual phase space distribution produced by the anode emittance selector. Further studies will be required to verify these speculations.

\subsection{Cyclotron Autoresonance Masers}

The cyclotron autoresonance maser (CARM) [43],[44] has received much interest recently as a potential source of high-power microwave and millimeter wavelength electromagnetic radiation. In particular, theoretical and experimental investigations are being conducted to examine the feasibility of the CARM amplifier as an RF source for future accelerators [45]. Under the auspices of this grant, we are continuing our extensive theoretical and computational investigations of the CARM:

Recent work has focused on (a) the influence of the RF space-charge waves generated by a gyro-phased electron beam. The influence of space charge on the bandwidth of the CARM and the efficiency has been examined using kinetic and single particle approaches. It is found that the space-charge enhances the growth rate at gyroresonance, but does not substantially reduce the peak growth rate of the instability. 
and (b) Using both kinetic and single-particle models to study the stability and equilibria of large amplitude waves. This work is similar to the sideband studies for the FEL and corresponds to a magnetized plasma analog to the BGK mode formalism.

\subsubsection{Development of the CSPOT code for CARM and CRL accelerator simulation}

As summarized previously, a three-dimensional, multimode, self-consistent code, CSPOT $[19],[20],[23]$ has been developed for the simulation of CARM amplifiers and CRL accelerators. The code can model a single TE or TM mode, Multiple TE and/or TM modes, cyclotron harmonics, magnetic field tapering, momentum and energy spread, waveguide losses, and various beam loading options. It has been benchmarked against a linear kinetic theory [19],[20], and has been found in good agreement with recent CARM amplifier experiments [21]. Space-charge terms have also been included in the CSPOT simulation and it being used to study the $17 \mathrm{GHz}$ CARM amplifier.

\subsection{Coherent Structures in Electron Plasmas and Beams}

It has been observed in various experiments and computer simulations that the ubiquitous appearance of coherent structures plays an important role in describing the dynamics of nonlinear systems governed by nonlinear partial differential equations. Coherent vortex structures, characterized by strong spatial and temporal correlations, occur in a variety of systems ranging from non-neutral plasmas with circulating electron flow [26],[27], to pure electron plasmas subject to diocotron instabilities in Penning trap configurations [26],[27], [29],[46],[47], to magnetrons [24],[48] and klystrons [49] for high-power microwave generation, to mention a few examples. Under the auspices of this grant, theoretical and computational investigations of coherent structures in electron beams and plasmas have been conducted. These have included: (a) analytical construction and computer simulation of vortex structures in low-density electron plasmas [29]-[30] and (b) investigations of the high current bunching in the relativistic klystron [50]

The development of nonneutral plasmas may, potentially, provide the accelerator community with a new high brightness source of electrons or positrons. In a Penning trap geometry, a nonneutral plasma column is confined magnetically in the radial direction and electrostatically in the axial direction. These plasmas exhibit a rich variety of nonlinear phenomena [27],[29],[30],[46],[47]. In the guiding-center approximation [26],[27], the underlying equations governing the dynamics are analogous to those of an ideal inviscid two-dimensional fluid. The plasma is then well-suited for studies of vortex dynamics and merging. We have constructed [29] a two-dimensional model of the plasma system . Our simulation allows for axial strips of the containing wall to be biased to a fixed potential (analogous to having a nonzero, curl-free, component to the velocity field), which, depending on the initial density profile, deforms the plasma equilibrium, induces a diocotron type motion, or generates a partial or total loss of the plasma. There are also plans [51] to conduct high resolution scat- 
tering experiments, related to fundamental tests of QED, using positrons confined in Penning traps and a $\mathrm{CW}$ electron beam. 


\section{References}

[1] Three-year research proposal entitled "Experimental and Theoretical Investigations of High Gradient Acceleration," DE-FG0291-ER40648 supported by the Department of Energy, Division of Nuclear and High Energy Physics.

[2] M. E. Conde and G. Bekefi, "Experimental Study of a $33.3 \mathrm{GHz}$ Free-Electron Laser Amplifier with a Reversed Axial Guide Magnetic Field," Phys. Rev. Lett $\underline{67}$ ,(1991)

[3] Final Report of a three-year research grant entitled "Advanced Accelerator Concepts", R.C. Davidson and J.S. Wurtele, Principal Investigators, supported by the Department of Energy, Division of Nuclear and High Energy Physics, 1988-1991.

[4] A.M. Sessler, E. Sternbach, J.S. Wurtele, W.M. Sharp, "A new version of a freeelectror laser two-beam accelerator," Nuclear Instruments and Methods B40/41, 1064 (1991).

[5] A.M. Sessler, D.H. Whittum, J.S. Wurtele, "Phase-control in the two-beam accelerator," Particle Accelerators 31, 69 (1990).

[6] A.M. Sessler, D.H. Whittum, J.S. Wurtele, W.M. Sharp, and M.A. Makowski, "A Standing-Wave Free-Electron Laser Two-Beam Accelerator," Nucl. Instr. and Meth. in Phys. Res., (1991).

[7] X.T. Yu, J.S. Wurtele and D. H. Whittum, "Impedance Formalism for Arbitrary Cumulative Instabilities," abstract submitted to APS spring meeting, manuscript in preparation.

[8] M. McCluskey, "Phase Sensitivity in the Two-Beam Accelerator," Bachelor of Science Thesis, MIT, 1991.

[9] "Adiabatic Disruption of Asymetric Colliding Beams," T. Katsouleas and J.S. Wurtele, Proc. of ICFA Workshop on Beam Dynamics, Los Angeles, 1991.

[10] C Chen, "Scaling Laws for the Cyclotron Resonance Laser Accelerator," Phys. Fluids B3, in press (1991).

[11] C. Chen, "Theory of High-Current Electron Cyclotron Resonance Laser Accelerators," manuscript in preparation (1991).

[12] C. Chen and R.C. Davidson, "Self-Field-Induced Chaoticity in the Electron Orbits in a Helical-Wiggler Free Electron Lasers with Axial Guide Field," Phys. Fluids B2, 171 (1990).

[13] C. Chen and R.C. Davidson, "Chaotic Electron Dynamics for Relativistic Electron Beam Propagation through a Planar-Wiggler Magnetic Field," Phys. Rev. A42, 5041 (1990). 
[14] C. Chen and R.C. Davidson, "Chaotic Particle Dynamics in Free Electron Lasers," Phys. Rev. A43, 5541 (1991).

[15] T.M. Tran and J.S. Wurtele, "TDA - A Three-Dimensional Axisymmetric Code for Free-Electron Laser (FEL) Simulation," Comp. Phys. Comm. 54, 263 (1989).

[16] T.M. Tran and J.S. Wurtele, "Free-Electron Laser Simulation Techniques," Phys. Rep. 195, 1 (1990).

[17] J.S. Wurtele, R. Chu, and J. Fajans, "Nonlinear Theory and Experiment of Collective Free-Electron Lasers," Phys. Fluids B2, 1626 (1990).

[18] C. Chen and J.S. Wurtele, "Efficiency Enhancement of Cyclotron Autoresonance Maser Amplifiers by Magnetic Field Tapering," Phys. Rev. A40, 489 (1989).

[19] C. Chen and J.S. Wurtele, "Multimode Interactions in Cyclotron Autoresonance Maser Amplifiers," Phys. Rev. Lett. 65, 3389 (1990).

[20] C. Chen and J.S. Wurtele, "Linear and Nonlinear Theory of Cyclotron Autoresonance Maser Amplifiers with Multiple Waveguide Modes," Phys. Fluids B3, 2133 (1991).

[21] A.C. DiRienzo, G. Bekef, C. Chen and J.S. Wurtele, "Experimental and Theoretical Studies of a $35 \mathrm{GHz}$ Cyclotron Autoresonance Maser Amplifier," Phys. Fluids B3, 1755 (1991).

[22] C. Chen, B.G. Danly, G. Shvets, and J.S. Wurtele, "Effect of Longitudinal SpaceCharge Waves of a Helical Relativistic Electron Beam on the Cyclotron Maser Instability," submitted for publication, Special Issue of IEEE Transaction on Plasma Science on High-Power Microwaves (1991).

[23] H.P. Freund and C. Chen, "Comparison of Gyro-Averajed and Non-GyroAveraged Nonlinear Analyses of Cyclotron Autoresonance Masers," submitted for publication, International Journal of Electronics (1991).

[24] H.-W. Chan, C. Chen and R.C. Davidson, "Computer Simulation of Relativistic Multiresonator Cylindrical Magnetrons," Appl. Phys. Lett. 57, 1271 (1990).

[25] C. Chen, H.-W. Chan, R.C. Davidson and G.L. Johnston, "Two-Dimensional Self-Consistent Simulation of the Relativistic Magnetron," Intense Microwave and Particle Beams, edited by H. Brandt, SPIE Vol. 1226, 100 (1990).

[26] R.C. Davidson, H.-W. Chan, C. Chen and S. Lund, "Large-Amplitude Coherent Structures in Nonneutral Plasmas with Circulating Electron Flow," AIP Conference Proceedings on Research Trends in Nonlinear and Relativistic Effects in Plasmas, in press (1990); MIT PFC Report PFC/JA-90-4. 
[27] R.C. Davidson, H.-W. Chan, C. Chen and S. Lund, "Equilibrium and Stability Properties of Intense Non-neutral Electron Flow," Rev. Mod. Phys. 63, 341 (1991).

[28] C. Chen, H.-W. Chan and R.C. Davidson, "Parametric Simulation Studies and Injection Phase Locking of Relativistic Magnetrons," Intense Microwave and Particle Beams II, edited by H. Brandt, SPIE Vol. 1407, 105 (1991).

[29] J. Notte, J. Fajans, T. Peurring, R. Chu, and J.S. Wurtele, "Asymmetric $\vec{E} \times$ $\vec{B}$ equilibria," abstract presented at the 1991 APS Division of Plasma Physics Meeting.

[30] "Collective Adiabatic Invariants in a Pure Electron Plasma," R. Chu, J.S. Wurtele, J. Notte and J. Fajans, abstract submitted to 1992 European Plasma Physics Conference.

[31] P.J. Channell, eds., Laser Acceleration of Particles, AIP Conf. Proc. No. 91, (American Institute of Physics, New York, 1982).

[32] P. Sprangle, L. Vlahos, and C.M. Tang, "A Cyclotron Resonance Laser Accelerator," IEEE Trans. Nuclear Sci. NS-30, 3177 (1983).

[33] C.S. Roberts and S.J. Buchsbaum, Phys. Rev. 135, 381 (1964).

[34] H.R. Jory and A.W. Trivelpiece, "Charged-Particle Motion in Large-Amplitude Electromagnetic Fields," J. Appl. Phys. 39, 3053 (1967).

[35] D.B. McDermott, D.S. Furuno, and N.C. Luhmann, Jr., "Production of Relativistic, Rotating Electron Beams by Gyroresonant RF Acceleration in a TE 111 Cavity," J. Appl. Phys. 58, 4501 (1985).

[36] K.S. Golovanevsky, "The Gyrac: A Proposed Gyro-Resonant Accelerator of Electrons," IEEE Trans. Plasma Sci. PS-10, 120 (1982).

[37] R. Shpitalnik, C. Cohen, F. Dothan, and L. Friedland, "Autoresonance Microwave Accelerator," J. Appl. Phys. 70, 1101 (1991).

[38] D.L. Goodman, et al., Proc. of the 13th International Free-Electron Laser Conference (1991).

[39] A.M. Sessler, et al., Proc. of the 13th International Free-Electron Laser Conference (1991).

[40] L.H. Yu (private communication).

[41] M. Ben-Ari and J.S. Wurtele (unpublished).

[42] P. Jha and J.S. Wurtele, "A Three-Dimensional FEL Simulation with SpaceCharge," in progress. 
[43] V.L. Bratman, N.S. Ginzburg, G.S. Nusinovich, M.I. Petelin, and P.S. Strelkov, "Relativistic Gyrotrons and Cyclotron Autoresonance Masers," Int. J. Electron. 51, 541 (1981).

[44] A.W. Fliflet, "Linear and Non-Linear Theory of the Doppler-shifted Cyclotron Resonance Maser Based on TE and TM Waveguide Modes," Int. J. Electron. 61. 1049 (1986).

[45] B.G. Danly, J.S. Wurtele, K.D. Pendergast, and R.J. Temkin, "CARM Driver for High Frequency RF Accelerators," Proceedings of the 1989 Particle Accelerator Conference (Chicago).

[46] K.S. Fine, C.F. Driscoll, and J.H. Malmberg, "Measurements of a nonlinear diocotron mode in pure electron plasmas," Phys. Rev. Lett. 63, 2232 (1989).

[47] K.S. Fine, C.F. Driscoll, J.H. Malmberg, and T.B. Mitchell, "Measurements of symmetric vortex merger," Phys. Rev. Lett. 67, 588 (1991).

[48] J. Benford, "Relativistic magnetrons," in High-Power Microwave Sources, eds., V. Granatstein and I. Alexeff (Artech House, Boston, Massachusetts, 1987), p. 309.

[49] Y.Y. Lau, M. Friedman, J. Krall, and V. Serlin, "Relativistic klystron amplifier driven by modulated intense relativistic electron beam," IEEE Trans. Plasma Sci. PS-18, 553 (1990).

[50] "Design Studies of a $3.3 \mathrm{GHz}$ Relativistic Klystron Amplifier," C. Chen, P. Catravas, and G. Bekefi, to be published in Proc. of SPIE Conf. Vol. 1629 (1992).

[51] T. Cowan and J. Fajans, private communication. 
Appendix A. Specification of the Laser Parameters 
Picosecond UV Laser System for

High Gradient Accelerator Experiment

January 23, 1992

$\begin{array}{lll}\text { Please contact: } & \text { Seth Trotz } & \text { S.C. Chen } \\ & \text { MIT } & \text { MIT } \\ \text { NW 16-180 } & \text { NW 16-176 } \\ \text { Cambridge, MA 02139 } & \text { or } \quad \begin{array}{l}\text { Cambridge, MA 02139 } \\ (617) 253-8751\end{array} & \begin{array}{l}(617) 253-0833 \\ \text { Fax (617) 253-6078 }\end{array} \\ & \text { Fax (617) 253-6078 }\end{array}$

Thank you,

\section{I.1 Introduction}

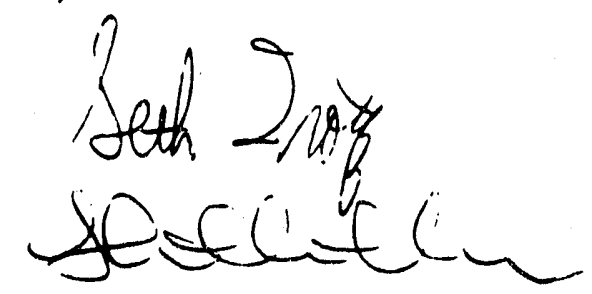

The Plasma Fusion Center at M.I.T. currently has a program to study the possibility of particle acceleration at high field gradients. Our initial experiment has been designed as a $17 \mathrm{GHz}$ photocathode RF gun. The RF gun is a microwave cavity containing a cathode. The cavity is excited by a $17 \mathrm{GHz}$ microwave pulse of duration $30-50 \mathrm{~ns}$ and power of 2-5 MW. The short pulse laser releases electrons from the cathode which are then accelerated by the microwave field. The laser pulse must be applied at an optimal phase point of the $17 \mathrm{GHz}$ microwave ( $\mathrm{RF}^{*}$ ) field. This requirement places stringent tolerances on the laser pulse length and timing jitter. The high frequency of the gun necessitates a short pulse laser with extremely low timing jitter.

It has been determined that the laser system will be purchased in one of two options. The first and preferred option is to purchase a complete laser system from a single vendor. The second option consists of buying the laser system as a collection of components instead of as a complete system. The second option will be chosen if the first option proves prohibitively expensive. A vendor may choose to return a bid for either or both options.

\footnotetext{
- Note : $17 \mathrm{GHz}$ is a microwave frequency but it is often referred to as an "RF" frequency in the present application.
} 
Option I

Each vendor entering a bid for option I is requested to present a complete design of the laser system including all necessary components. Under this option, all components will be bought from the vendor whose bid is chosen. Cost and delivery information are also requested.

\section{Option II}

If a vendor enters a bid for the second option, the following information will be needed. The vendor is requested to present as complete a design of the laser system as possible including all necessary components. Cost, delivery, and technical information are requested on those parts sold by the vendor. We also ask that recommendations of other sources be made for all other components necessary for the vendor's design. If option II is selected, MIT will select a final system design, most likely based on designs suggested by vendors. MIT will request a conference to review the system design. Vendors must be willing to attend such a conference. They must also be willing to review the final system design and verify its suitability to the best of their knowledge. Vendors must also be willing to assist MIT while MIT integrates the final system and conducts system tests. Since the laser system will be integrated in the field, after-the-sale advice and technical support will be mandatory.

Section 1.2 indicates a laser system design which MIT believes is promising for meeting the specifications outlined in Sections II and III. Section I.2 is provided as an illustrative example. Vendors may propose alternate designs or use the design of section I.2. 


\section{I.2 Illustrative Example}

The functional blocks of a possible laser system suitable for our application are shown in figure 1. Vendors are encouraged to present alternative approaches if they are attractive. A CW modelocked laser oscillator is used to seed subsequent amplifier(s) to achieve the required energy per pulse. The choice of modelock frequency will be made as follows :

1. MIT will cold test the RF cavity to determine the exact RF frequency which will lie near $17.136 \mathrm{GHz}$. Label it $17 . x \mathrm{GHz}$.

2. MIT will provide the $17 . \times \mathrm{GHz}$ number to the vendor.

3. The vendor will choose a subharmonic of $17 . x \mathrm{GHz}$ to be the modelock frequency of the laser.

4. MIT will supply the actual modelock signal to the vendor's laser.

In the following example, $59.5 \mathrm{MHz}$ is used as the modelock frequency. There is nothing special about this frequency. The modelock frequency may be any subharmonic of the $17 . x \mathrm{GHz}$ signal from $48-238$ $\mathrm{MHz}$ but will preferably be below $100 \mathrm{MHz}$ where the subharmonics are more densely packed. A 48-238 $\mathrm{MHz}$ modelock frequency will give a ratio of frequencies between and 357 and 72. The vendor should choose a modelock frequency that is consistent with the laser cavity design. Also, for our application it is highly desirable to have a modelock frequency which is tunable within $\pm 0.3 \%$ range. The vendor should provide access to the cavity length tuning and specify the available range.

The laser oscillator may have to be actively stabilized to reduce phase jitter. External chirping/ stretching and compression elements may be required to obtain the picosecond pulse length specification. Frequency conversion into UV is required.

The manner in which the laser system is to be integrated into the MIT RF gun program is illustrated in figure 2. The amplifier must be able to respond to an external trigger signal on a single shot basis and also in a repetition mode of $0-10 \mathrm{~Hz}$. The laser system should accept a $59.5 \mathrm{MHz}$ reference input as the seed for the modelock driver.

\subsection{Components of Example System}

1. Timing Stabilization

2. Laser Oscillator

3. Optical Fiber and related optics

4. Laser Amplifier

5. Grating Compression and Related Optics (if required)

6. Frequency Conversion Crystals

7. Other Optics (as required)

(if required) 
Fig 1 : Example Laser System

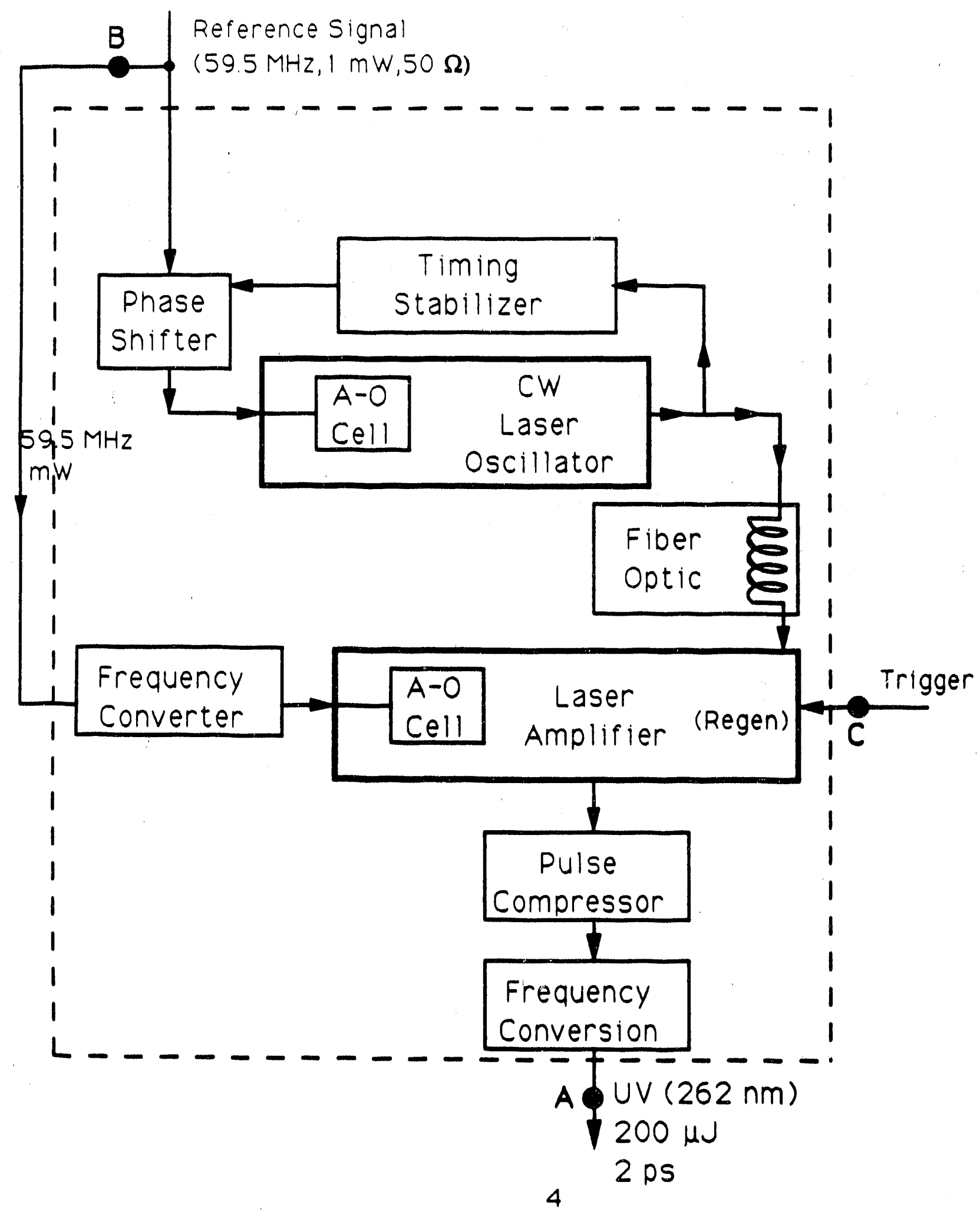


Fig 2:Timing Between Laser System \& CARM

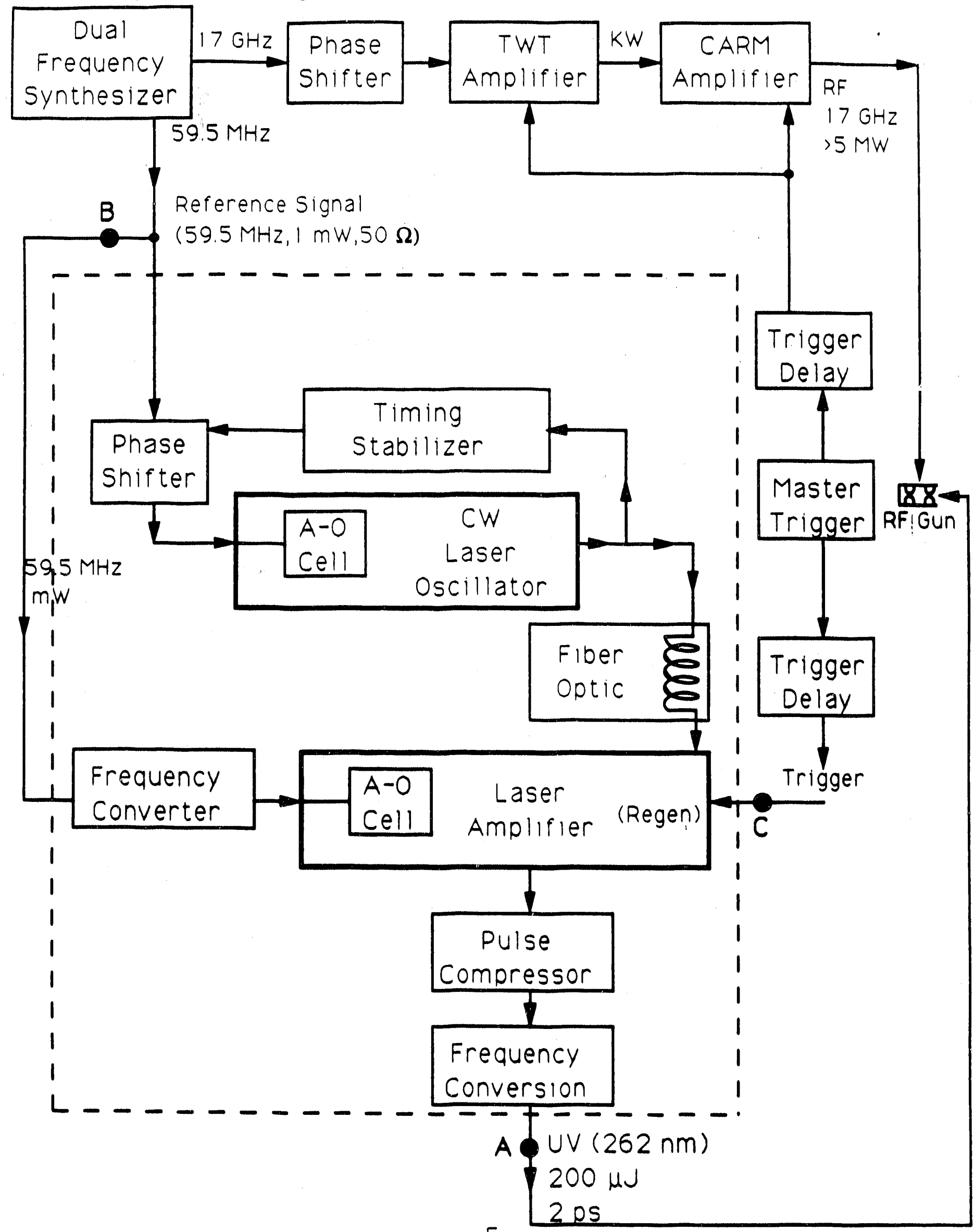


Fig 3:Optimal Timing Diagram in the RF Gun

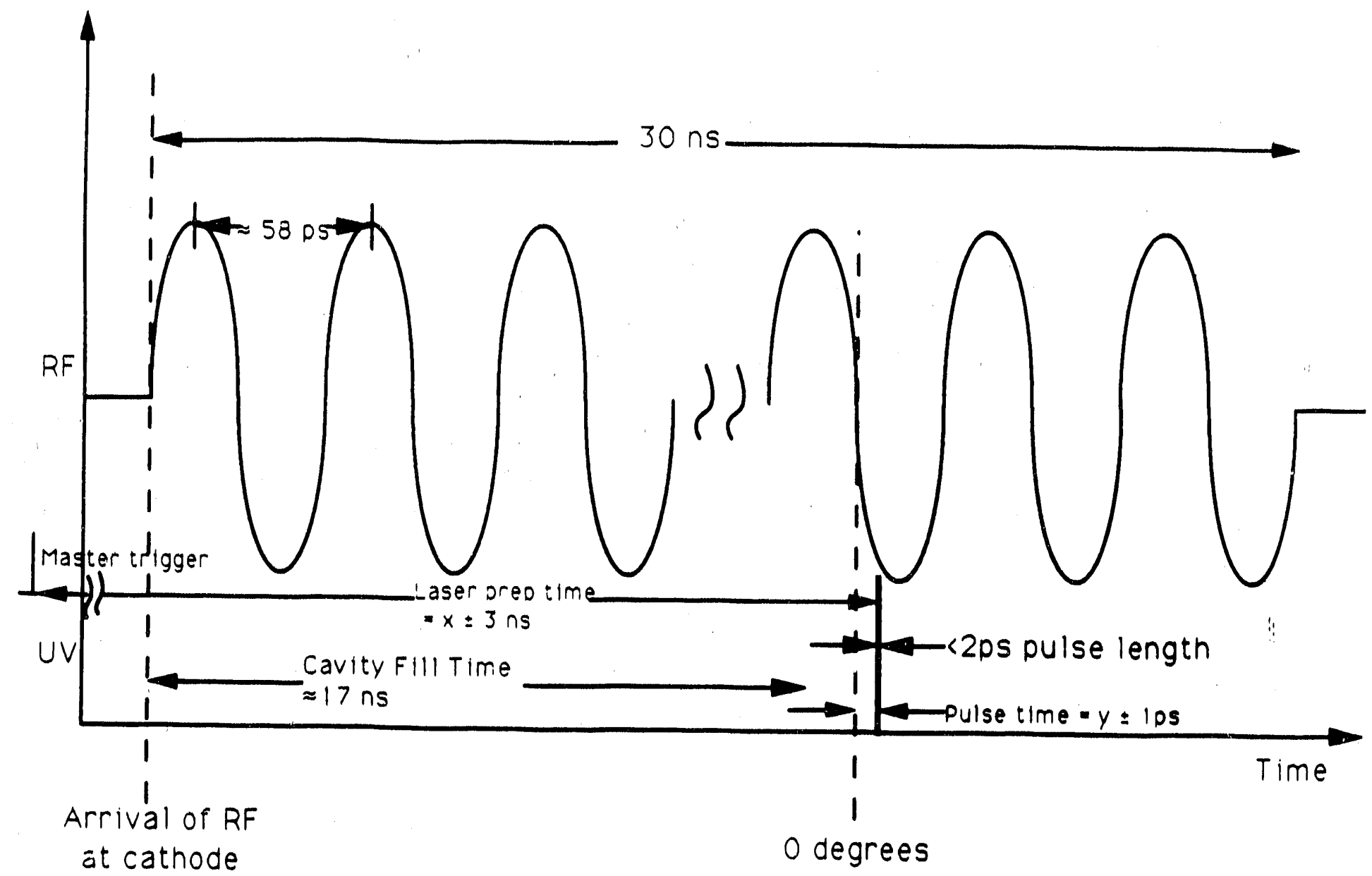

With a proper and stable time delay between the firing of the CARM amplifier and the firing of the laser amplifier, we expect the laser pulse to arrive at the cathode surface when the RF gun cavity is fllled with the microwaves from the CARM. Figure 3 shows the optimal timing diagram. The upper trace shows the 30 ns RF pulse oscillating at about 17.136 $\mathrm{GHz}$ (58 ps perlod) and filling up the cavity after about $17 \mathrm{~ns}$. The laser amplifier should be fired in such a way so that the output laser pulse (the lower trace in figure 3) reaches the fllled cavity at the optimal microwave phase. It is of critical importance to have the generation of photoelectrons synchronized with the microwave phase to within I ps to obtain proper acceleration. 
II. Performance Specifications for the Laser System

\section{II.1 Parameters}

Wavelength after frequency conversion $220-280^{1} \mathrm{~nm}$

Repetition rate of the laser system Single pulse and $0-10 \mathrm{~Hz}$ (adjustable)

Final output energy (per pulse) in UV $0-200 \quad \mu \quad \mu \quad$ (adjustable)

Energy output fluctuation (pulse-pulse) $\leq \pm 10 \quad \%$

Pulse length (autocorrelation measured) $<2$ ps

Phase jitter (Fig 1. A and B) $<1 \quad$ ps

Timing jitter (Fig 1. A and C) $<3 \quad$ n s

Polarization $>99 \quad \%$

Beam divergence Near diffraction limit

Beam pointing error $<10 \quad$ Hrad

Mode-lock frequency ${ }^{2} \quad 48-238 \quad \mathrm{MHz} \quad$ (adjustable)

$( \pm 0.3 \%)$

1 We specify here the acceptable range of frequencies. The wavelength does not need to be tunable.

2 The mode lock frequency should be chosen to be a subhamonic of our RF frequency at about $17.136 \mathrm{GHz}$. 


\section{II.2 Additional Notes on Specification}

1. Single laser pulse: Only a single pulse should arrive at the RF gun for each trigger signal. Remaining pulses should be discarded in a way that will not damage the oscillator, amplifier, or other elements of the system.

2. Jitter: Phase jitter is defined to be the jitter between the laser pulse arrival time (point $\mathrm{A}$ in Figure 2) relative to the "reference signal" at 59.5 $\mathrm{MHz}$ (point $\mathrm{B}$ in Figure 2). Timing jitter is defined to be the jitter between the "master trigger signal" and the laser pulse arrival time (point $A$ in figure 2). This is the so-called, pulse to pulse jitter. Both the phase and timing jitter are stringent requirements of the system. We emphasize here the need to consider and minimize all possible contributions to these factors which must be $<1$ ps (rms) and $<3$ ns respectively. For example, thermal expansion of fiber optic cable, fluctuations in the laser amplifier, and variability in flash lamp buildup time may create phase and timing jitter. Please describe a method for measuring the phase jitter.

3. Beam characterization: The vendor should provide figures on the laser beam size, power density, power, and pulse length at each point in the system. MIT will be adding hardware to the system to measure the final output laser pulse length and profile. Advice on optimal methods for performing these measurements is requested with the design specification.

4. Uparadeability: The laser system should be ufgradeable to $2 \mathrm{~mJ}$ per pulse with the insertion of additional amplification stages. 


\section{Qperational and Physical Requirements}

III.1 Monitoring control, and safety provisions- The laser must be adequately instrumented to permit both local and remote control. Appropriate safety interlocks and alarms should be provided.

III.2 Laser Trigger - The laser system must respond to an external signal to trigger the laser. The input trigger should be able to trigger the system at a repetition rate of $0-10 \mathrm{~Hz}$ and single shot.

III.3 Laser Output Energy - The laser system must permit adjustment of the final output energy from 0 to $200 \mu \mathrm{J}$ per pulse.

III.4 Timing Signal - The laser modelocking signal will be supplied by a Jual frequency synthesizer by MIT. For factory testing purposes, vendors will need to supply an external $1 \mathrm{~mW}$ signal to their laser oscillator which will be replaced at MIT by our own signal.

III.5 Modelocking Frequency Tuning - The modelocking frequency of the iaser oscillator should be tunable within a range of $\pm 0.3 \%$. The vendor should specify the available tuning range. This tunability will be necessary to match the modelock frequency to the actual, laboratory tested, resonant frequency of the microwave cavity which will be nearly but not exactly equal to $17.136 \mathrm{GHz}$.

III.6 Physical Size - It is important that the laser system have a minimal footprint due to limited floorspace for the experiment. Please specify the physical size of the system.

III.7 Operating Environment - Temperature, humidity, and power requirements should be specified.

III.8 Documentation - All necessary documentation, schematics, and operating manuals should be specified with system components.

III.9 Operating Conditions and Cost - The vendor should provide a specification of further operating conditions, costs, warranties, and maintenance schedules. 
IV. Teste - Due to the different approaches involved in options one and two, the testing requirements of the laser system will be described in a case by case manner.

\section{IV.A Option One Testing}

IV.A.1 Eactory Tests - The entire laser system should be tested by the vendor at the factory to ensure compliance with the specifications and requirements described in sections II and III.

IV.A.2 Final acceptance tests - Final acceptance tests will be completed after delivery of the complete system to MIT. Final payment will be made after the final acceptance tests are completed.

\section{IV.B Qption Two Testing}

IV.B.1 Eactory Tests - All individual components should be factory tested to meet their own specifications.

IV.B.2 Final acceptance tests - Since the system is being put together at MIT, final acceptance tests will be completed after the system has been integrated. During the integration and testing period, technical support will be vital fiom all vendors supplying components of the system. Final payment will be made after the final acceptance tests are completed. 


\section{Source List}

1. Coherent

45 Myrtle Street \#23

Boston, MA 02114

Attn: Steve McDonald

(617) 248-1834

Fax (617) 248-1835

3. Continuum

152 Dartmouth Street.

Marlboro, MA 01752

Attn: David Kemp

(508) $\quad 624-4454$

Fax (508) 624-4453

4. Lightwave Electronics

1161 San Antonio Road

Mountain View, CA 94043

Attn: Tim Gray

(415) $962-0755$

Fax (415) 962-1661

6. Quantronix Corporation

49 Wireless Boulevard

P.O. Box 9014

Smithtown, NY 11787-9014

Attn: John S. MacKay

(516) 273-6900

Fax (516) 273-6958
2. Positive Light Incorporated

6116 Highway 9 Suite 2B

Felton, CA 95018

Attn : Ron Olson

(408) 335-2288

Fax (Unknown)

3150 Central Expressway

Santa Clara, CA 95051

or Attn: John Davis

(408) $727-3240$

Fax (408) $727-3550$

5. Medox

1919 Green Rd, \# A128

Ann Arbor, MI 48105

Attn: P. Bado

(313) $930-0365$

Fax (313) 930-0371

7. Spectra Physics

251 Butman Rd.

Lowell, MA 01852

Attn: Jerry Romanek

(508) 459-3037

Fax (508) 459.3092 
Appendix B. Phase Locking and Synchronization 


\section{MODELOCK.OUT}

APPENDIX II

AVAILABLE HARMONIC NUMBERS AND MODELOCK FREQUENCIES EOR THE MIT $17.136 \mathrm{GHz}$ (*) RE GUN

(*) The exact central RF frequency will be provided later by MIT which maybe as much as 5 of of the ideal $17.136 \mathrm{GHz}$. The chosen vendor may wait for the exact frequency or use a modelocker with the 5 t tuning range.

\begin{tabular}{ccccc}
$\begin{array}{c}\text { Harmonic } \\
\text { Number }\end{array}$ & $\begin{array}{c}\text { Modelock } \\
\text { Erequency } \\
\text { (MHz) }\end{array}$ & $\begin{array}{c}2 \\
\text { power }\end{array}$ & $\begin{array}{c}3 \\
\text { power }\end{array}$ & $\begin{array}{c}5 \\
\text { power }\end{array}$ \\
\hline & & & &
\end{tabular}

\begin{tabular}{|c|c|c|c|c|}
\hline 64 & 267.75 & 6 & 0 & 0 \\
\hline 72 & 238 & 3 & 2 & 0 \\
\hline 75 & 228.48 & 0 & 1 & 2 \\
\hline 80 & 214.2 & 4 & 0 & 1 \\
\hline 81 & 211.5556 & 0 & 4 & 0 \\
\hline 90 & 190.4 & 1 & 2 & 1 \\
\hline 96 & 178.5 & 5 & 1 & 0 \\
\hline 100 & 171.36 & 2 & 0 & 2 \\
\hline 108 & 158.6667 & 2 & 3 & 0 \\
\hline 120 & 142.8 & 3 & 1 & 1 \\
\hline 125 & 137.088 & 0 & 0 & 3 \\
\hline 128 & 133.875 & 7 & 0 & 0 \\
\hline 135 & 126.9333 & 0 & 3 & 1 \\
\hline 144 & 119 & 4 & 2 & 0 \\
\hline 150 & 114.24 & 1 & 1 & 2 \\
\hline 160 & 107.1 & 5 & 0 & 1 \\
\hline 162 & 105.7778 & 1 & 4 & 0 \\
\hline 180 & 95.2 & 2 & 2 & 1 \\
\hline 192 & 89.25 & 6 & 1 & 0 \\
\hline 200 & 85.68 & 3 & 0 & 2 \\
\hline 216 & 79.33334 & 3 & 3 & 0 \\
\hline 225 & 76.16 & 0 & 2 & 2 \\
\hline 240 & 71.4 & 4 & 1 & 1 \\
\hline 243 & 70.51852 & 0 & 5 & 0 \\
\hline 250 & 68.544 & 1 & 0 & 3 \\
\hline 256 & 66.9375 & 8 & 0 & 0 \\
\hline 270 & 63.46667 & 1 & 3 & 1 \\
\hline 288 & 59.5 & 5 & 2 & 0 \\
\hline 300 & 57.12 & 2 & 1 & 2 \\
\hline 320 & 53.55 & 6 & 0 & 1 \\
\hline 324 & 52.88889 & 2 & 4 & 0 \\
\hline 360 & 47.6 & 3 & 2 & 1 \\
\hline 375 & 45.696 & 0 & 1 & 3 \\
\hline 384 & 44.625 & 7 & 1 & 0 \\
\hline 400 & 42.84 & 4 & 0 & 2 \\
\hline 405 & 42.31111 & 0 & 4 & 1 \\
\hline 432 & 39.66667 & 4 & 3 & 0 \\
\hline 450 & 38.08 & 1 & 2 & 2 \\
\hline 480 & 35.7 & 5 & 1 & 1 \\
\hline 486 & $\overline{3} 5 . \overline{2} \overline{5} \overline{\mathrm{C}} \overline{\mathrm{C}} \overline{\mathrm{C}}$ & $I$ & 5 & 0 \\
\hline
\end{tabular}


Appendix C. Cavity Design and Vacuum System 

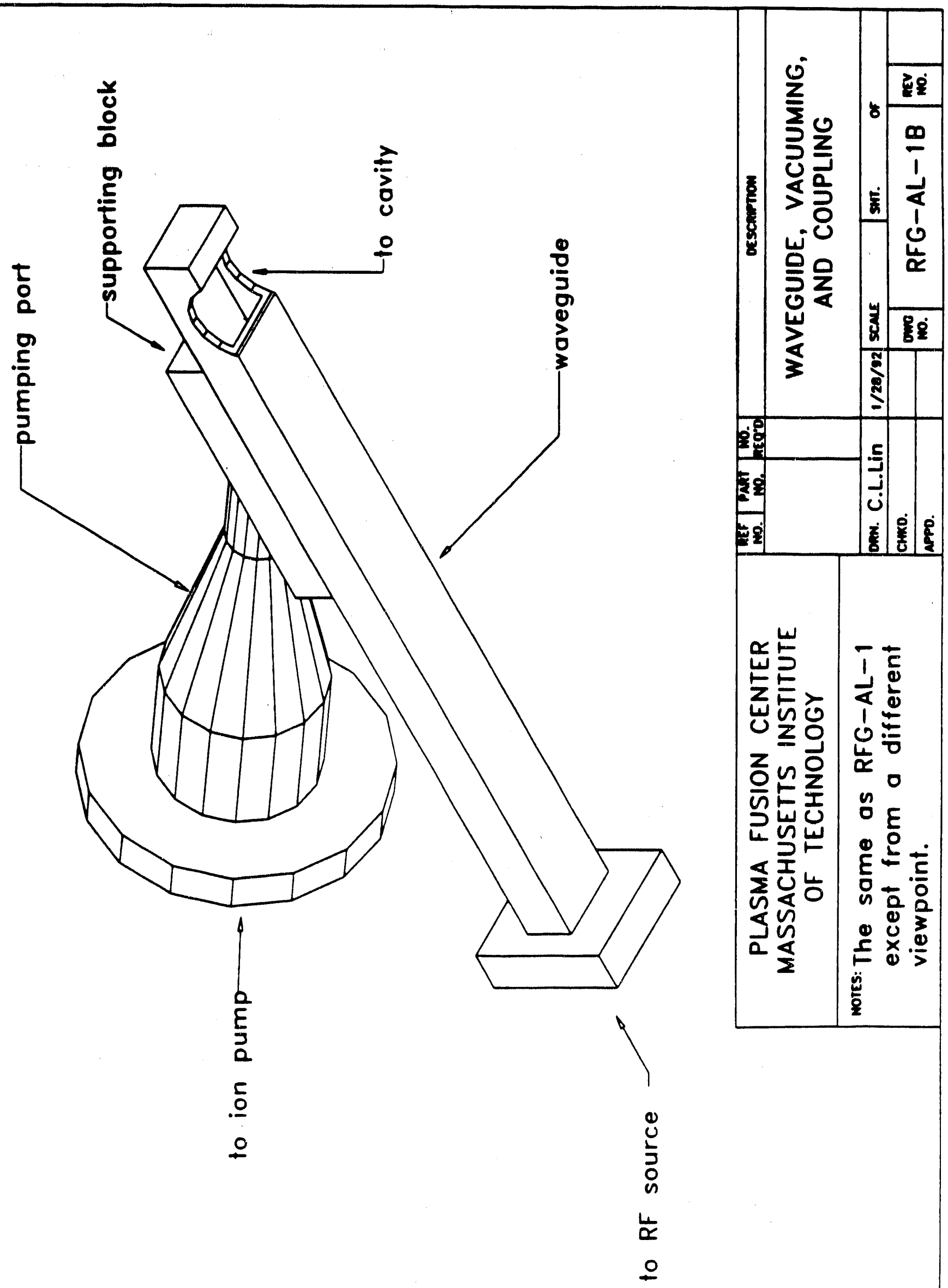


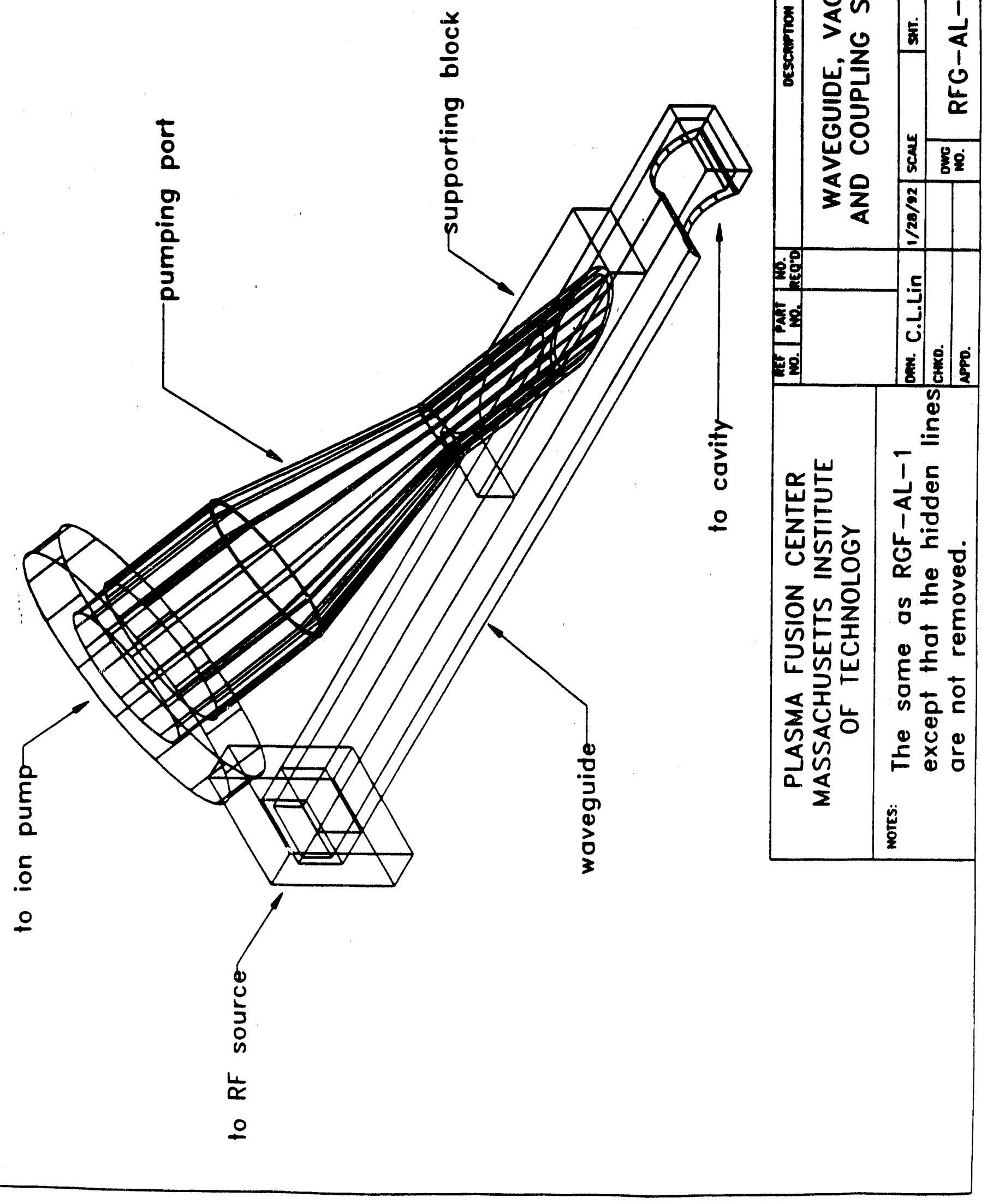




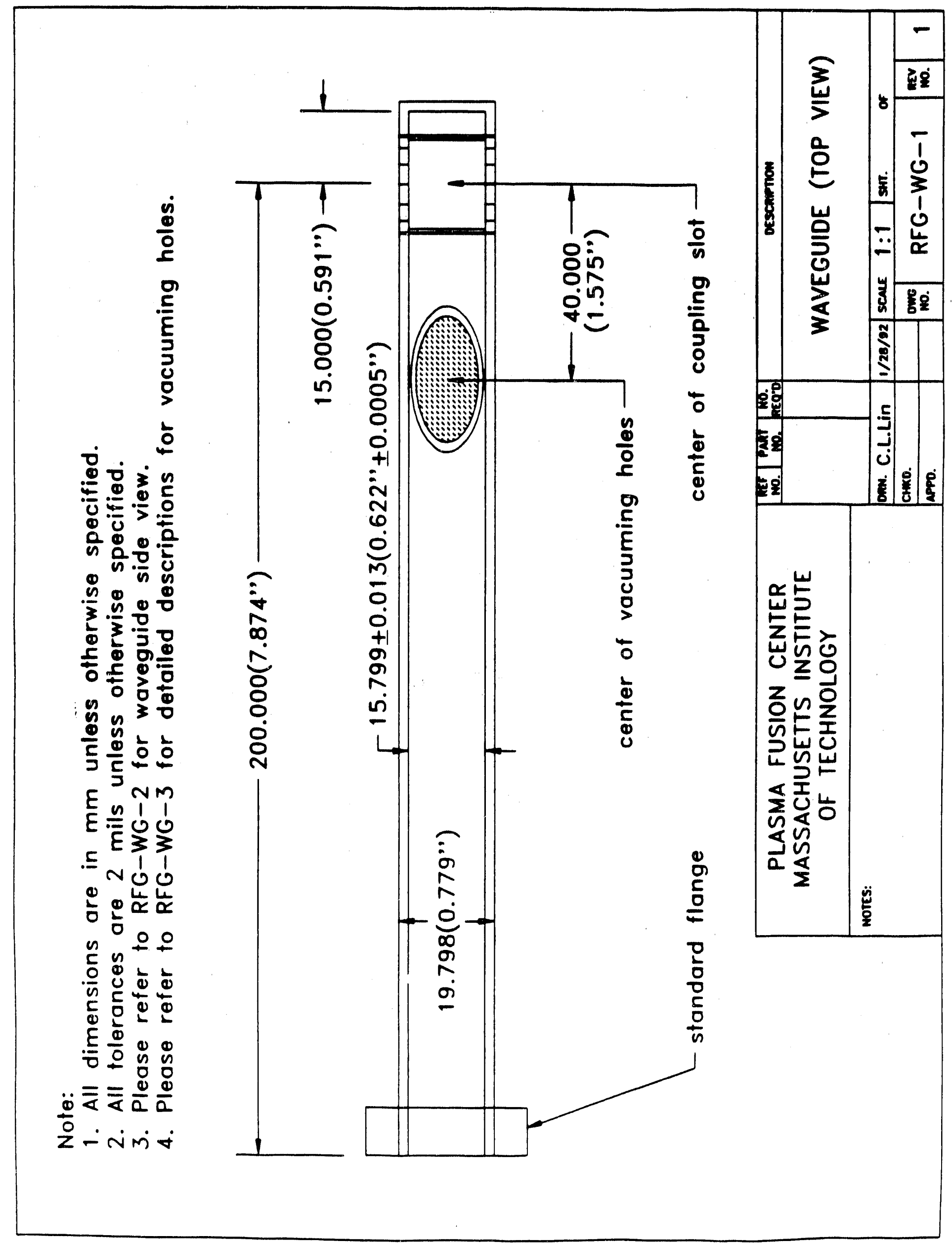




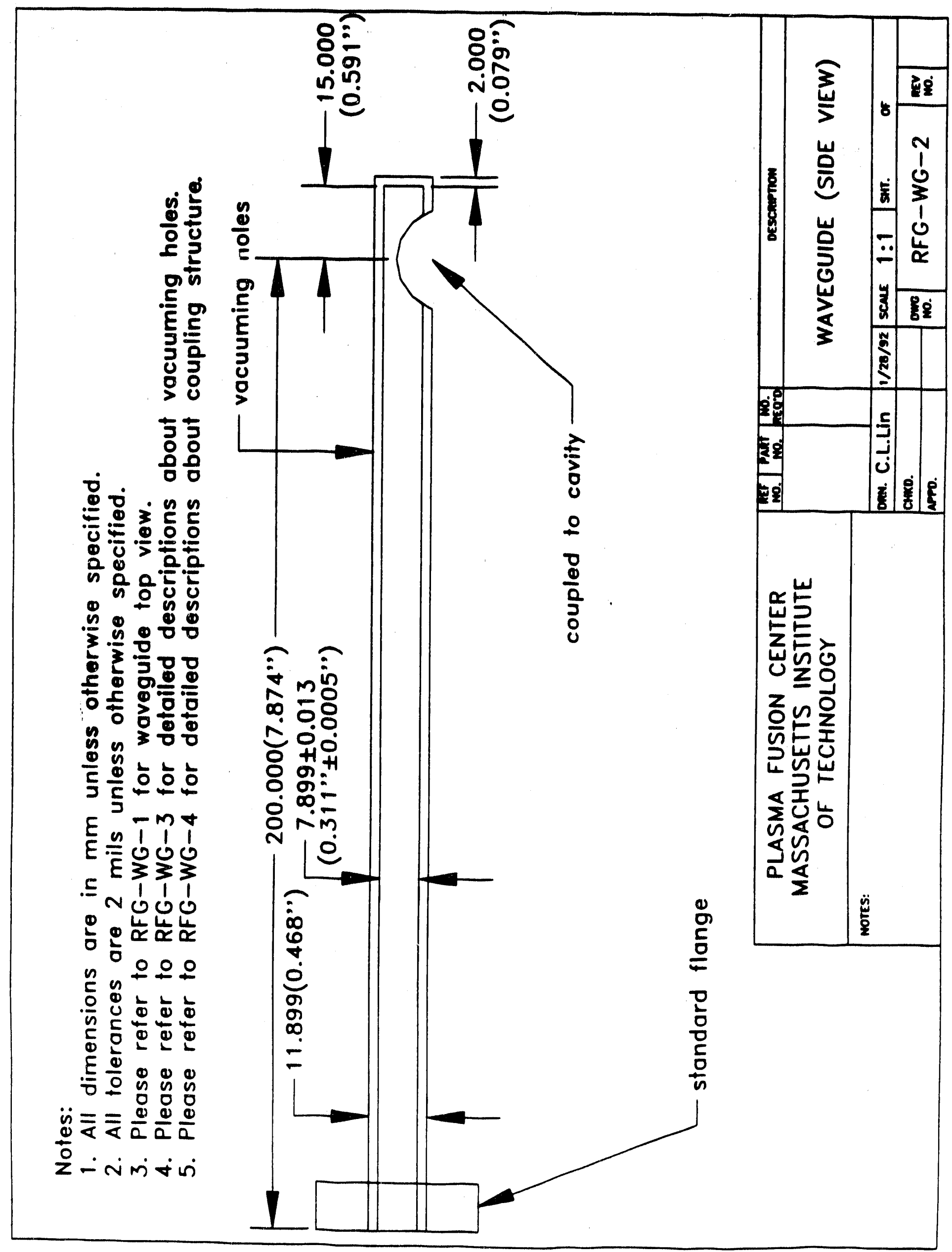




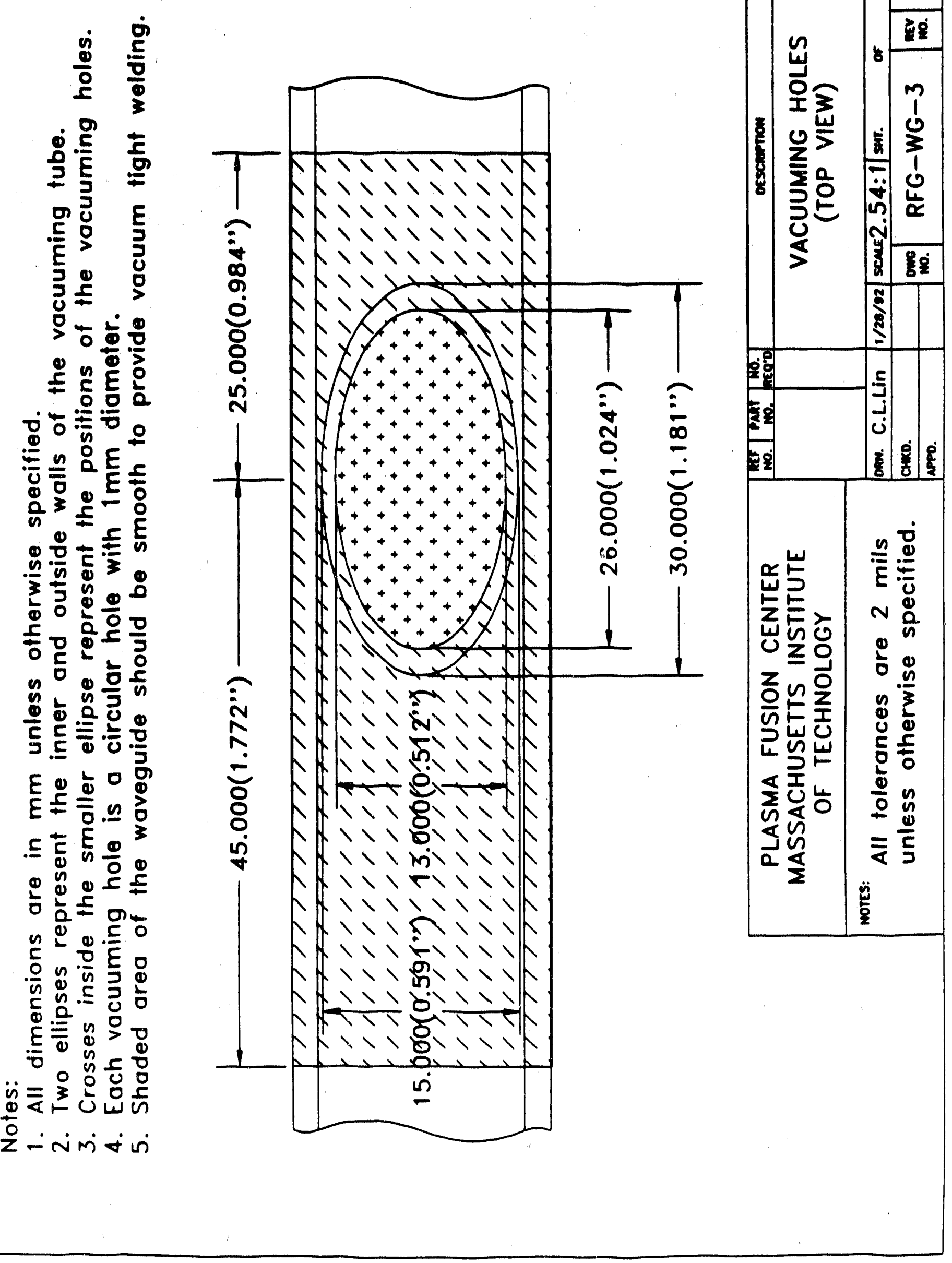


Notes:

1. All dimensions are in $\mathrm{mm}$ unless otherwise specified.

2. All tolerances are 2 mils unless otherwise specified.

3. The circular section should be smooth to provide vacuum tight welding.

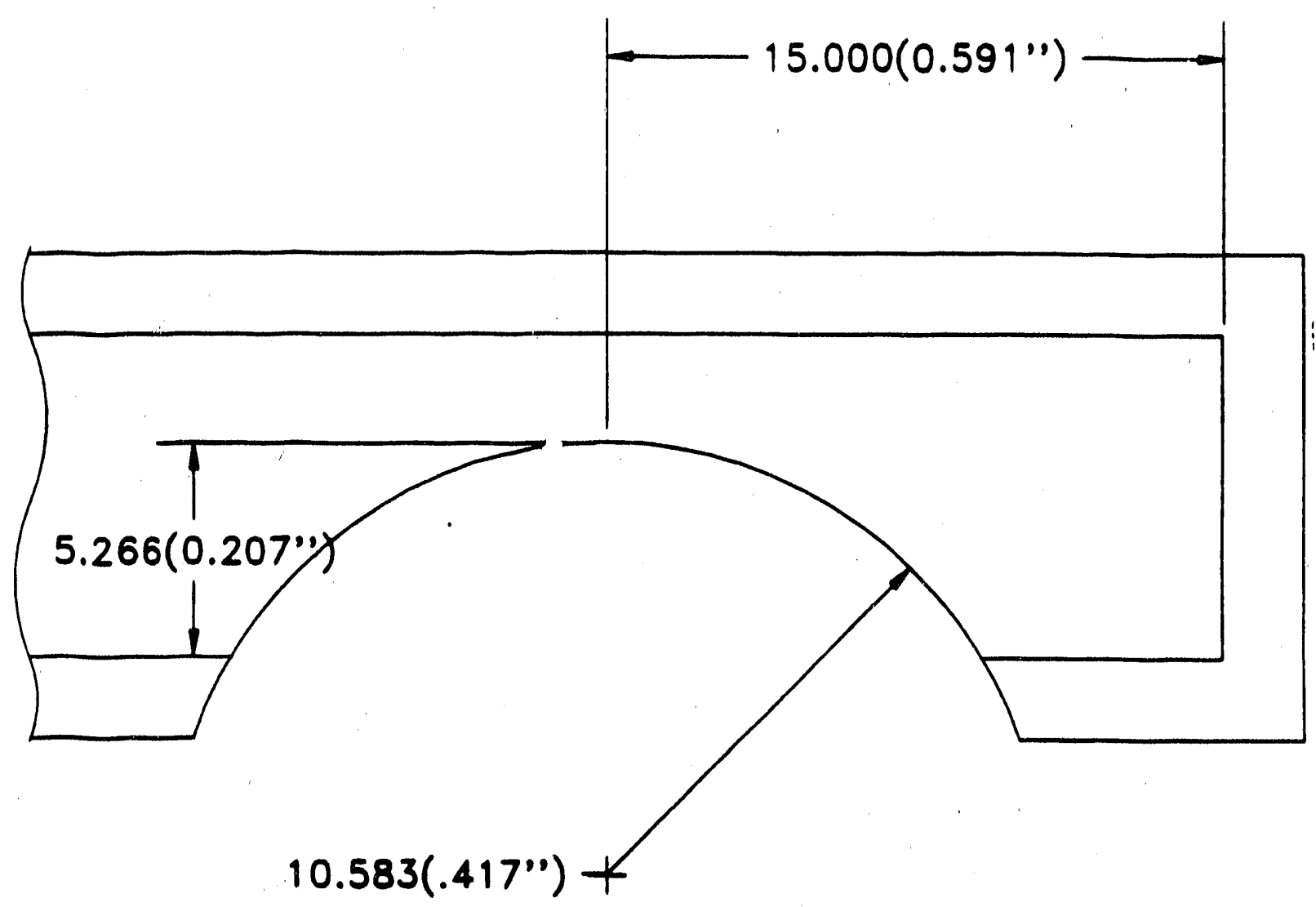

PLASMA FUSION CENTER MASSACHUSETTS INSTITUTE OF TECHNOLOGY

\begin{tabular}{|c|c|c|c|c|c|c|}
\hline${ }_{m}\left[x_{1}^{\infty}\right.$ & \multicolumn{6}{|c|}{ orschumion } \\
\hline & \multicolumn{6}{|c|}{ COUPLING STRUCTURE } \\
\hline onv. C.L.Lin & $1 / 20 / 102$ & & $.08: 1$ & sin. & & \\
\hline$\frac{\text { ento. }}{\text { anpo. }}$ & & 然o. & RFG- & $-W G-4$ & act & 1 \\
\hline
\end{tabular}




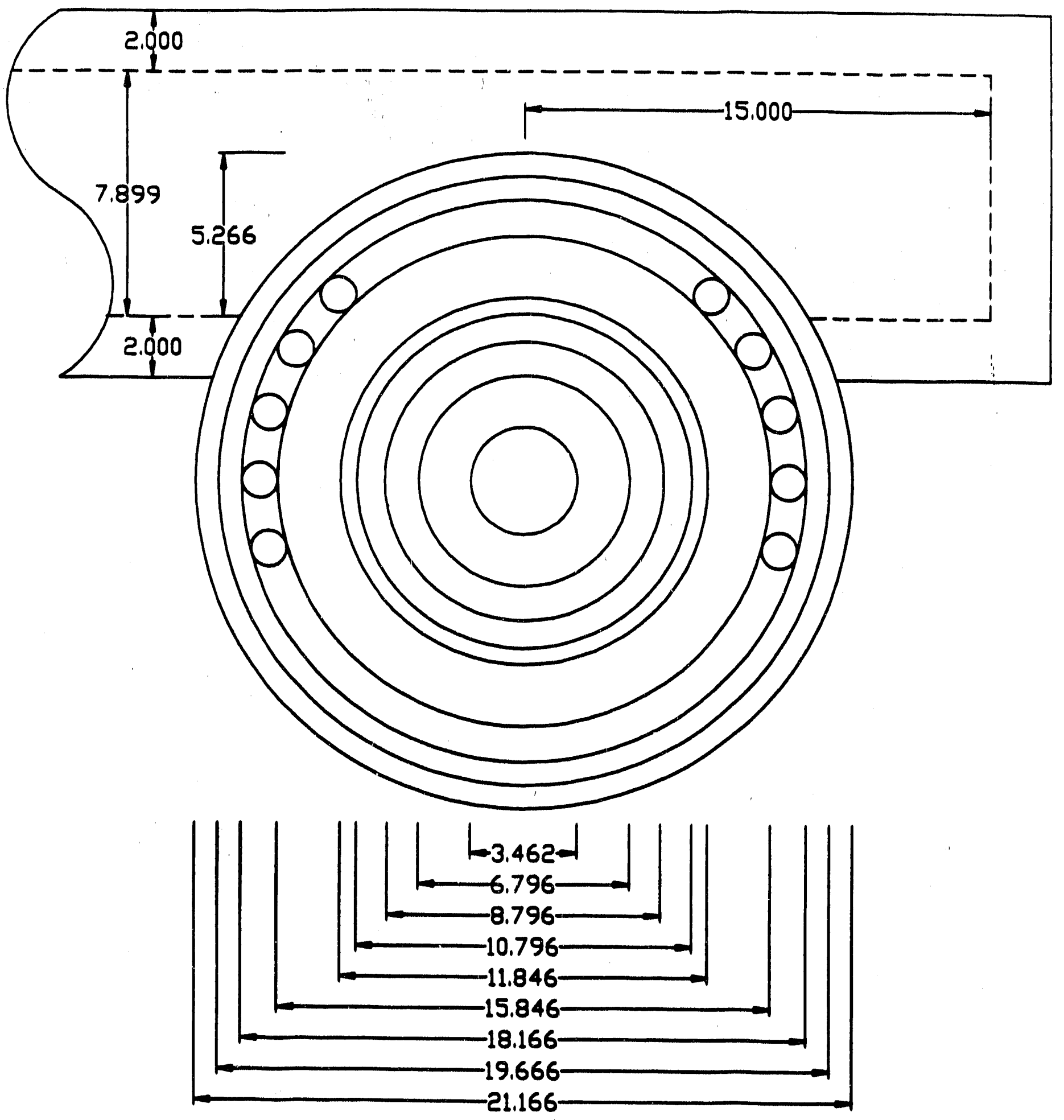

front view 


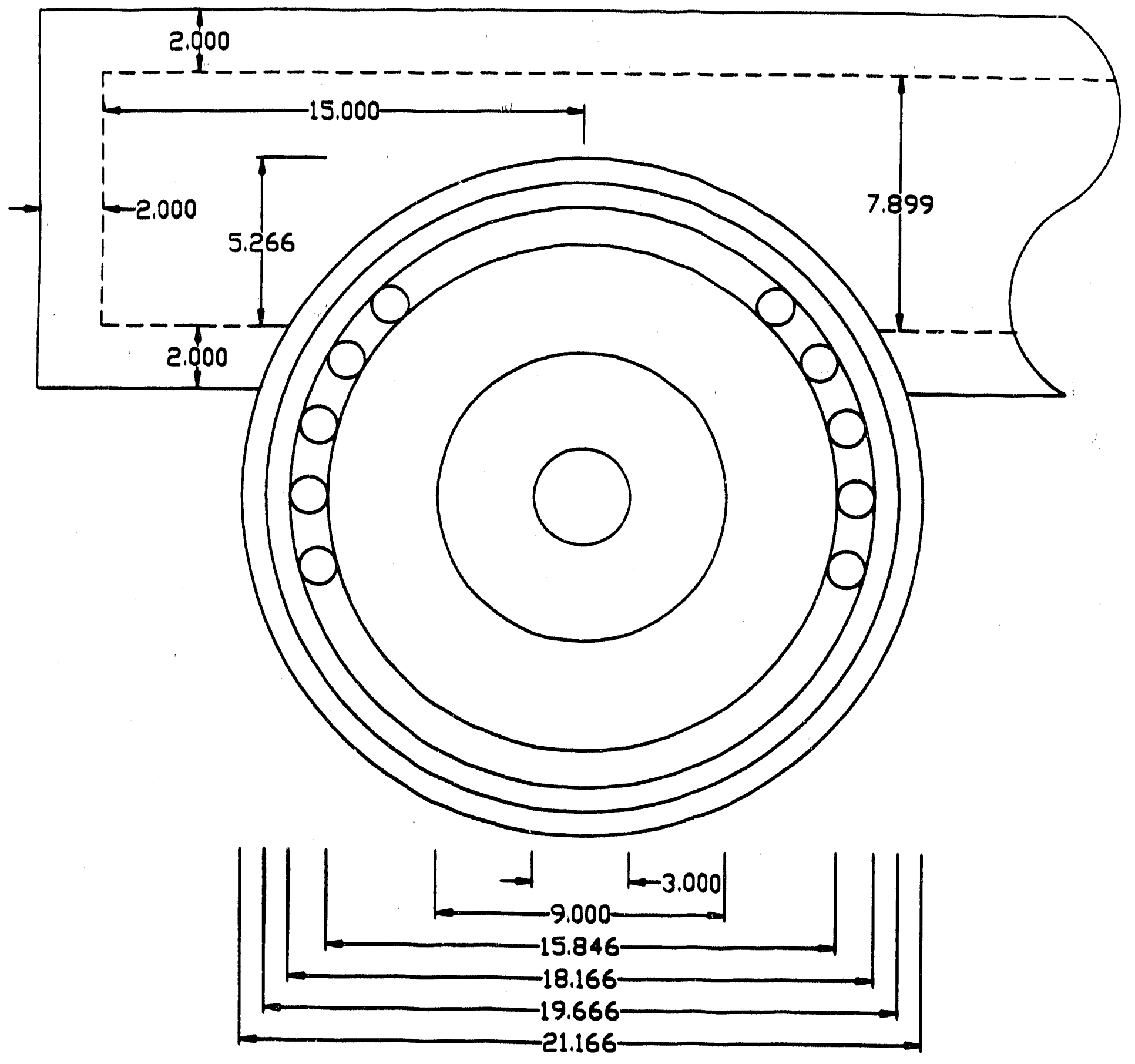

rear view 
$-2.500-$

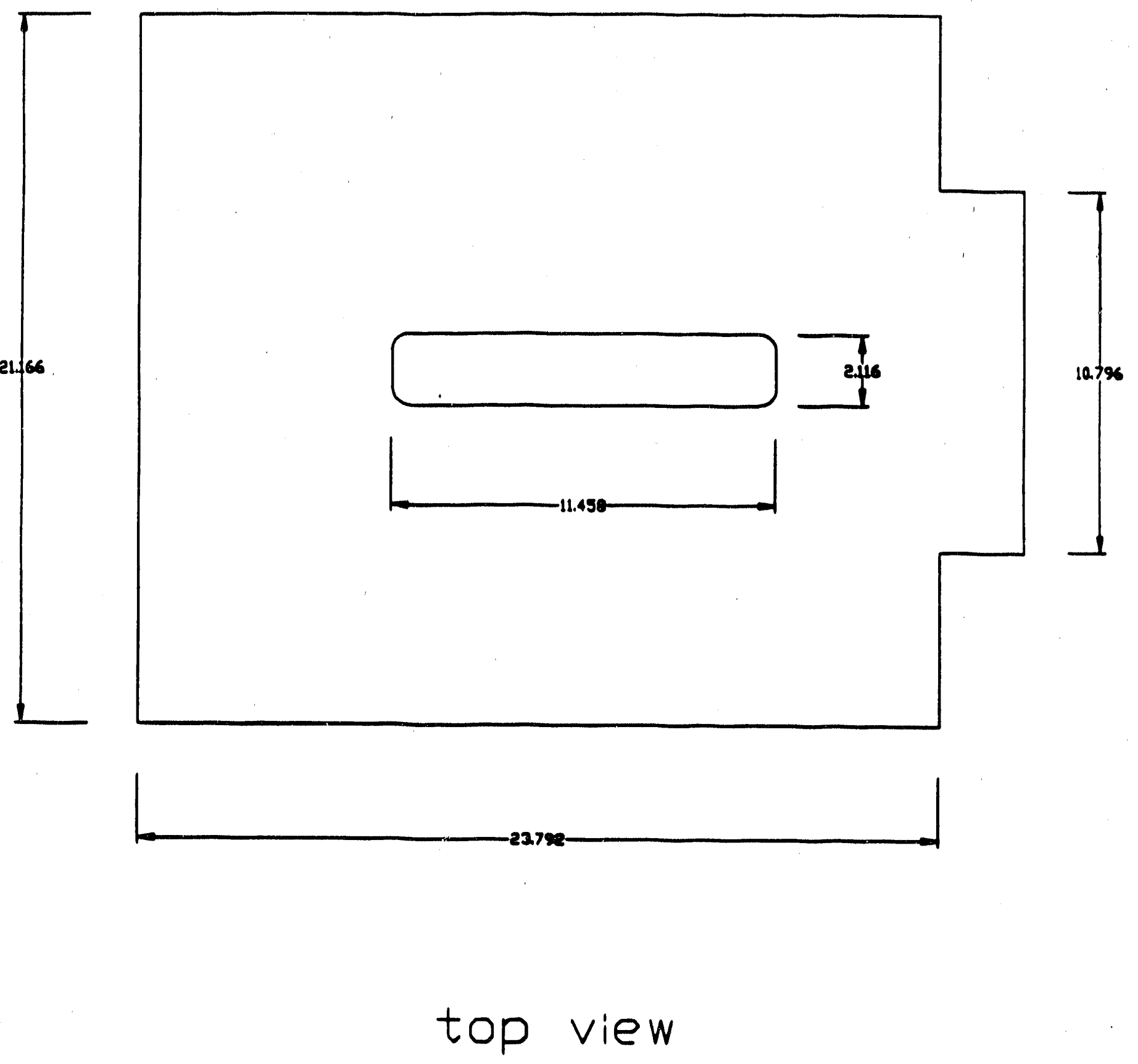




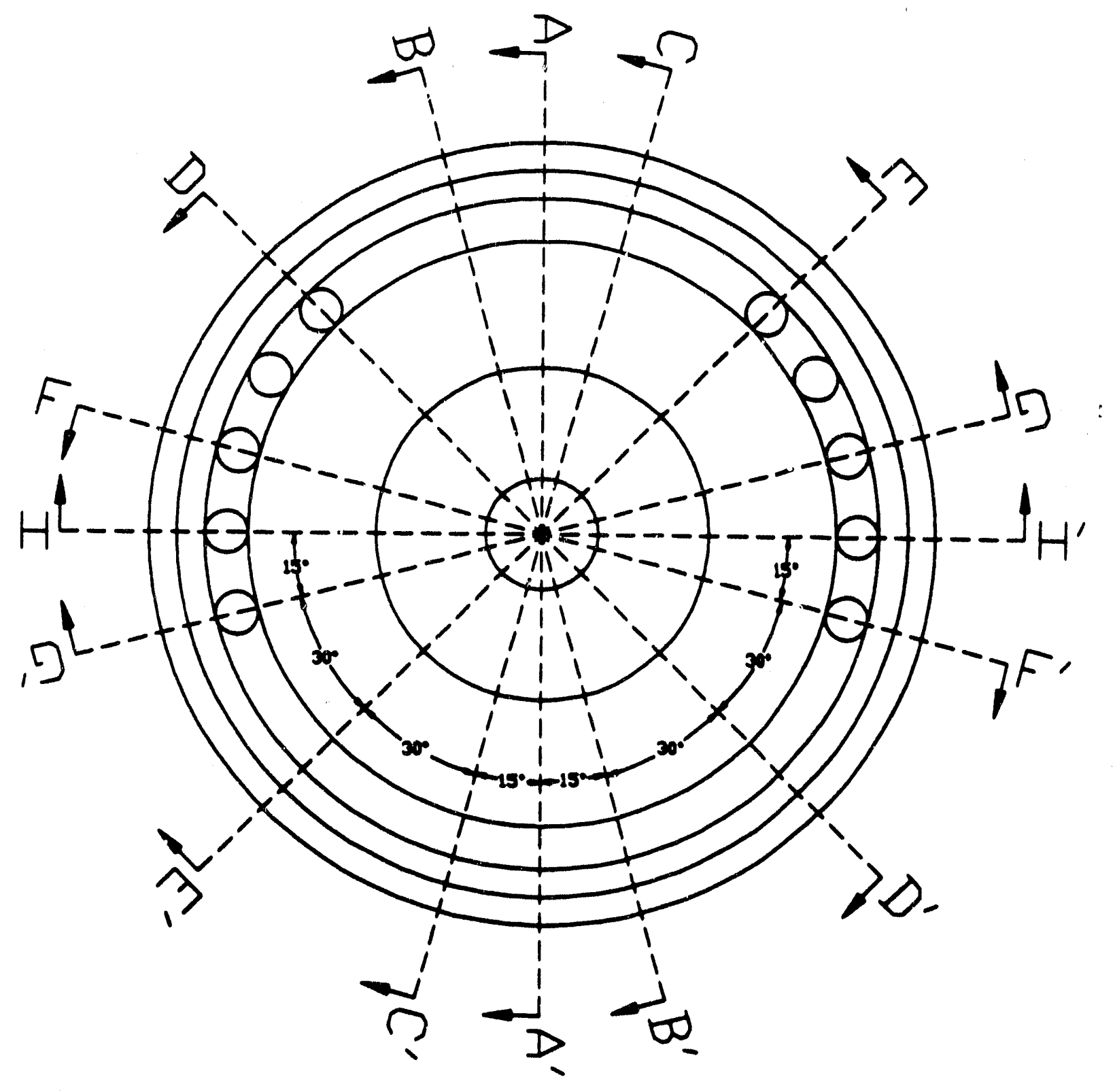

rear view 


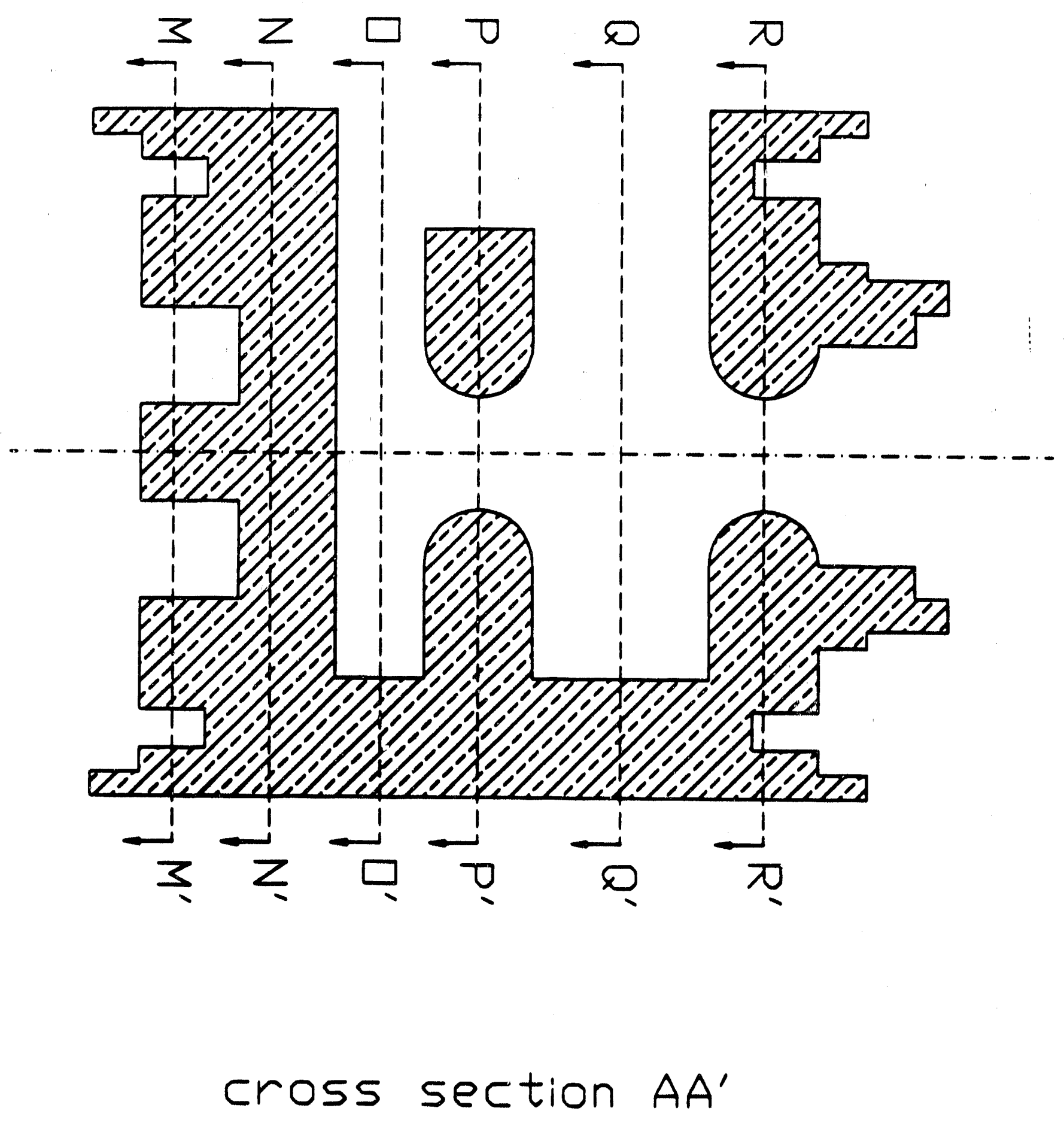




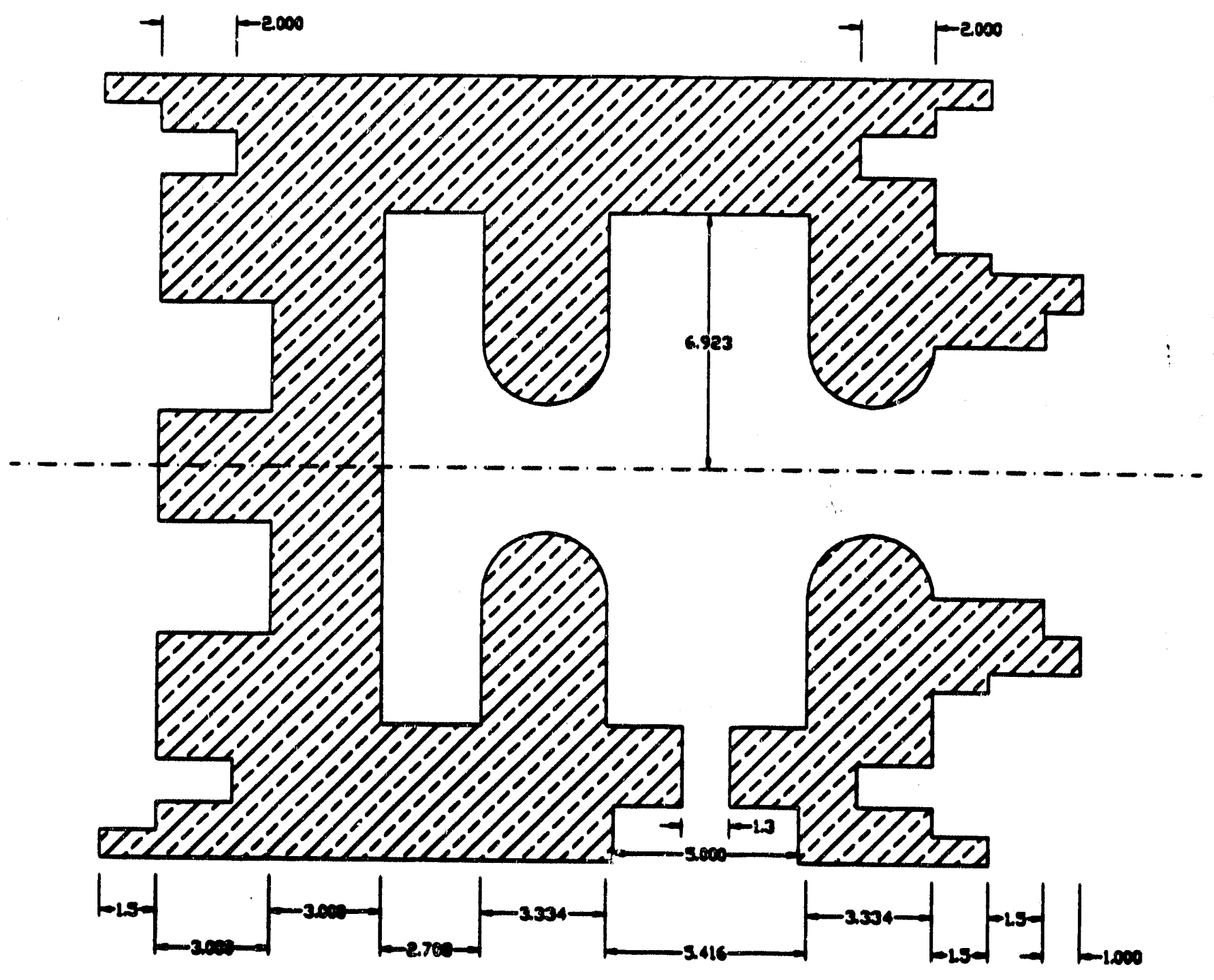

cross section CC' 


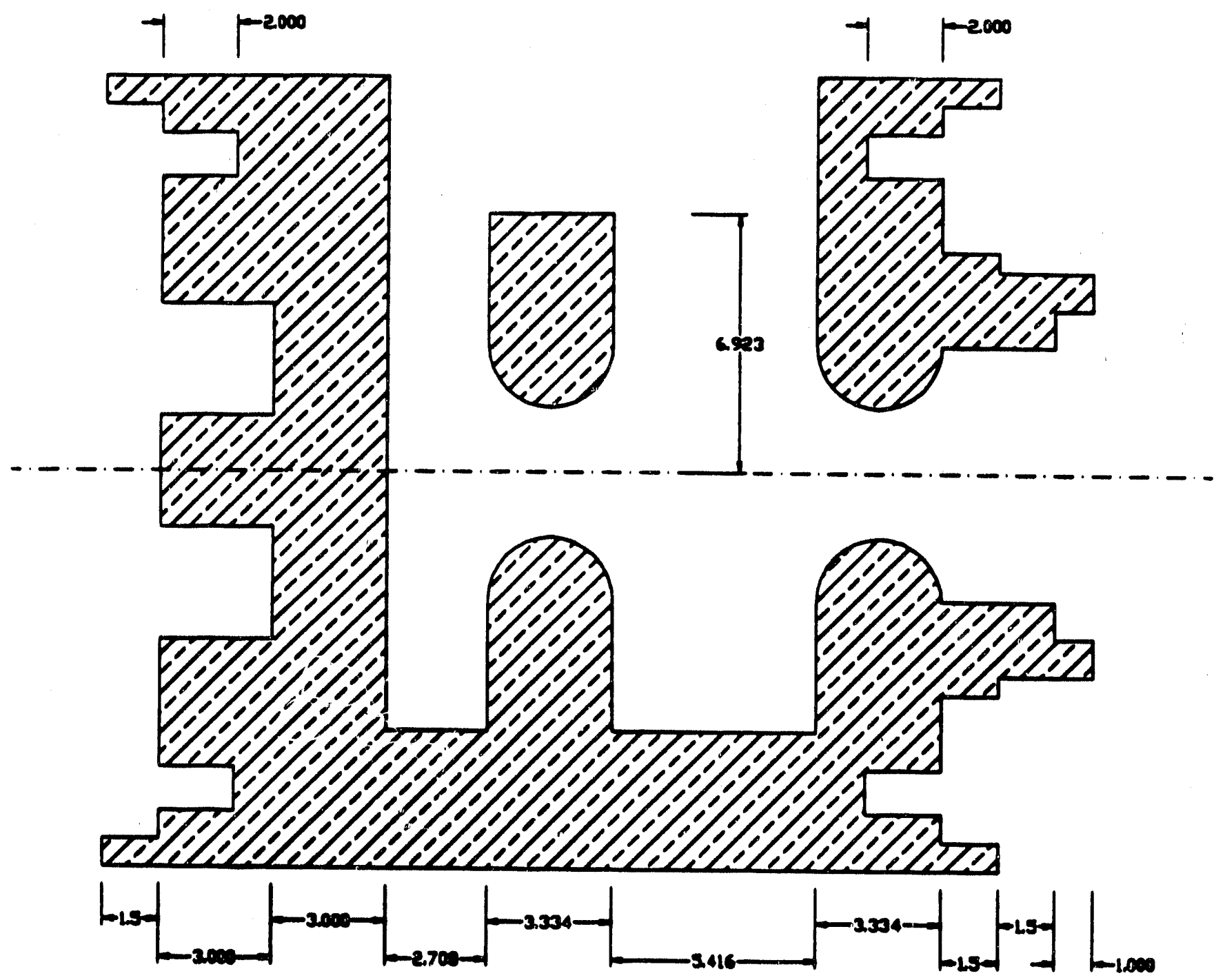

cross section $A A^{\prime}$ 

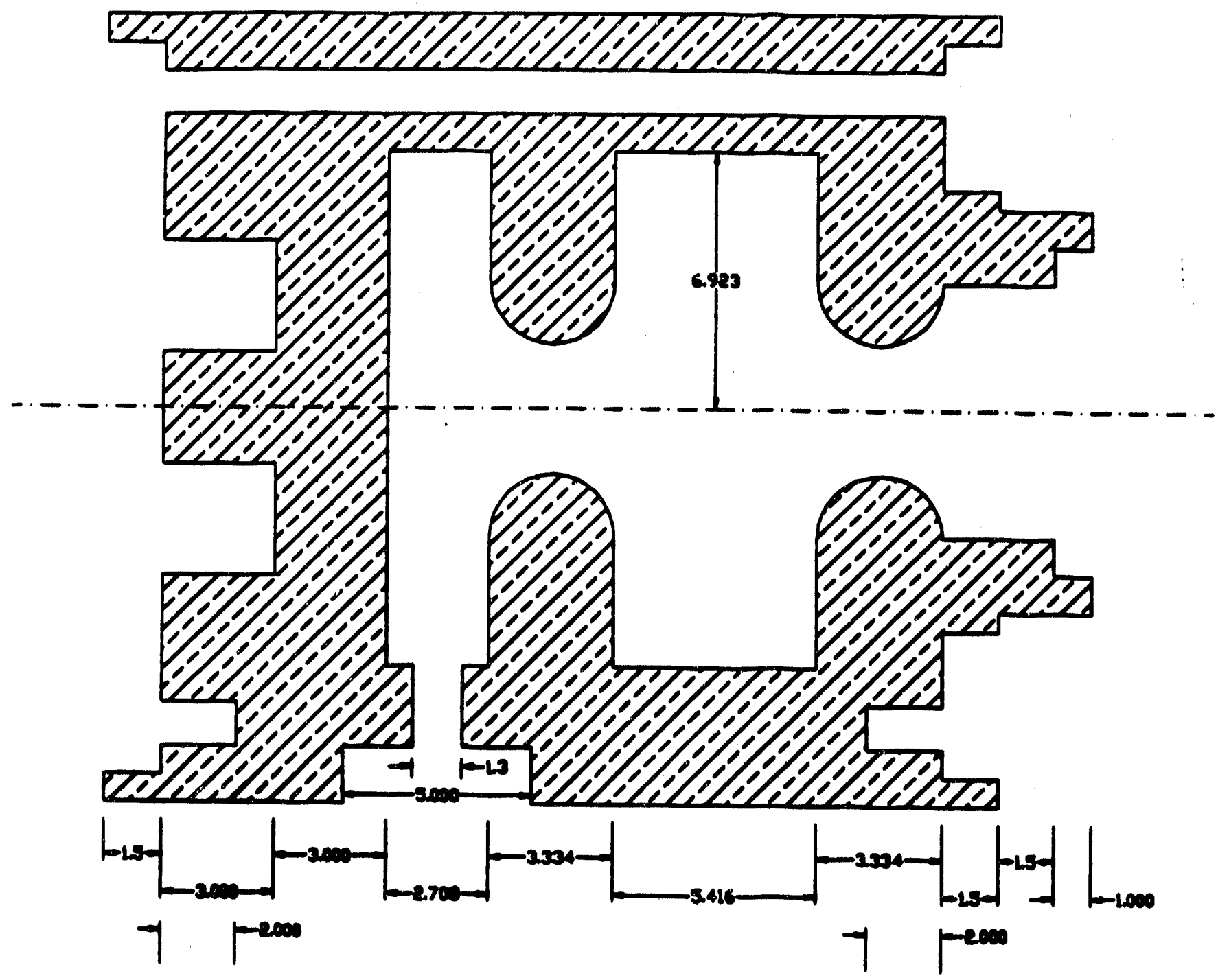

cross section $E E^{\prime}$ 


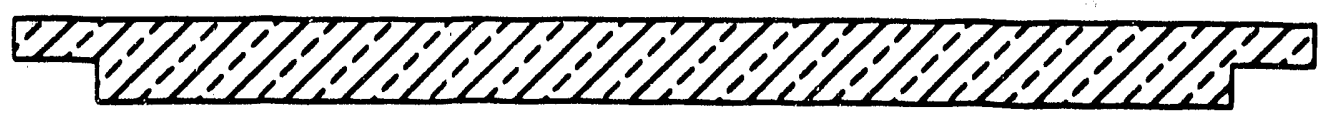
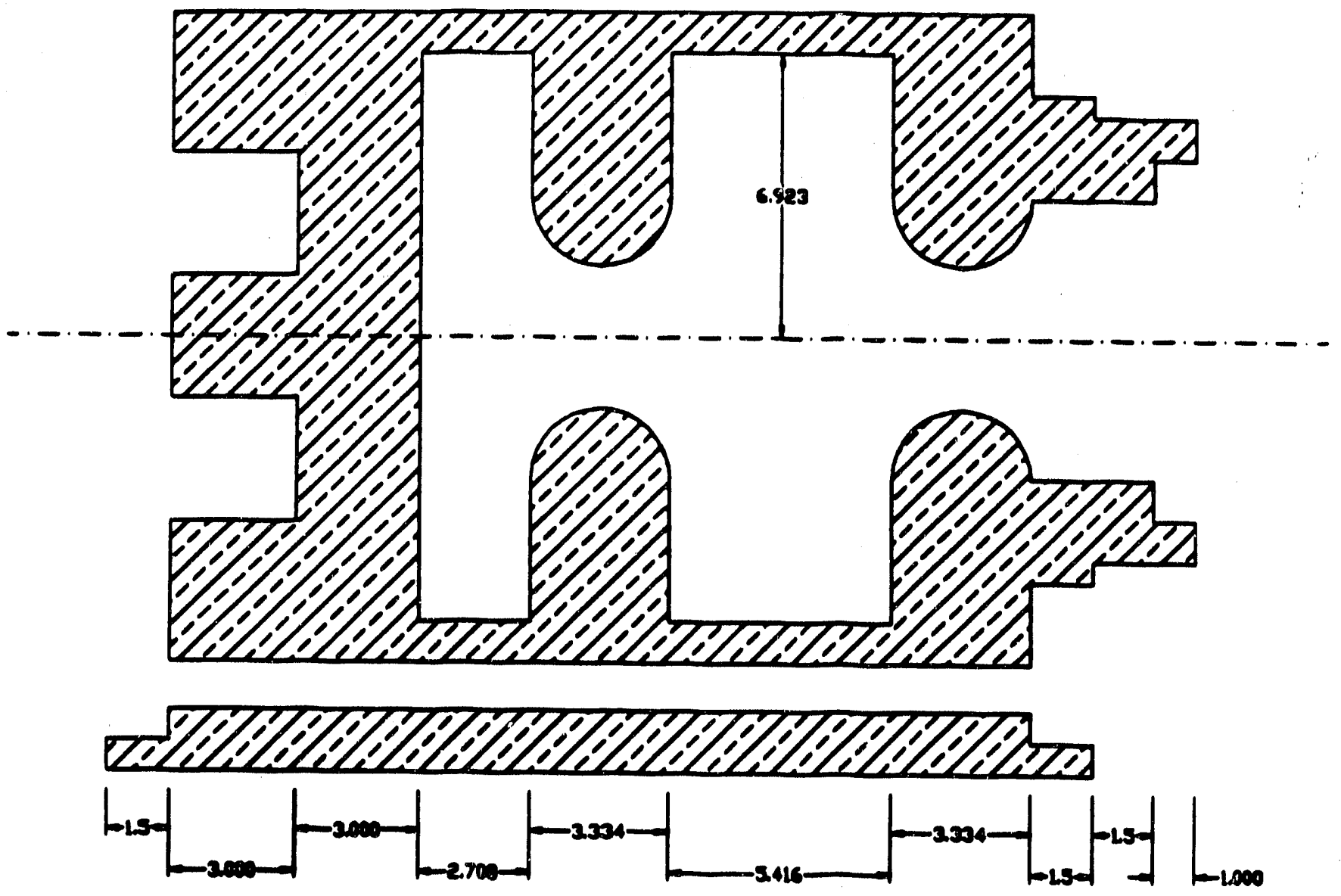

cross section $F F^{\prime}, G G^{\prime}$, and $H H^{\prime}$ 


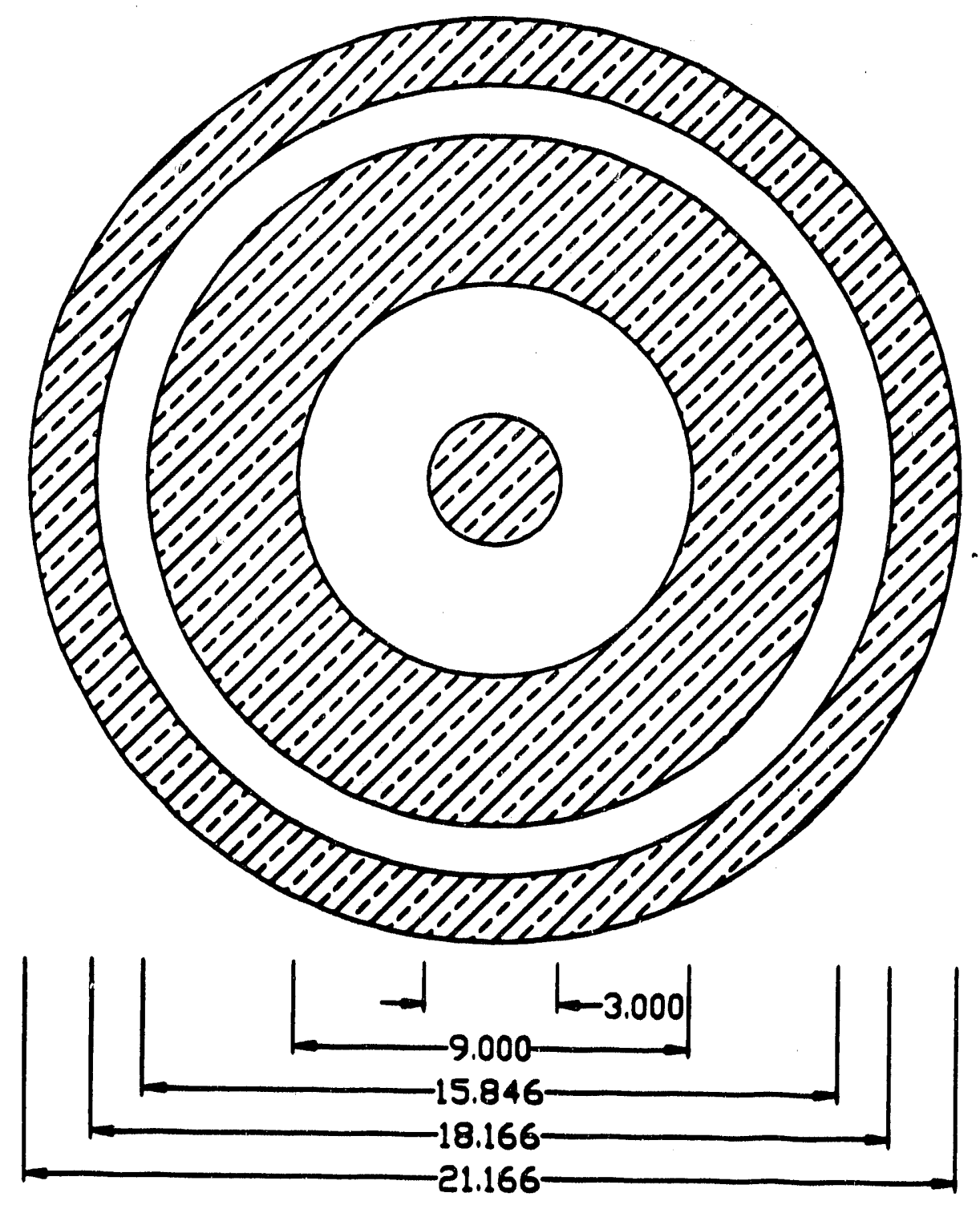

cross section MM' 


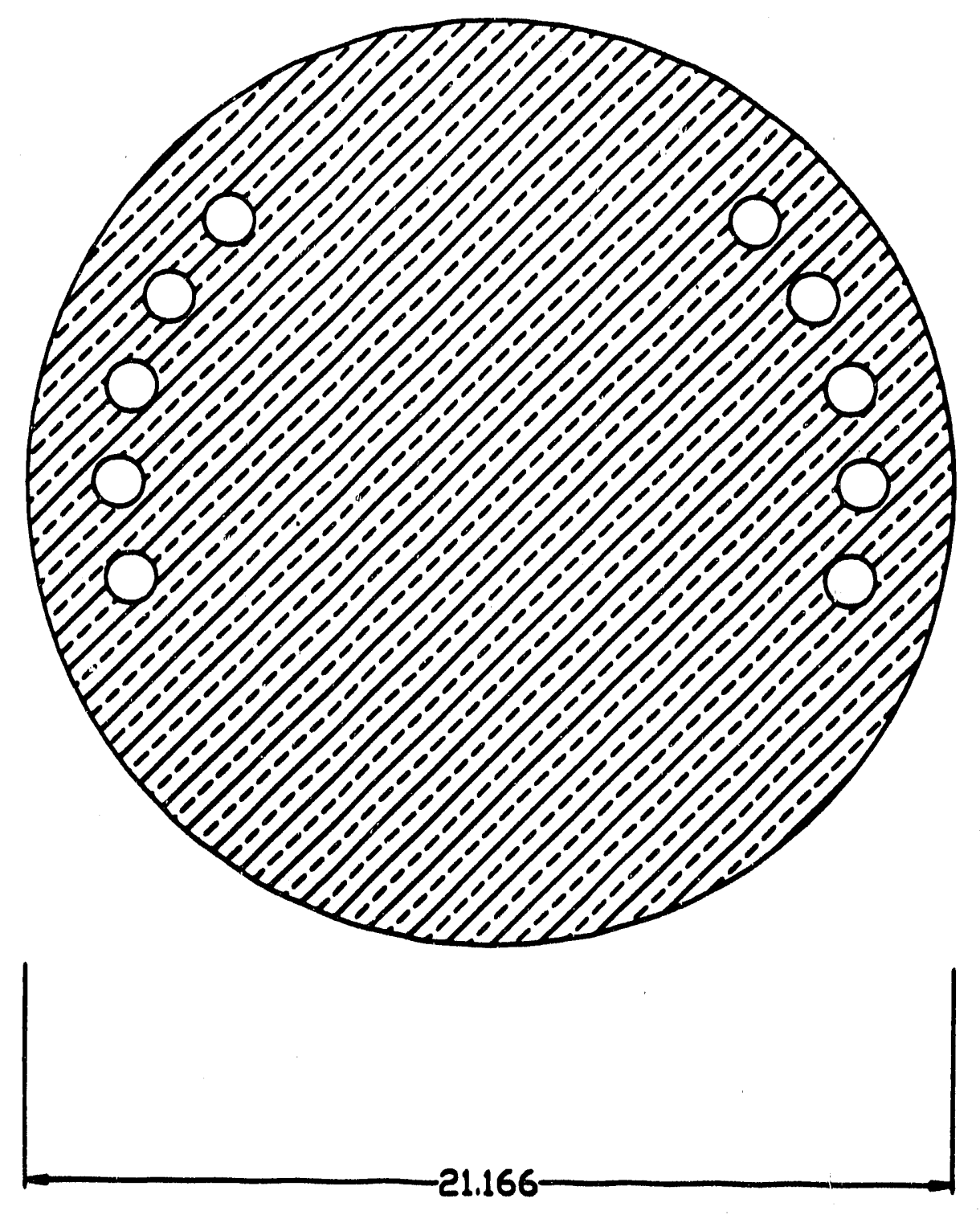

cross section $N^{\prime}$ 


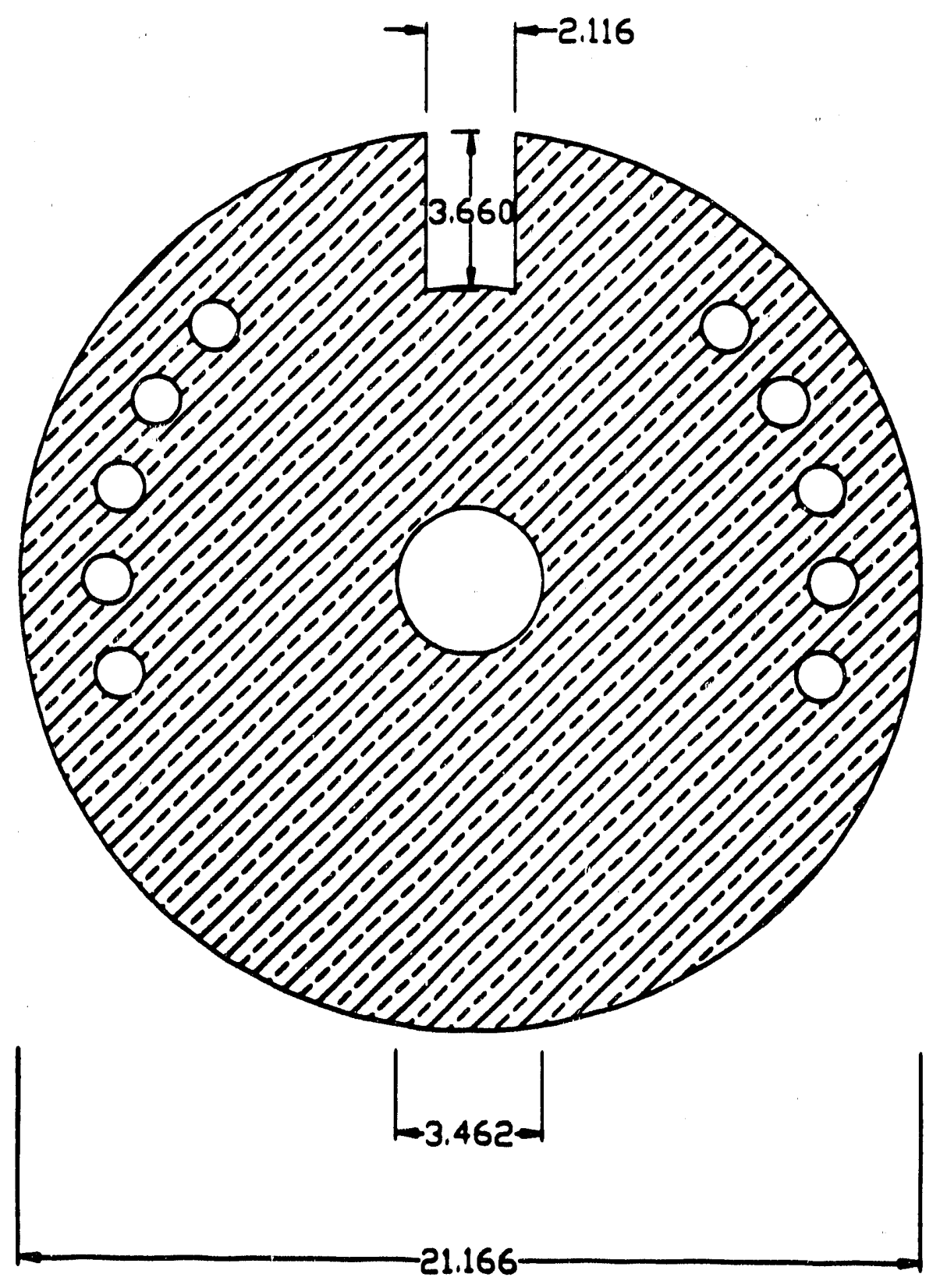

cross section $\mathrm{PP}^{\prime}$ 


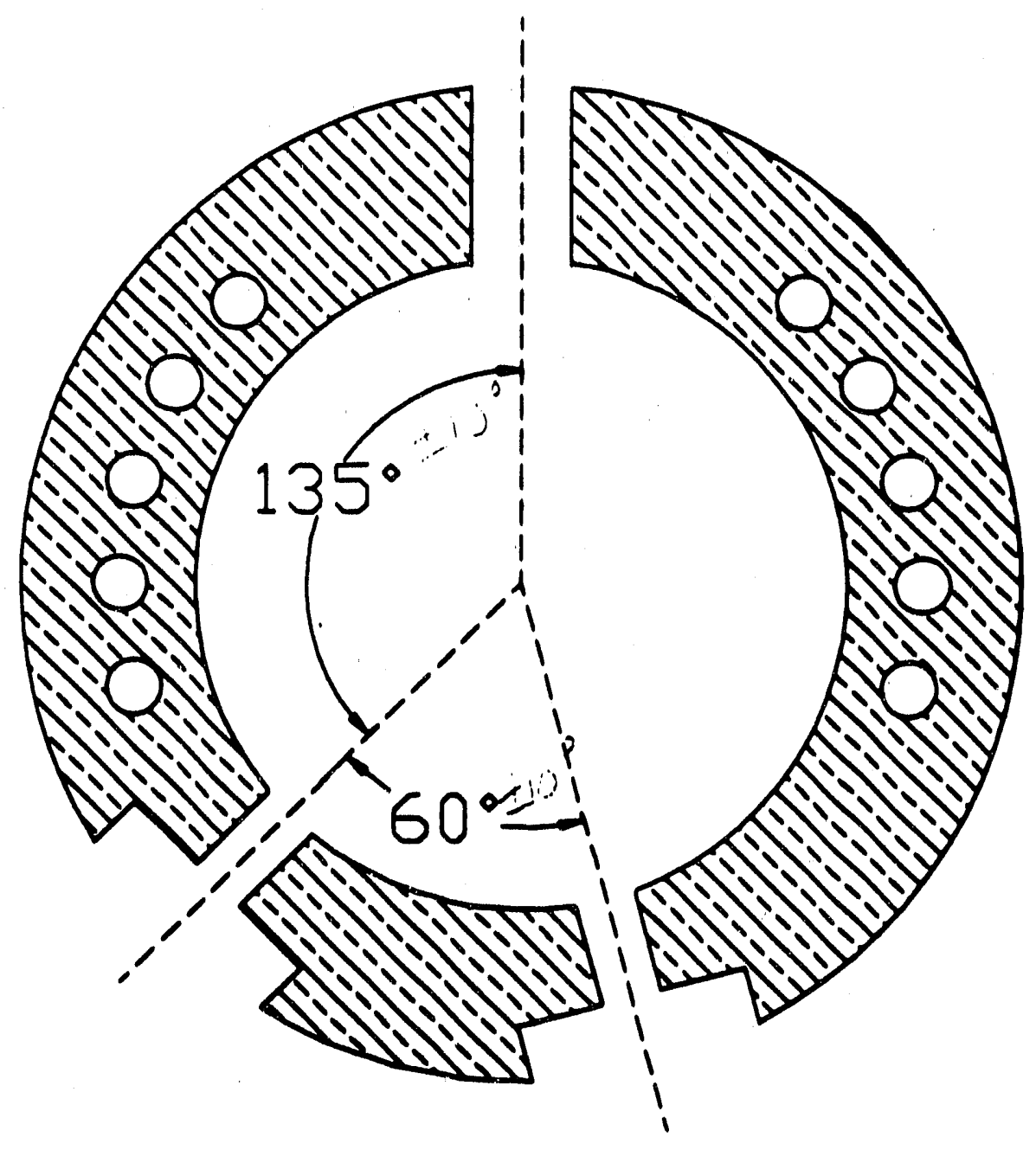

cross section $Q^{\prime}$ (front view) 


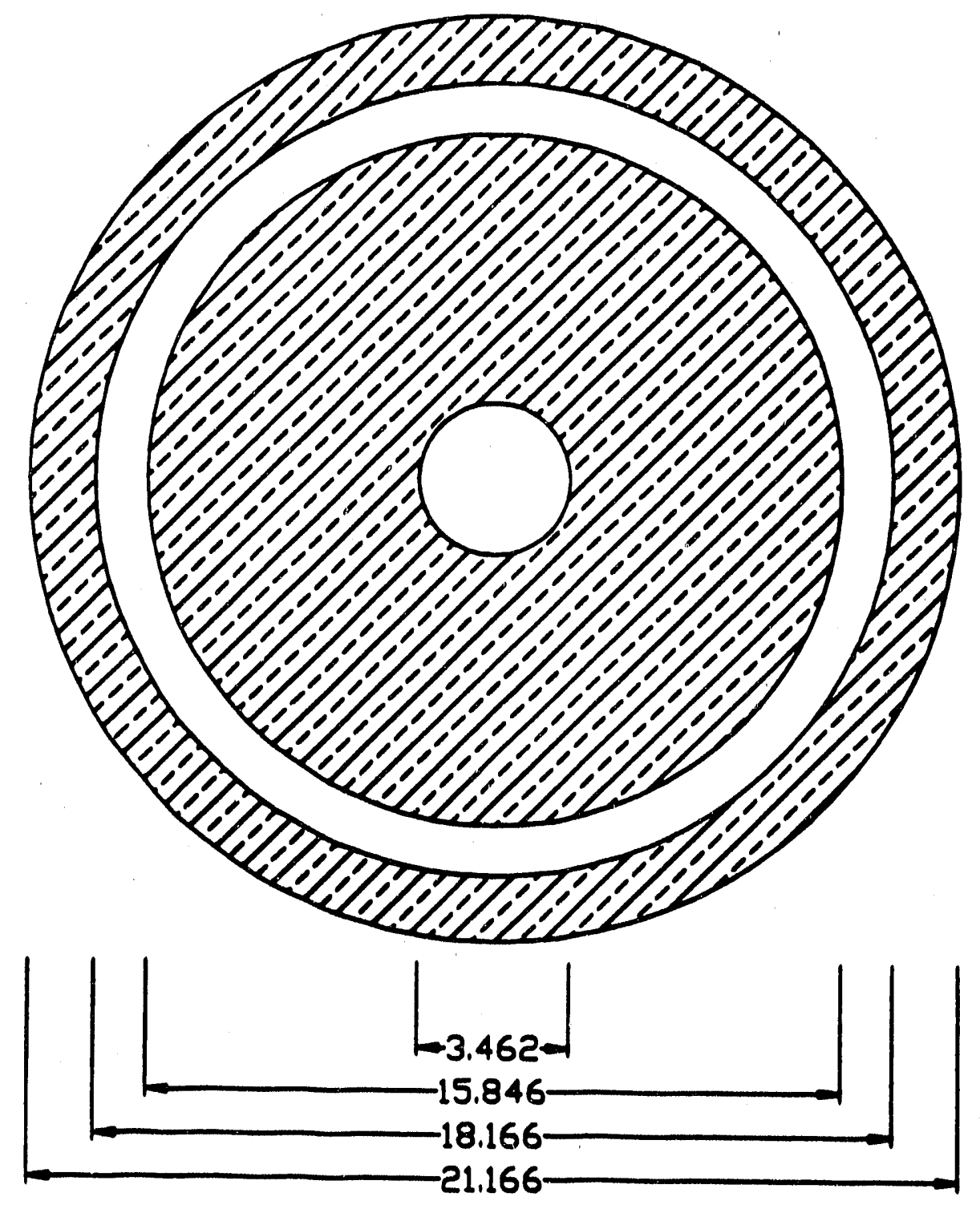

cross section RR' 


\begin{tabular}{|c|c|c|c|c|}
\hline Pump-Line \#1 & 25 deg $C$ & air & & \\
\hline Description & Radius & Length & conductance & Total conduactance \\
\hline \multicolumn{5}{|c|}{ 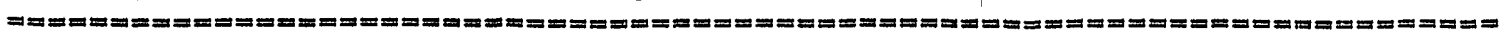 } \\
\hline cavity 1 & 0.6923 & 0.2708 & 15.32 & chamber \\
\hline aperature 1 & 0.3462 & 0.334 & 3.23 & 3.23 \\
\hline cavity 2 & 0.6923 & 0.5416 & 13.59 & 2.61 \\
\hline aperature 2 & 0.3462 & 0.167 & 3.72 & 1.53 \\
\hline exit taper & $?$ & & 8.00 & 1.29 \\
\hline drift tube & 1.75 & 20 & 21.24 & 1.21 \\
\hline diagnostic $T$ & 1.75 & 4 & 60.46 & 1.19 \\
\hline drift tube & 1.75 & 20 & 21.24 & 1.13 \\
\hline diagnostic $\mathrm{T}$ & 1.75 & 4 & 60.46 & 1.11 \\
\hline Ion pump 1 & in line & & 8.00 & 0.97 \\
\hline
\end{tabular}

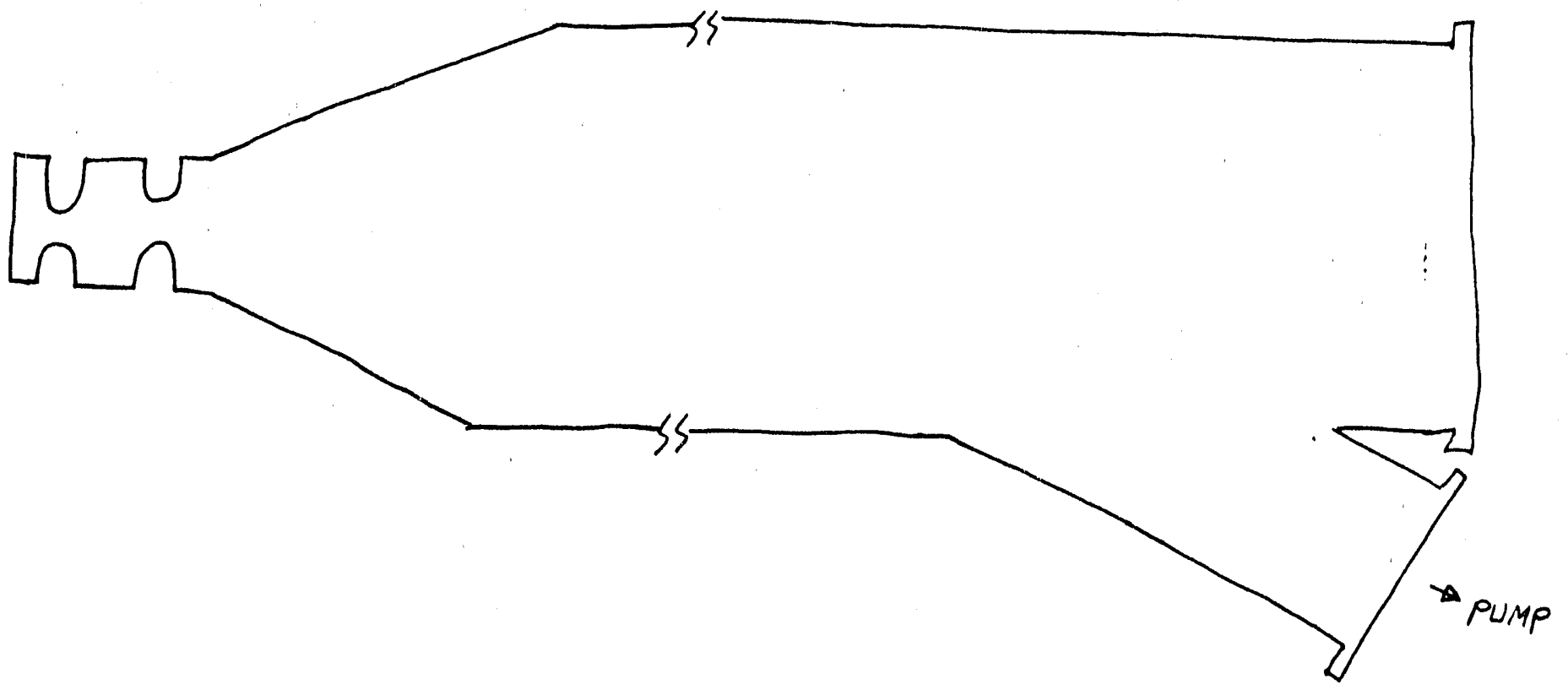




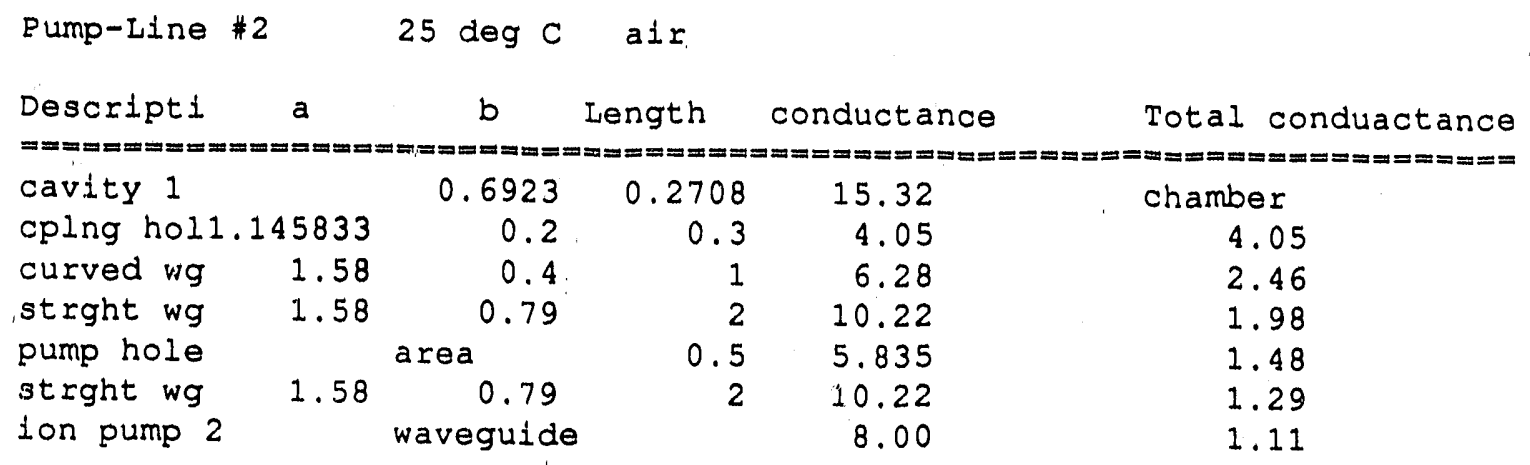

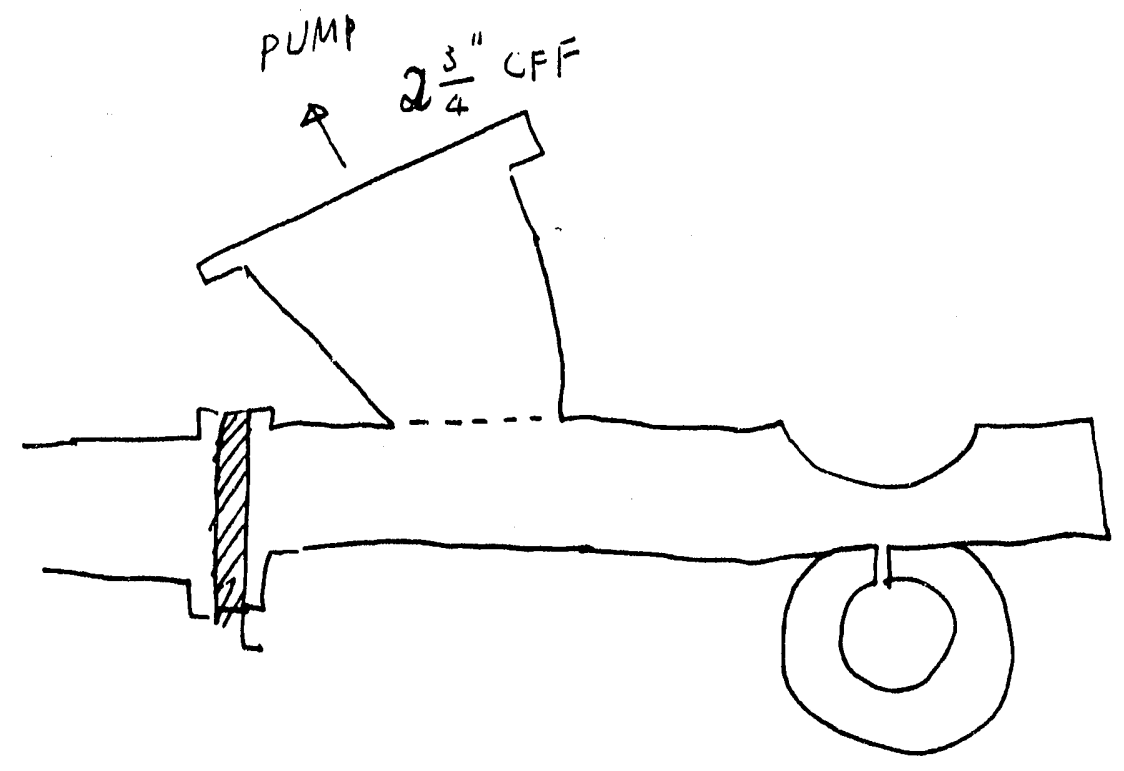


Appendix D. CARM Amplifier Status Report 


\section{CARM Amplifier Status Report}

Operation of the $17 \mathrm{GHz}$ CARM amplifier experiment began during January. RF output power was observed from the first day of operation, but the observed output power was due to amplified spontaneous emisson (ASE) in the amplifier rather than due to amplification of the injected 17 $\mathrm{GHz}$ microwave pulse. During the following several days the frequency of the ASE power was determined to be at approximately $16 \mathrm{GHz}$ with a YIG-tuned filter system. At the present time, we believe that there is insufficient drive power at $17 \mathrm{GHz}$ to overcome the noise power on the beam. The lack of sufficient drive power is attributed to insufficient coupling of the 17 $\mathrm{GHz}$ drive power into the proper mode $\left(\mathrm{TE}_{11}\right)$ for amplification by the CARM interaction. This situation is being rectified at present by two modifications. We are redesigning the input coupler to be a hole-coupled constant phase velocity coupler, and we are arranging to borrow a $135 \mathrm{~kW}$, $17 \mathrm{GHz}$ magnetron driver from LLNL/LBL (A. Sessler) together with a pulse modulator for the magnetron from Dr. Richard Adler of Northstar Research Corp. The factor of 100 increase in drive power should be more than enough to overcome the noise power on the electron beam. The long term solution requires fabrication of the new input coupler which should permit use of the TWT driver as the input source for the CARM amplifier. In the worst case, that the magnetron driver is required even with the new input coupler, we will use the TWT to phase-lock the magnetron. Such a proceedure has been accomplished in our group in the past.

The waveforms resulting from CARM operation to date are shown in Fig. 1. Operation of the CARM in ASE mode at $16 \mathrm{GHz}$ in the $\mathrm{TE}_{11}$ mode is seen in the figure. Other recent experimental work has centered on calibration of the beam diagnostics, and in particular the BDOT and Diamagnetic (DM) loops for determination of the beam current and the beam pitch $\alpha$. Knowledge of these parameters is critical for a full characterization of the CARM device performance. The BDOT loop has been calibrated with a pulsed wire technique, and data has been fit to an equation as shown in Fig. 2, and the resulting computed and actual positions are shown in Fig. 3. This diagnostic is being employed for a determination of beam current and position. 

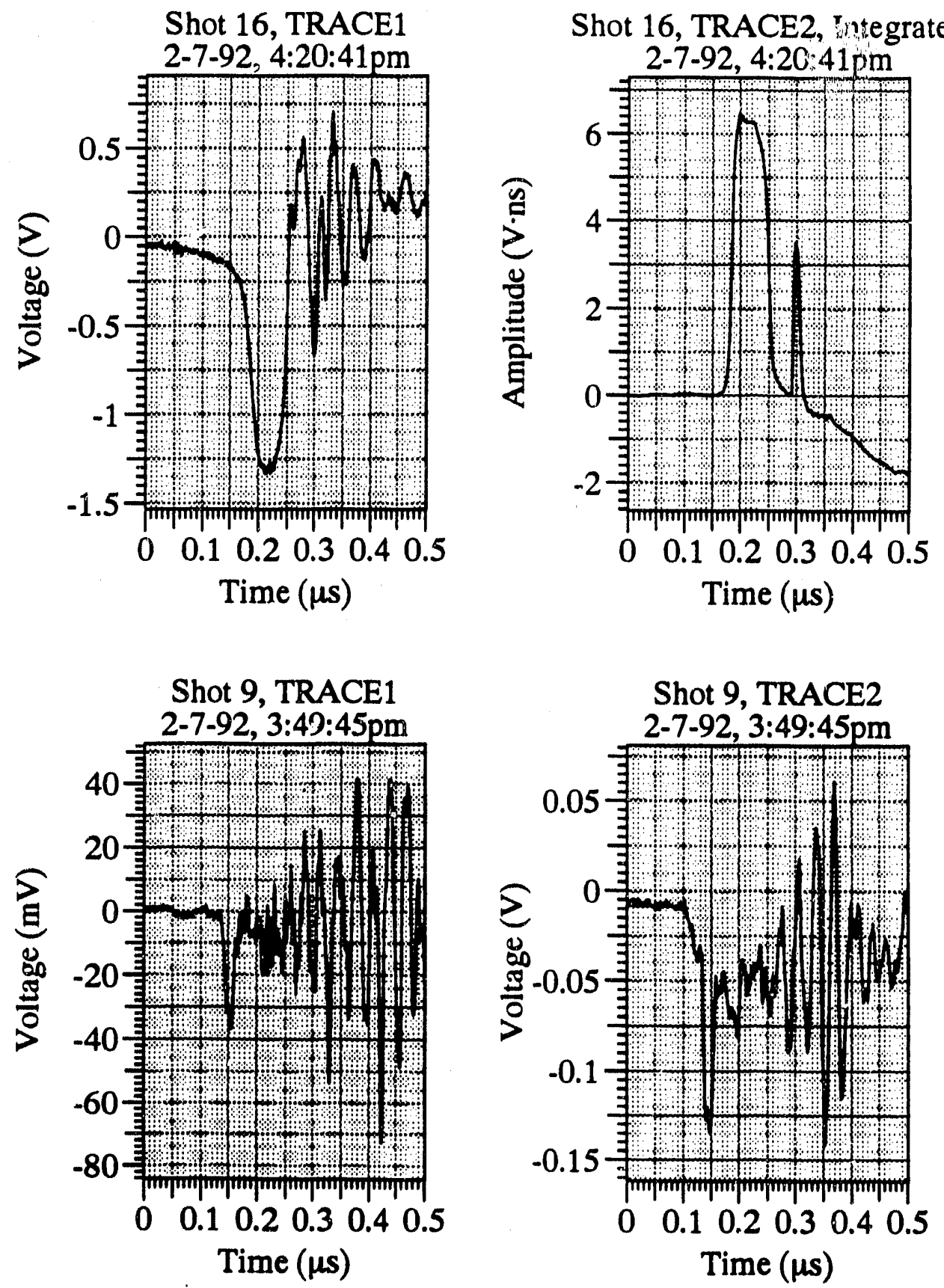

Figure 1: Waveforms from CARM operation. Shot 16, Trace 1 is the voltage waveform, where the calibration is $260 \mathrm{kV} / \mathrm{V}$. Shot 16 , Trace 2 is the integrated signal from the BDOT loop current diagnostic; the calibration is $25 \mathrm{~A} / \mathrm{V}$ ns. Shot 9, Trace 1 is the ASE microwave signal at 16 $\mathrm{GHz}$, and Shot 9, Trace 2 is the microwave signal from a detector and filter tuned to $16 \mathrm{GHz}$. 


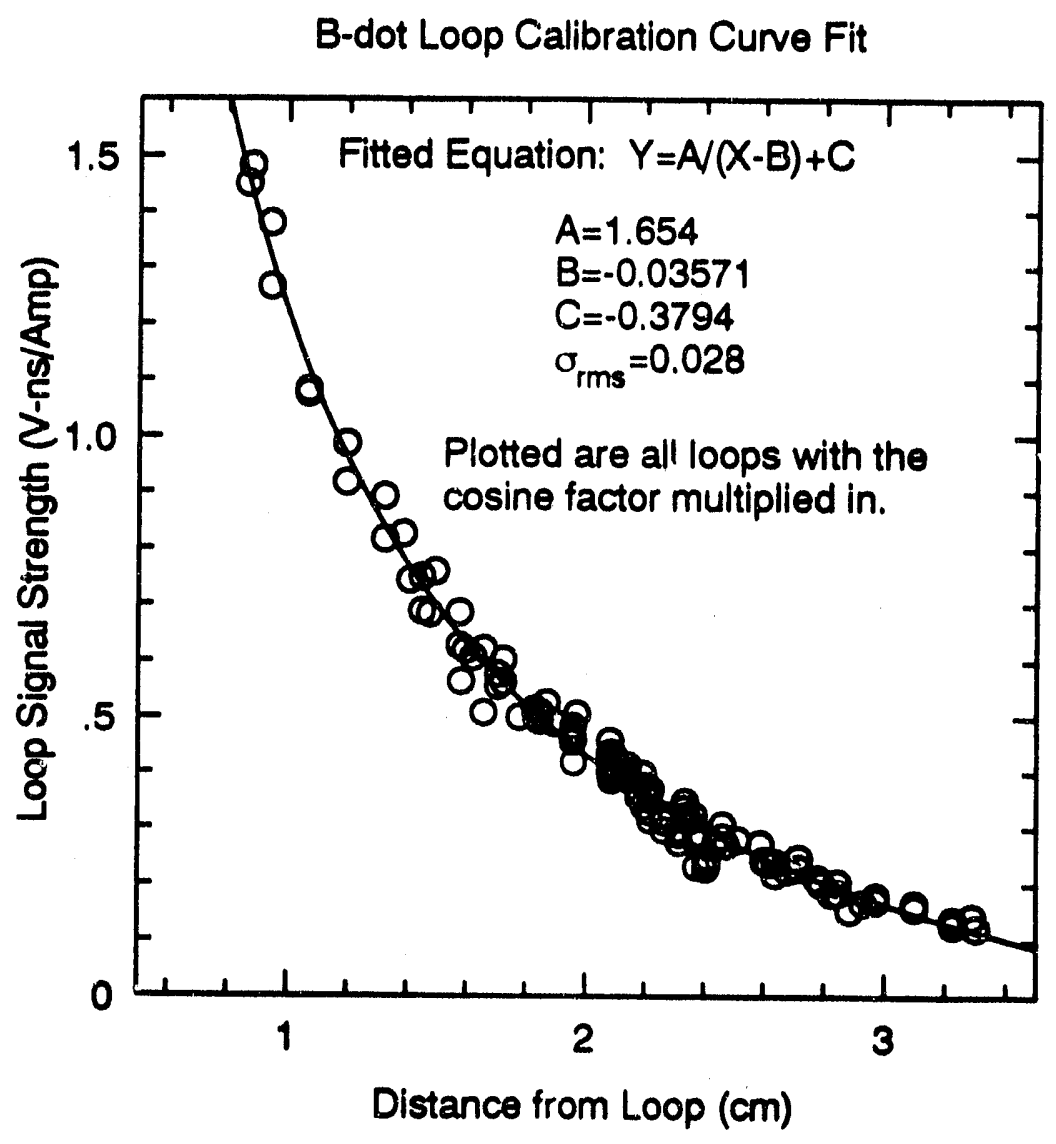

Figure 2: BDOT loop calibration fit of signal strength to pulsed wire position 


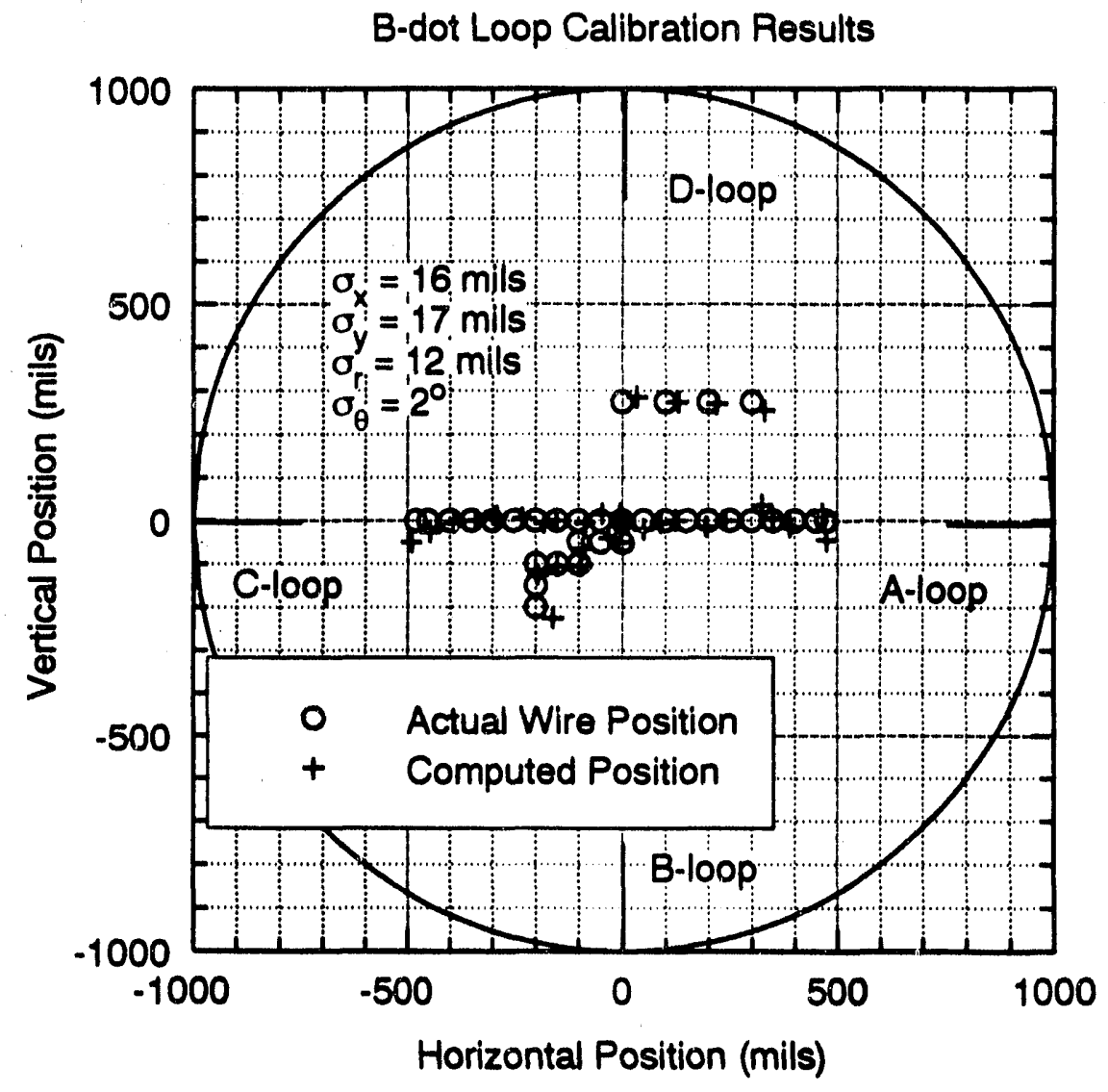

Figure 3: Computed pulsed wire positions and actual pulsed wire positions based upon fit of calibration data. The standard deviations of the calculated position from the actual position are shown. 
Appendix E. 33GHz FEL for Accelerator Applications 
Amplification and Superradiant Emission from a 33.3 GHz Free Electron Laser with a Reversed Axial Guide Magnetic Field

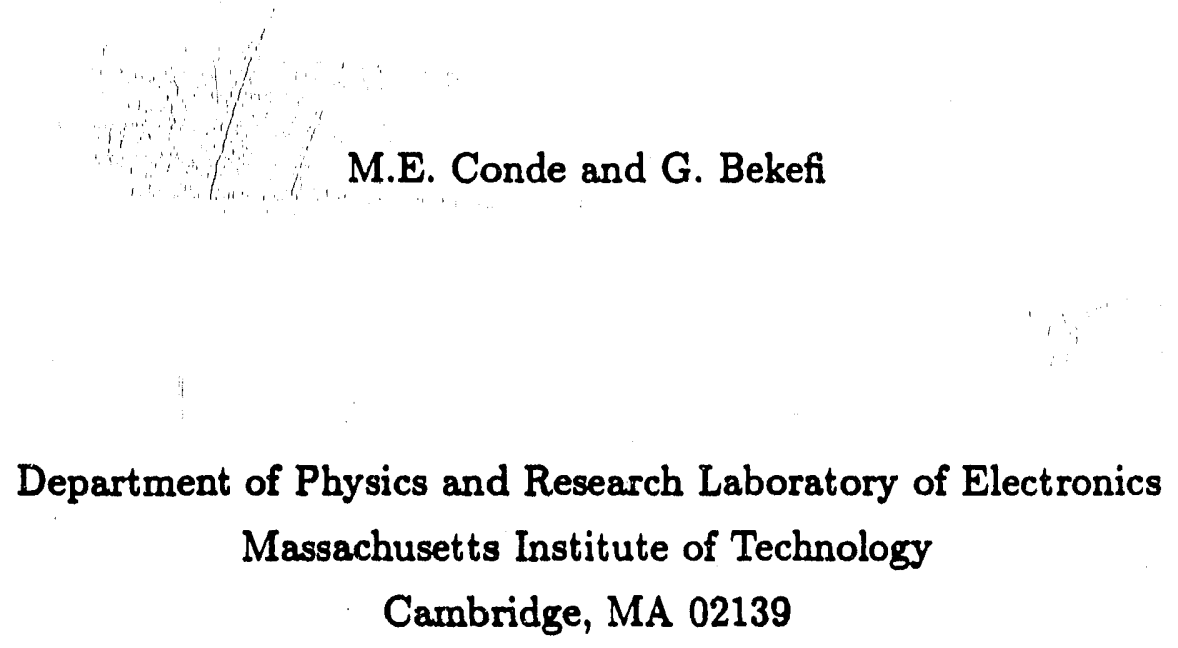

Cambridge, MA 02139

\begin{abstract}
We report on a new regime of free electron laser operation using a helical wiggler field and a reversed axial guide magnetic field. The orientation of the axial field is such as to oppose the rotation of the electrons imparted by the helical field. The $33.3 \mathrm{GHz}$ free electron laser amplifier is driven by a mildly relativistic electron beam $(750 \mathrm{kV}, 300 \mathrm{~A}, 30$ ns) and generates $61 \mathrm{MW}$ of radiation with a $27 \%$ conversion efficiency. The results are compared with those obtained when the axial guide field is in its conventional orientation where considerable loss of power and efficiency is observed.
\end{abstract}




\section{Introduction}

The free electron laser (FEL) operating in a combined axial guide magnetic field and a helical wiggler field has been studied experimentally ${ }^{[1,2]}$ and theoretically ${ }^{[3]}$ over a period of many years, both in linear and nonlinear regimes. In all these studies, the axial magnetic field $B_{z}$ is oriented so that it enhances $[4,5,6]$ the rotation of the beam electrons in the helical wiggler field $\mathbf{B}_{w}$. The increase of the transverse electron velocity $v_{\perp}$, compared to what it would be in the absence of $\mathbf{B}_{\boldsymbol{z}}$, has potential benefits such as an enhanced radiation growth rate and efficiency[3]. Indeed, when the cyclotron wavelength in the axial field $\lambda_{c}=2 \pi v_{z} / \Omega_{z}$, approaches the wiggler periodicity $l_{w}$ the transverse electron excursions can become too large, the electrons strike the drift tube wall and are lost $\left(\Omega_{z}=e \mathrm{~B}_{z} / \mathrm{m}_{0} \gamma\right.$ is the cyclotron frequency in the guide field and $\gamma=\left[1-\left(v_{z} / c\right)^{2}-\left(v_{\perp} / c\right)^{2}\right]^{-1 / 2}$ is the relativistic energy factor). Thus, the "resonance" $\lambda_{c}=l_{w}$ becomes a dividing line for conventional FEL operation: at relatively weak axial fields, $\lambda_{c}>l_{w}$, we have the so called Group I regime, and for stronger fields such that $\lambda_{c}<l_{w}$, the Group II regime.

In this letter we report measurements using a new, hitherto unexplored configuration with a reversed axial magnetic field. The rotation of the electrons in the helical wiggler field $\mathbf{B}_{\omega}$ is now opposed by the presence of the guide field $\mathbf{B}_{z}$ and there is no longer the resonance at $\lambda_{c}=l_{w}$. The transverse electron velocity $v_{\perp}$ is diminished compared to what it would be in the absence of $\mathbf{B}_{3}$, however the latter reduction is partially compensated in our experiments by increasing $\mathbf{B}_{w}$. We will show that a reversal of $\mathbf{B}_{\boldsymbol{z}}$ yields higher radiation intensity and efficiency compared to what we were able to achieve with the conventional orientation of the axial magnetic field.

The three regimes of FEL operation (Group I, Group II, and Reversed Field) are displayed in Fig. 1 based on a particle trajectory calculation of $v_{z} / c$ which neglects space charge and radiation, and assumes that the electrons are undergoing ideal helical orbits in the combined $B_{z}$ and $B_{w}$ fields. The solid points illustrate the three parameter regimes of $B_{z}$ where maximum radiation has been observed in our experiments.

\section{Experiment}

A schematic of the FEL amplifier is shown in Fig. 2. A mildly relativistic electron beam $(750 \pm 50 \mathrm{keV}$ ) is generated by a Marx capacitor bank (Physics International Pulserad 
110 A). The electrons are emitted from a hemispherical graphite cathode by an explosive field emission process. The graphite anode acts as an emittance selector, allowing only a small fraction of the current to propagate through its $2.54 \mathrm{~mm}$ radius and $62 \mathrm{~mm}$ long aperture. The electron beam current downstream from the emittance selector is illustrated in Fig. 3, showing saturation at high $B_{z}$ where all available electrons from the gun have made it through the anode hole. From such measurements we are able to estimate ${ }^{[7]}$ the normallized RMS beam emittance $\epsilon_{n}<4.4 \times 10^{-2} \mathrm{~cm}$-rad and the corresponding RMS axial energy spread $\Delta \gamma_{z} / \gamma_{z}<1.5 \times 10^{-2}$. We observe from Fig. 3b that when the wiggler is turned on, an expected very pronounced current loss occurs near resonance $\lambda_{c}=l_{w}$ with the conventional orientation of $B_{z}$, but no significant current loss (also as expected), with the magnetic field reversed (Fig. 3c).

The 50 period bifilar helical wiggler produced by current carrying helical wires has a period of $3.18 \mathrm{~cm}$ and provides a magnetic field whose magnitude on axis is adjustable up to $1.8 \mathrm{kG}$. The wiggler field is slowly up-tapered over the initial six periods, providing an adiabatic input for the electron beam. The system, including the gun, is immersed in a uniform axial magnetic field generated by a solenoid. The intensity of this field can be varied up to a maximum of $11.6 \mathrm{kG}$.

The 2 meter long stainless steel drift tube has an internal radius of $0.51 \mathrm{~cm}$ and acts as a cylindrical waveguide whose fundamental $T E_{11}$ mode has a cutoff frequency of 17.2 GHz. The system is designed to operate in this lowest waveguide mode.

A high power magnetron operating at $33.39 \mathrm{GHz}$ is the input power source for the FEL amplifier. The wave launcher consists of a short section of circular waveguide of radius 0.31 $\mathrm{cm}$ into which $17 \mathrm{~kW}$ are coupled from a standard Ka-band rectangular waveguide. This section of circular waveguide supports only the fundamental $T E_{11}$ mode for the operating frequency. Its radius is then adiabatically up-tapered to the radius of the drift tube. A linearly polarized wave is thereby injected into the interaction region. Half of the incident power, with the correct rotation of the electric field vector, participates in the FEL interaction.

The outpat power from the FEL is transmitted by means of a conical horn into a reflection free "anechoic chamber". A small fraction of the radiation is then collected by a 
receiving horn, passes through precision calibrated attenuators, and a $1.7 \mathrm{GHz}$ wide band pass filter. The power level is finally determined from the response of a calibrated crystal detector. The entire system is calibrated absolutely by a substitution method, i.e., by turning off the electron beam and measuring the received power in terms of the known input power from the magnetron.

Angular scans of the radiation pattern of the transmitting horn (Fig. 4) carried out within the anechoic chamber, together with a set of high pass waveguide filters are used to confirm that the FEL indeed operates in the $T E_{11}$ waveguide mode. The frequency spectrum of the output power has also been determined by a heterodyne technique (see Fig. 2). A crystal rectifier is used as a mixer for the $33 \mathrm{GHz}$ FEL radiation and for radiation from a variable frequency $23 \mathrm{GHz}$ local oscillator. The beat wave is amplified by a TWT amplifier and sent into a filter bank, composed of 13 channels adjacent in frequency, each $80 \mathrm{MHz}$ wide. Thus, on a single shot basis, the radiation frequency is determined to be 33.39 $\mathrm{GHz}$ with a full width at half maximum of less than $160 \mathrm{MHz}$ (Fig. 5).

A parameter scan of the output power has been carried out in order to discover the optimum operating conditions for our three regimes, Group I, Group II and Reversed Field. Figure $6 a$ illustrates the output power as a function of $B_{w}$ at constant $B_{z}$, and Figs. $6 b$ and $6 c$ show how the power varies with $B_{z}$ at constant $B_{w}$. It is seen that the maximum output power obtained in Group I and Group II regimes is approximately the same, 5 MW; however, the efficiency is much higher for the Group I regime since here the beam current is smaller. The output power for the reversed field case is higher by an order of magnitude and reaches a level of $61 \mathrm{MW}$.

The spatial growth rate of the electromagnetic wave is determined from the measurement of the output power as a function of the length of the interaction region. This length is varied by changing the distance that the electron beam is allowed to propagate in the drift tube. Application of a strong magnetic field is sufficient to deflect the electrons into the waveguide wall and thereby terminate the interaction at that point. Figure 7 shows the result of this measurement for the three different regimes. The Group I regime has the highest small signal growth rate $(44 \mathrm{~dB} / \mathrm{m})$ but the power level reaches saturation at an efficiency of $9 \%$. Operation in Group II shows the lowest growth rate $(38 \mathrm{~dB} / \mathrm{m})$ and the 
lowest efficiency (2\%). The Reversed Field operation has an intermediate growth rate (41 $\mathrm{dB} / \mathrm{m}$ ) but by far the highest efficiency (27\%), and exhibits, no power saturation. These values of the growth rates apply only in the limited range indicated by the straight lines shown in Fig. 7. They represent best fits to exponential power. Figure 7 also shows that in the Group I regime the spatial startup is much shorter than in the other two regimes. The reason for this difference is not yet understood.

Radiation studies in the superradiant regime, i.e. in the absence of the driver magnetron, have also been carried out. Figure 8a shows a scan of output power as function of the wiggler strength $B_{w}$ in the Group I regime. We see that the maximum output is of the order of $1 \mathrm{MW}$. The spectrum characteristics using our spectrum analyzer are shown in Fig. 8b. As expected, the frequency spectrum is much broader and less repetitive when the FEL is not driven by the magnetron.

Superradiant measurements in Group II and Reversed Field regimes were not carried out because the observed power levels were too low $(<20 \mathrm{~kW})$.

\section{Discussion}

In conclusion, we have found that our free electron laser shows highest efficiency and highest power output when operated with reversed axial guide magnetic field. Table I summarizes our findings. We believe that the $27 \%$ efficiency of our system exceeds the efficiency of previous FELs with untapered wigglers. If the $\sim 1 \mathrm{~dB} / \mathrm{m}$ attenuation due to the stainless steel waveguide were subtracted from the measurements, the power and efficiency could be substantially higher. Moreover, Fig. 7c shows that our wiggler is too short to reach saturation, and much higher efficiency may well be possible with longer and/or tapered magnetic fields.

We note that our FEL falls into the so called Raman parameter regime where RF space charge effects must be allowed for, both in order to account for the radiation frequency and the power output. A detailed explanation of the Reversed Field FEL experiments requires modification of the ideal orbit theory on which Fig. 1 is based. This fact is illustrated in Fig. 9 in which the solid points ..present numerical simulations ${ }^{\left[{ }^{[8}\right.}$ of the electron trajectories. The departure from ideal orbit theory is particularly noticeable at a magnetic field $B_{z} \simeq-7.5 k G$ where a dip in $v_{z} / c$ is observed and corresponds to a 
novel "anti-resonance" condition $l_{w} \simeq-\lambda_{c}$ recently described in references [9] and [10]. This is due to the finite thickness of the electron beam in which the off-axis electrons undergo betatron-like oscillations. The presence of the anti-resonance manifests itself as a hundred-fold decrease in the output power seen as a sharp dip in Fig. 6c. This is caused by a decrease in $v_{z}$, detuning of the FEL and a subsequent intensity degradation.

We note that in the superradiant regime the output powers in Group II and Reversed Field regimes are two orders of magnitude lower than in Group I regime. This must be due in part to the fact that in the Group I regime the radiation grows out of a much more intense pool of background noise.

This study was supported by DOE, AFOSR and CNPq (Brazil). We thank I. Mastovsky, C.J. Taylor and T. Mizuno for the assistance in the experimental work. 
Table I. Summary of experimental results.

\begin{tabular}{lccc}
\hline PARAMETER & GROUP I & GROUP II & REVERSED FIELD \\
Driver frequency (GHz) & 33.39 & 33.39 & 33.39 \\
Beam energy (keV) & 750 & 750 & 750 \\
Beam current (A) & 90 & 300 & 300 \\
Guide field (kG) & 4.06 & 10.9 & -10.9 \\
Wiggler field (kG) & 0.63 & 0.63 & 1.47 \\
Growth rate (dB/m) & 44 & 38 & 41 \\
Output power (MW) & 5.8 & 4.2 & 61 \\
Efficiency (\%) & 9 & 2 & 27 \\
\hline
\end{tabular}




\section{REFERENCES}

[1] S.H. Gold, D.L. Hardesty, A.K. Kinkead, L.R. Barnett and V.L. Granatstein,"HighGain 35-GHz Free-Electron Laser-Amplifier Experiment," Phys. Rev. Lett., vol. 52, no. 14, pp. 1218-1221, 1984.

[2] J. Fajans, J.S. Wurtele, G. Bekefi, D.S. Knowles and K. Xu, "Nonlinear Power Saturation and Phase (Wave Refractive Index) in a Collective Free-Electron Laser Amplifier," Phys. Rev. Lett., vol. 57, no. 5, pp. 579-582, 1986; also, J. Fajans, G. Bekef, Y.Z. Yin and B. Lax, "Microwave studies of a tunable free-electron laser in combined axial and wiggler magnetic fields," Phys. Fluids, vol. 28, no. 6, pp. 1995-2006, 1985.

[3] A.K. Ganguly and H.P. Freund, "High-Efficiency Operation of Free-Electron-Laser Amplifiers," IEEE Trans. Plasma Sci., vol. 16, no. 2, pp. 167-171, 1988.

[4] L. Friedland, "Electron beam dyna:mics in combined guide and pump magnetic fields for free electron laser applications," Phys. Fluids, vol. 23, no. 12, pp. 2376-2382, 1980,

[5] P. Diament, "Electron orbits and stability in realizable and unrealizable wigglers of free-electron lasers," Phys. Rev. A, vol. 23, no. 5, pp. 2537-2552, 1981.

[6] H.P. Freund and A.K. Ganguly, "Electron Orbits in Free-Electron Lasers with Helical Wiggler and Axial Guide Magnetic Fields," IEEE J. Quantum Electron., vol. QE-21, no. 7, pp. 1073-1079, 1985.

[7] D. Prosnitz and E.T. Scharlemann, Lawrence Livermore National Laboratory, ATA Note No. 229, Feb. 22, 1984.

[8] The trajectory code used was developed by K.D. Pendergast, B.G. Danly and M. Tran.

[9] K.R. Chu and A.T. Lin, "Harmonic Gyroresonance of Electrons in Combined Helical Wiggler and Axial Guide Magnetic Fields," Phys. Rev. Lett., vol. 67, no. 23, pp. 3235 - 3238, 1991.

[10] G. Shvets and J.S. Wurtele (private communication). 


\section{FIGURE CAPTIONS}

FIG. 1. Ideal equilibrium electron orbits calculated for two different values of wiggler magnetic field. The solid points show the values of $B_{x}$ where maximum power is observed for Group I, Group II and Reversed Field regimes.

FIG. 2. Free electron laser experimental setup.

FIG. 3. Electron beam current in the FEL as a function of the axial guide magnetic field $B_{z}$; (a) no wiggler magnetic field; (b) wiggler field $\mathrm{B}_{w}=630 \mathrm{G}$ and $\mathrm{B}_{z}$ in the conventional direction; (c) wiggler field $B_{w}=1.47 \mathrm{kG}$ and $B_{z}$ in the reversed direction.

FIG. 4. Angular distribution of rf power emitted by the output conical horn; (a) magnetron only; (b) FEL amplifier in the Reversed Field regime.

FIG. 5. Frequency spectrum of the FEL in the Reversed Field regime, showing five successive shots.

FIG. 6. FEL output power as a function of $B_{z}$ and $B_{w}$; (a) $B_{w} \operatorname{scan}$ for fixed $B_{z}=10.9 \mathrm{kG}$ in each direction; (b) $B_{z}$ scan in the conventional direction for fixed $B_{w}=630 \mathrm{G}$; (c) $B_{z} \operatorname{scan}$ in the reversed direction for fixed $B_{w}=1.47 \mathrm{kG}$.

FIG. 7. FEL output power as a function of interaction length; (a) Group I regime; (b) Group II regime; (c) Reversed Field regime.

FIG. 8. Superradiant operation (without the driver magnetron) of the FEL in the Group I regime; (a) output power as a fuction of $B_{w}$ for fixed $B_{-}=4.06 \mathrm{kG}$; (b) frequency spectrum for many successive shots and for various settings of the spectrum analyzer local oscillator.

FIG. 9. The solid points correspond to averaging over 128 particles values of $v_{x} / c$ calculated numerically by a particle trajectory code. The curves correspond to the ideal equilibrium orbits (see Fig. 1). 


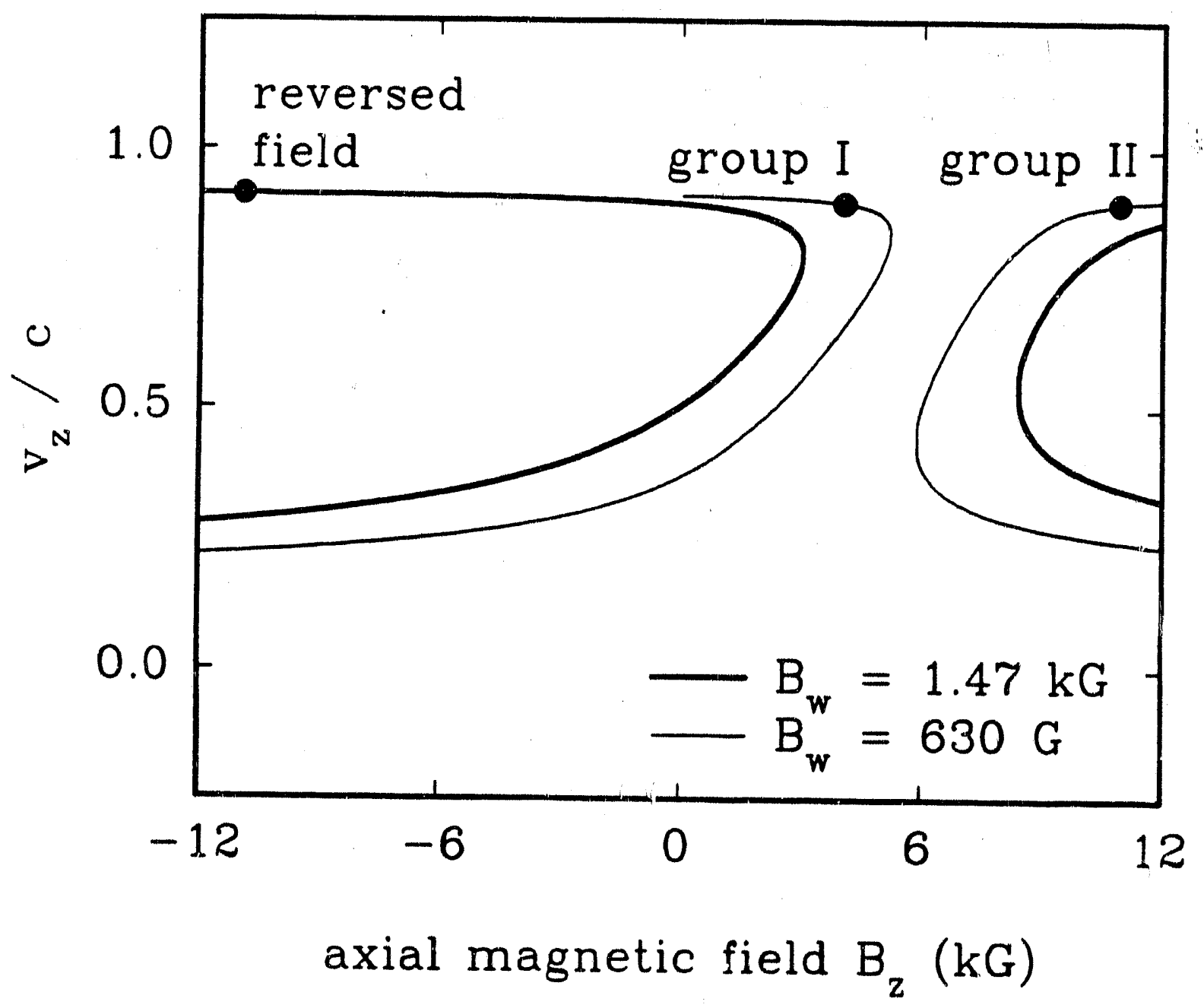




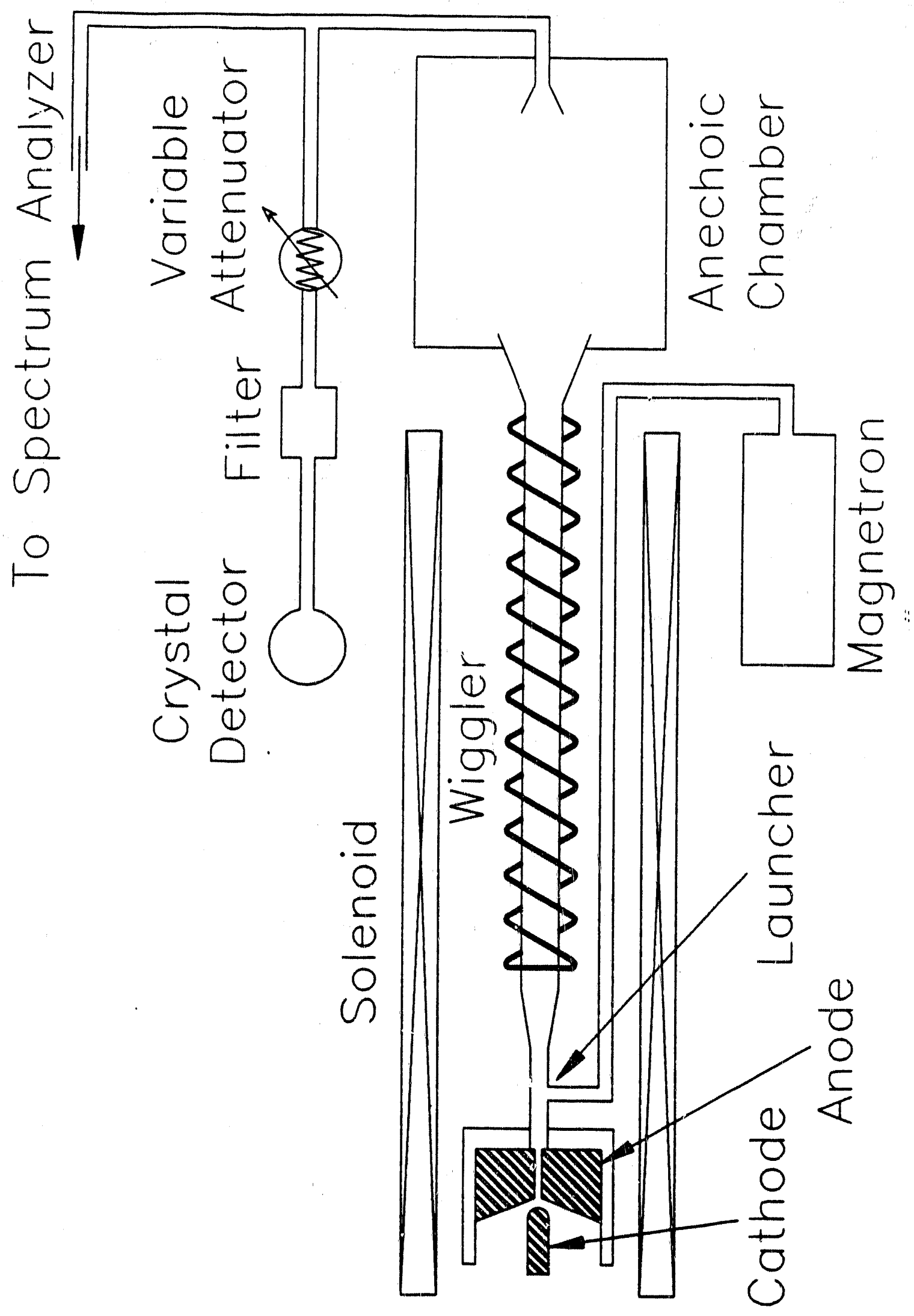



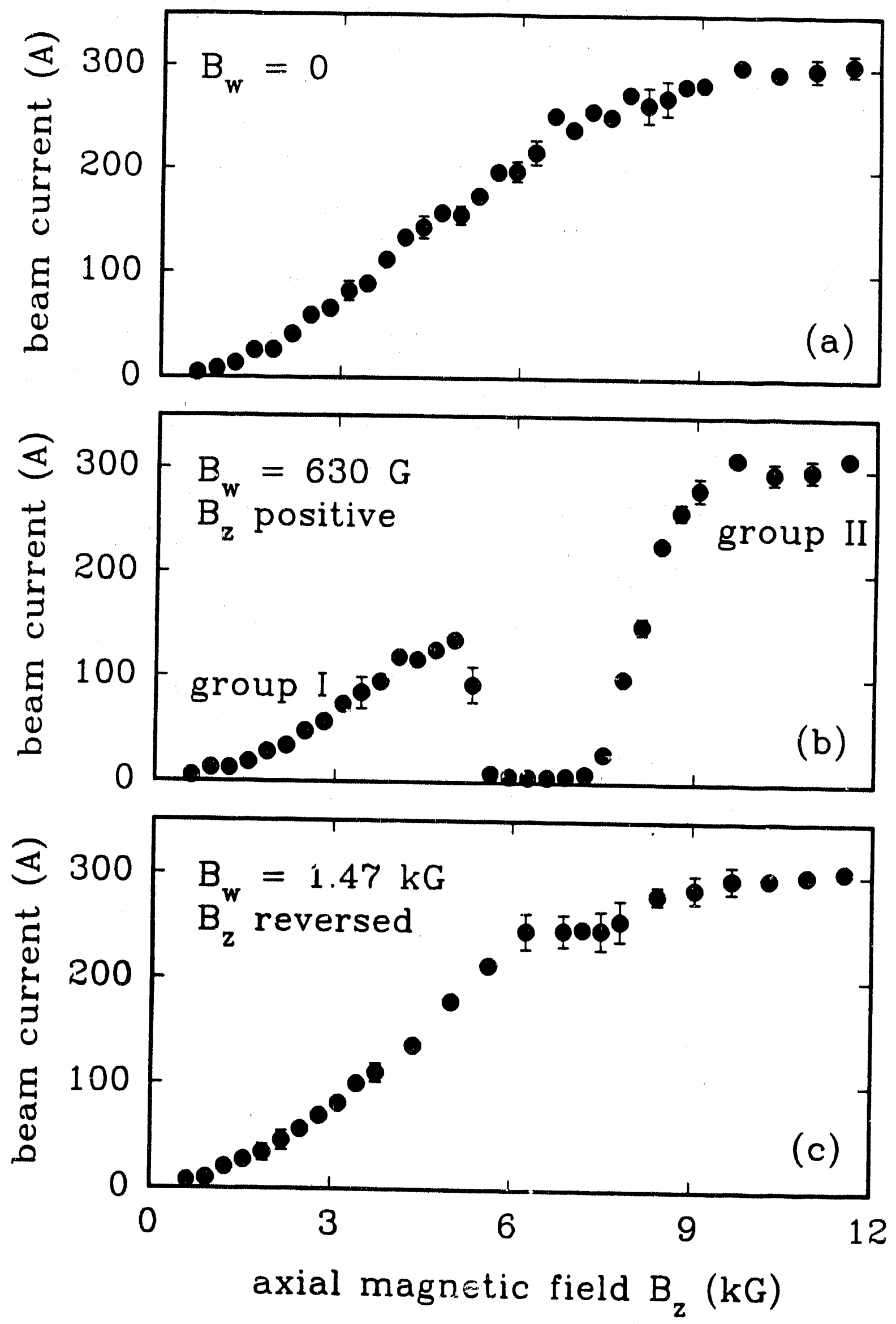

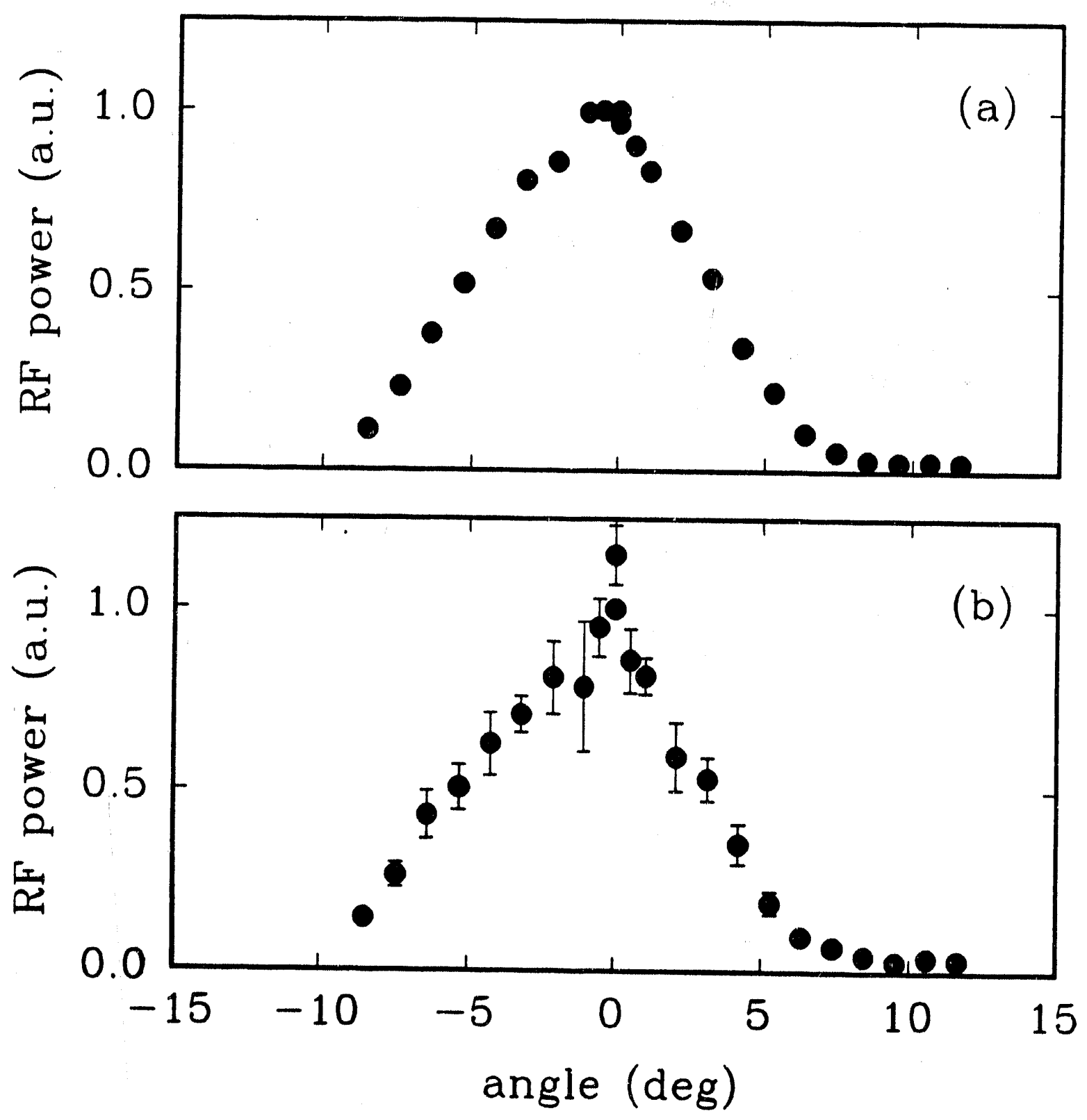


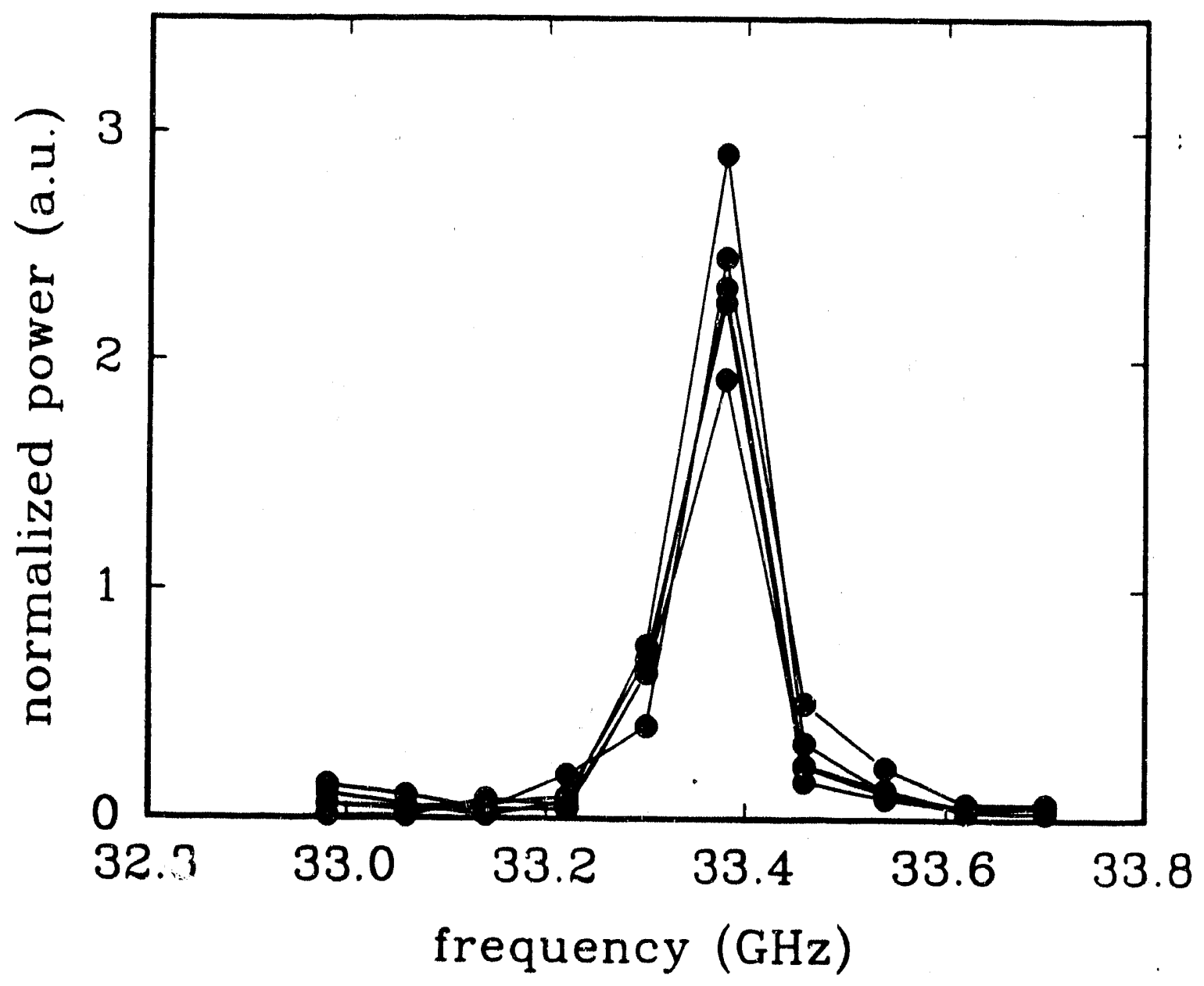



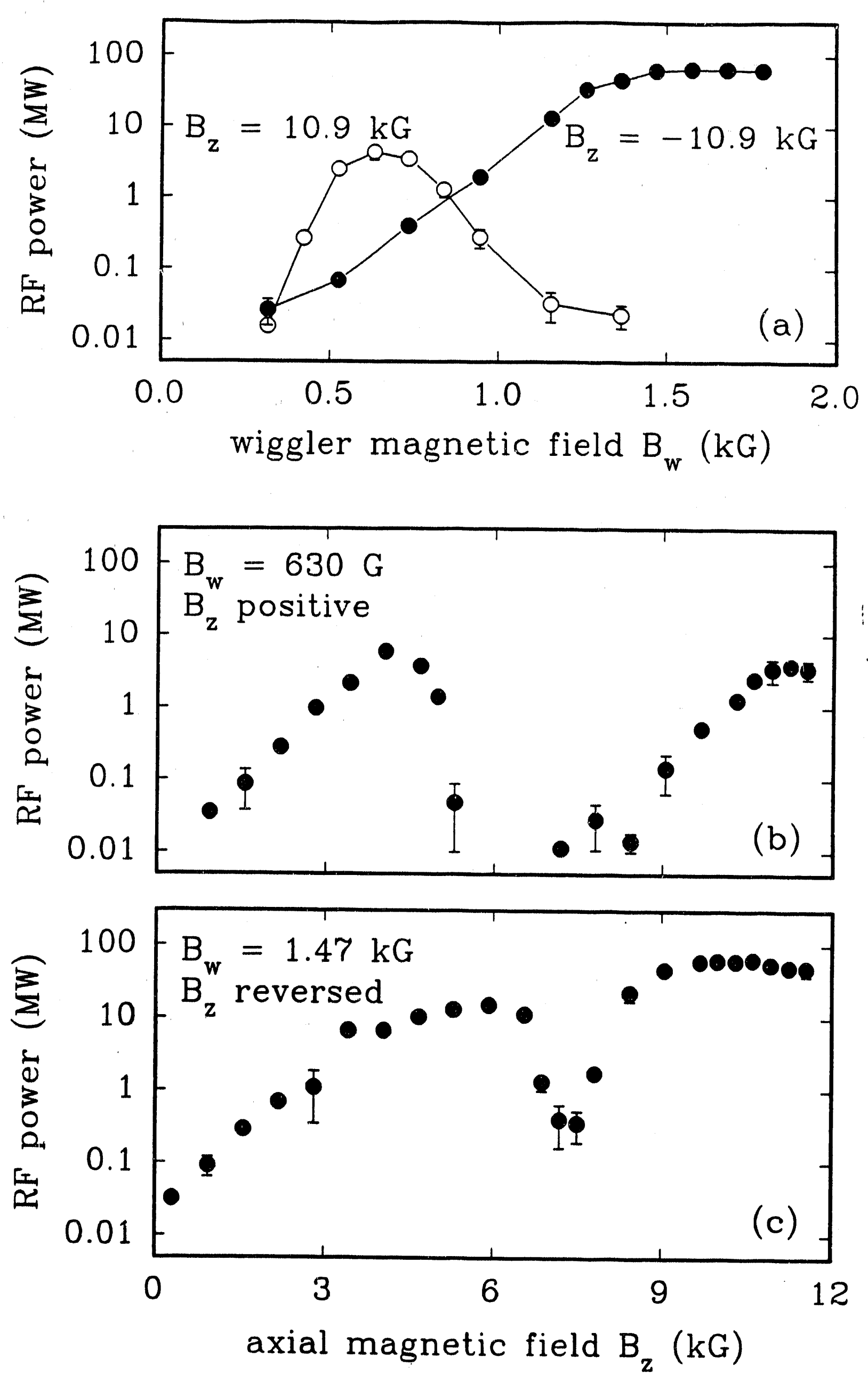

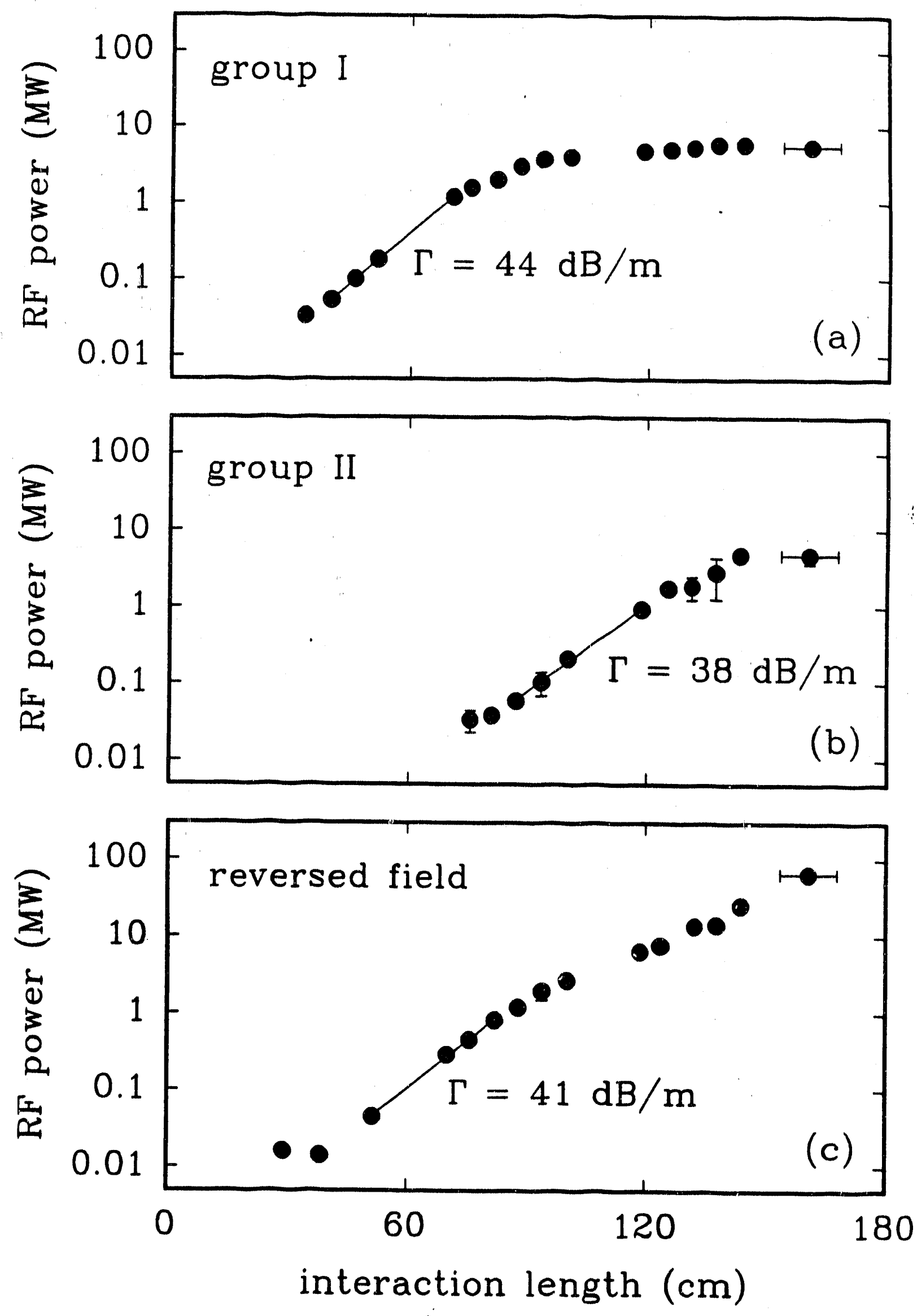

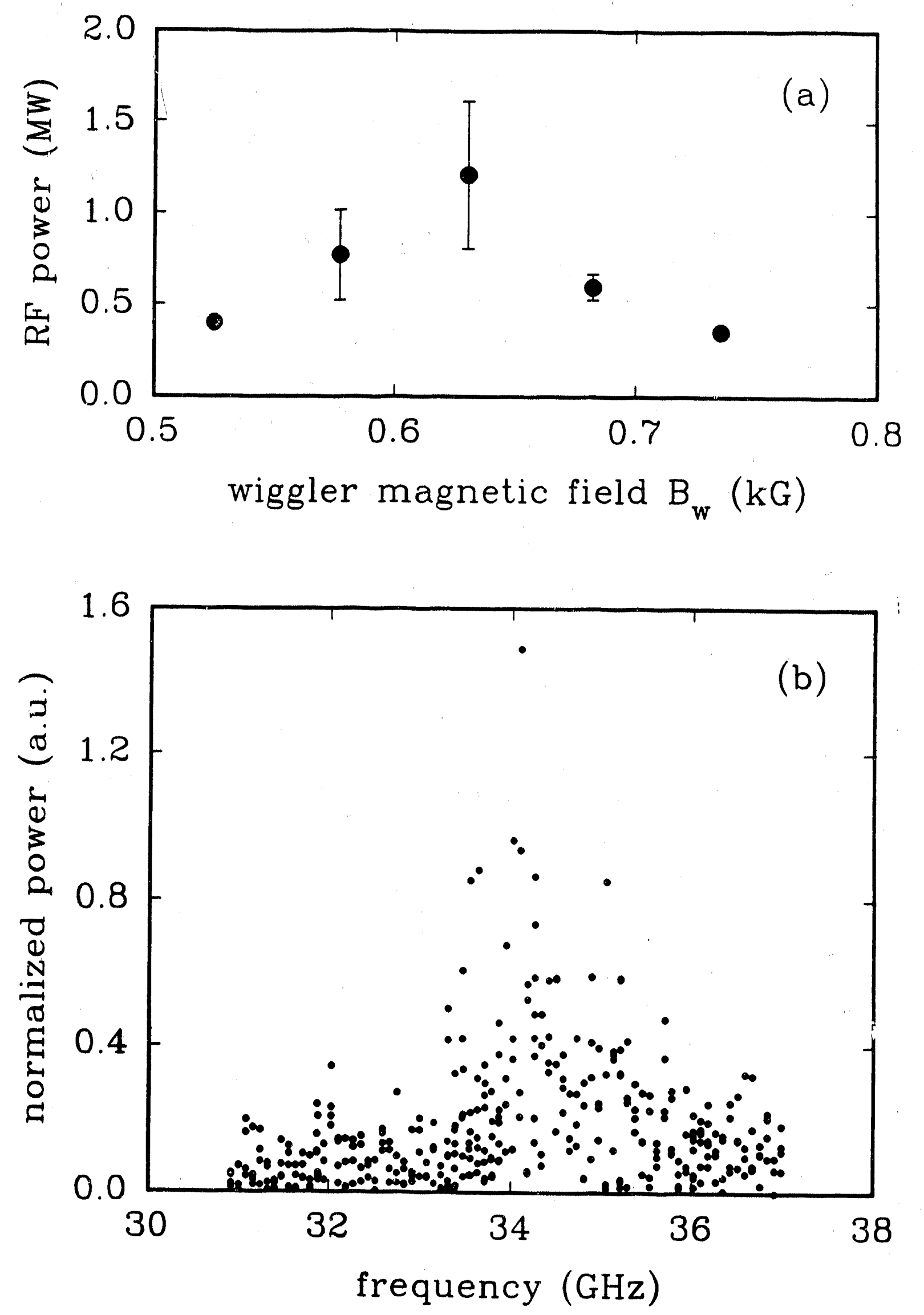


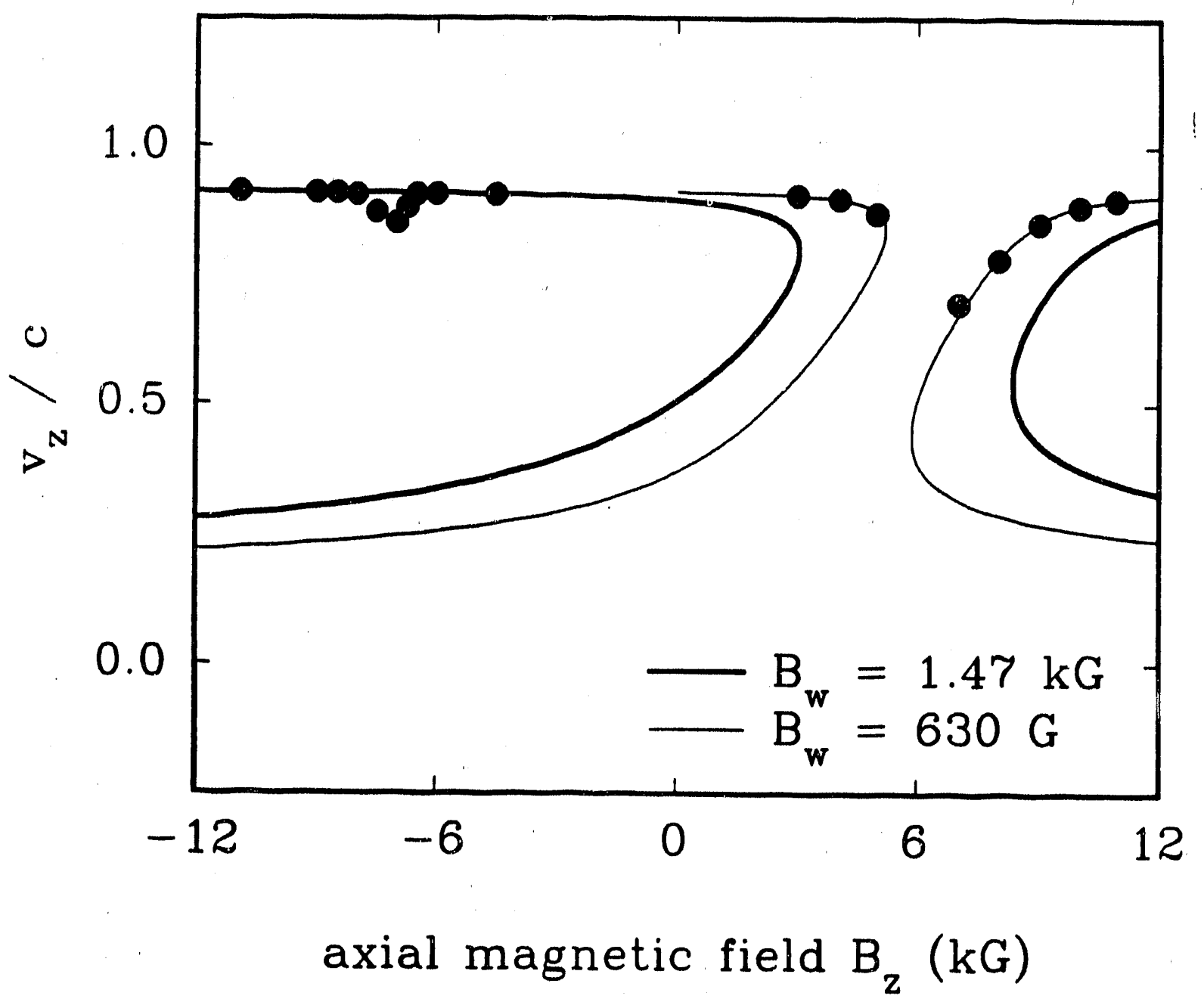


Appendix F. Selected Reprints and Preprints: Theory

removed and cyced separately - 
Appendix G. Publications Supported by This Proposal 


\section{PUBLICATIONS}

Supported all or in part by the Department of Energy

High Energy Physics Division.

1. "Adiabatic Disruption of Asymetric Colliding Beams," T. Katsouleas and J.S. Wurtele, Proc. of ICFA Workshop on Beam Dynamics, Los Angeles, 1991.

2. "Instability Calculations for the MIT-Bates South Hall Ring," K. D. Jacobs, P. T. Demos, J.B. Flanz, A Zolfaghari, J.S. Wurtele, X.T. Yu, and K. Balewski, Proc. of the 1991 Particle Accelerator Conference, IEEE Cat. No. $91 \mathrm{CH} 3038$ -7 , p. 1791 (1992).

3. "Design Modelling of a $17 \mathrm{GHz}$ RF Photocathode Gun," C.L. Lin, S.C. Chen, J.S. Wurtele, R.J. Temkin, and B.G. Danly, Proc. of the 1991 Particle Accelerator Conference, IEEE Cat. No. 91CH3038 -7, p. 2026 (1991).

4. "Phase Stability of a Standing-Wave Free-Electron Laser," W.M. Sharp, G. Rangarajan, A.M. Sessler, and J.S. Wurtele, Proc. SPIE 1407, 535 (1991).

5. A.M. Sessler, D.H. Whittum, J.S. Wurtele, W.M. Sharp, and M.A. Makowski, "A Standing-Wave Free-Electron Laser Two-Beam Accelerator," Nucl. Instr. and Meth. in Phys. Res., (1991)

6. "Scaling Laws for the Cyclotron Resonance Laser Accelerator," C. Chen, Phys. Fluids B3, 2933 (1991).

7. "Effect of Longitudinal Space-Charge Waves of a Helical Relativistic Electron Beam on the Cyclotron Maser Instability," C. Chen, D.G. Danly, G. Sivvets, and J.S. Wurtele, in press, IEEE Trans. Plasma Sci. (1992).

8. "Comparison of Gyro-Averaged And Non-Gyro-Averaged Nonlinear Analyses of Cyclotron Autoresonance Masers," H.P. Freund and C. Chen, Int. J. Electron, in press (1992).

9. "Experimental and Theoretical Study of Periodic Intensity Bursts in the Startup Phase of a Free-Electron Laser Oscillator," E. Jerby, G. Bekefi, and J. S. Wurtele, IEEE J. of Quantum Electronics, QE-27, 2512. (1991).

10. "Design Studies of a 3.3GHz Relativistic Klystron Amplifier," C. Chen, P. Catravas, and G. Bekefi, to be published in Proc. of SPIE Conf. Vol. 1629 (1992).

11. M. E. Conde and G. Bekef, "Experimental Study of a $33.3 \mathrm{GHz}$ Free-Electron Laser Amplifier with a Reversed Axial Guide Magnetic Field," Phys. Rev. Lett $\underline{67}, 3082(1991)$. 
12. "CARM Amplifier Theory and Simulation," C. Chen, B.G. Danly, J.A. Davies. W.L. Menninger, J.S. Wurtele and G. Zhang, Nuclear Instruments and Methods in Physics Research A.304, 593 (1991). 

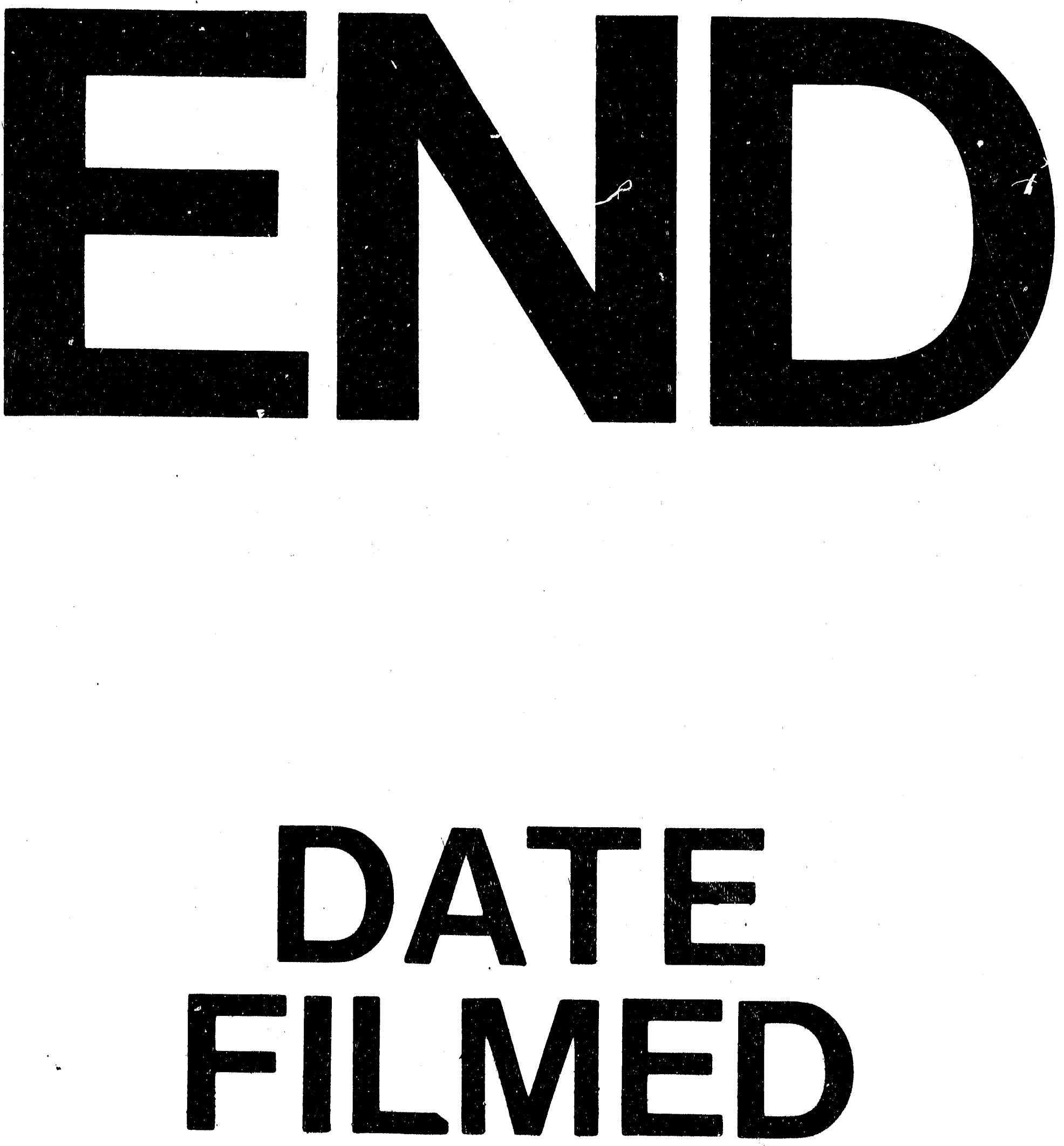

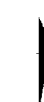

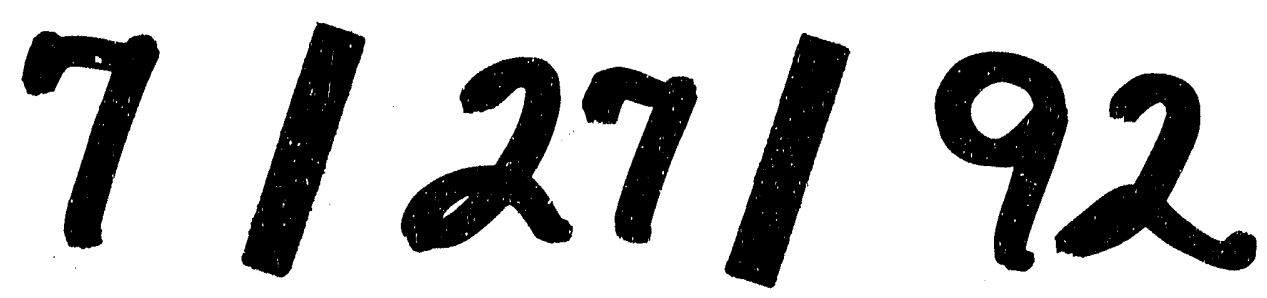


